Universidade de São Paulo

Instituto de Astronomia, Geofísica e Ciências Atmosféricas

Departamento de Astronomia

Bruno Correia Mota

\title{
Um Estudo Teórico da Evolução Temporal das Características Polarimétricas de Estrelas Be
}

\author{
Dissertação de Mestrado \\ Versão Corrigida. O original encontra-se disponível na Unidade.
}

Agosto de 2013 

Bruno Correia Mota

Prof. Dr. Alex Cavaliéri Carciofi

\title{
Um Estudo Teórico da Evolução Temporal das Características Polarimétricas de Estrelas Be
}

\author{
Dissertação de Mestrado \\ Versão Corrigida. O original encontra-se disponível na Unidade.
}

Dissertação apresentada ao Departamento de Astronomia do Instituto de Astronomia, Geofísica e Ciências Atmosféricas da Universidade de São Paulo como requisito parcial para a obtenção do título de Mestre em Ciências.

Área de Concentração: Astronomia Orientador: Prof. Dr. Alex Cavaliéri Carciofi 

à minha Família 



\section{Agradecimentos}

Aos meus pais, por me amarem, mesmo quando não fui o filho que devia ser;

À Karina, pelo seu Amor e por cada vez me fazer querer mais ficar ao seu lado;

Ao Professor Orientador Alex Cavaliéri Carciofi, pela confiança, entusiasmo, bondade, gentileza e por ter apresentando coisas que me transformaram em uma pessoa melhor;

Ao doutorando Daniel Moser Faes, pela amizade, prontidão em sempre ajudar, paciência, discussões e pelas boas horas compartilhadas na F-309 e nas noites frias do OPD;

Aos professores, Augusto Damineli, Antônio Mário Magalhães, Elisabete Dal Pino, Roberto Costa, Ronaldo de Souza, Walter Maciel, pela disposição nas aulas ministradas;

Ao nosso grupo de pesquisa, Allan Pellejero, André Figueiredo, Cyril Escolano, Daiane Breves, Daniel Bednarski, Edgar Ramirez, Jon Bjorkman, Jorge Ribeiro, Leandro Rimulo, Maryory Agudelo, Marcelo Rubinho, Nadili Ribeiro, Rodrigo Vieira, Sérgio Quadros, Xavier Haubois, pelo companheirismo nas missões observacionais, em congressos, pelas discussões em reuniões de grupo e por tornarem o ambiente do IAG tão bom;

Aos amigos e vizinhos de sala, Behrouz Khiali, Fellipy Silva, Luis Kadowaki, Reinaldo Santos-Lima, pelas brincadeiras, discussões, apoio e pelos milhares de abraços sinceros;

Aos amigos, Andrés Páez, Bruno Quint, Felipe Oliveira, Nascimento, Navarete, George, Juan Pineda, Paulo Lago, Silvio Fiorentin, por todos momentos vividos nestes dois anos;

À Ana Carolina Coelho, simplesmente uma página de agradecimentos, como prometido;

Aos funcionários e amigos, Aparecida dos Santos, Carlos Paladini, Marco dos Santos, Marina Freitas, Maria da Conceição, Maria Iacovelli e Ulisses Manzo;

Ao técnico Mauro Januário e ao motorista José Barbosa, por toda ajuda sincera e cuidados durante as noites no OPD-LNA;

Ao INCT-A e à CAPES, pelo apoio financeiro. 
Esta dissertação foi escrita em LATEX com a classe IAGTESE, para teses e dissertações do IAG. 
"Gaio Galo, um amigo de teu pai, Cipião, estudava sem parar o céu e a terra, chegando a calcular quase todas as suas medidas. Quantas vezes foi surpreendido, em pleno dia, completando os desenhos das constelações observadas durante a noite! Quantas vezes foi visto trabalhando em plena noite, quando havia se levantado de manhã cedo! Que prazer ele sentia em nos predizer os eclipses da lua e do Sol!"

Cícero - Saber envelhecer

"Um sábio homem de Agrigento (Empédocles) exprime inclusive em poemas em grego, ao que dizem esta idéia visionária de que, fixas ou móveis, todas as coisas na natureza e em todo o universo se estruturam graças à amizade, e se desconjuntam por causa da discórdia." Cícero - A Amizade 



\section{Resumo}

Estrelas Be são reconhecidas pela sua rápida rotação e pulsação não radial. São as únicas estrelas da Sequência Principal que apresentam discos circunstelares, os quais são formados por meio de processos ainda não completamente compreendidos. A modelagem das forças que atuam neste sistema conduz a previsões teóricas sobre a estrutura do disco que podem ser comparadas com dados observacionais.

Podemos estudar as propriedades físicas dos discos de estrelas Be pelo efeito que a luz estelar sofre ao passar por eles, por exemplo, modelando a transferência radiativa. Neste ponto, a polarização surge como uma ferramenta muito útil para a investigação destes discos, permitindo a determinação de quantidades físicas importantes do sistema, como a densidade numérica de partículas e o ângulo de inclinação.

Uma variabilidade intrigante observada em estrelas Be é a transição aperiódica entre uma fase B normal (sem disco) e uma fase Be (com disco). Estudos de monitoramento recentes encontraram, a partir da análise da polarização intrínseca decorrente da transição entre estas fases, uma relação significante entre a mudança da polarização através do salto de Balmer versus a polarização na banda $V$, fazendo surgir uma estrutura em loop como função do tempo, no assim denominado Diagrama Cor-Polarização.

Neste trabalho, apresentamos uma análise do Diagrama Cor-Polarização por meio de modelos diversos. Fazemos uso do Disco de Decréscimo Viscoso que é o paradigma atual para explicar a formação e evolução dos discos de estrelas Be. Com isso, visamos determinar como a polarimetria pode contribuir para a compreensão dos mecanismos fundamentais envolvidos no processo de formação e dissipação do disco. 



\section{Abstract}

Be stars are recognized by their rapid rotation and non-radial pulsation. They are the only stars in the Main Sequence that have circumstellar disks that are formed by processes not yet fully understood. The modeling of the forces acting on this system leads to theoretical predictions about the structure of the disk that can be compared to observational data.

We can study physical the properties of Be disks by modeling how stellar light is reprocessed by them. This requires solving the detailed radiative transfer problem involved. In this point, the study of polarization arise as a useful tool to investigate these disks, allowing for the determination of important physical quantities of the system, such as the particle number density and inclination angle.

An intriguing variability observed in Be stars is the aperiodic transition between a B normal phase (without disk) to a Be phase (with disk). Recent monitoring studies found, from the analysis of the intrinsic polarization arising of the transition between these phases, a significant relation between the polarization change through the Balmer jump versus the $V$-Band polarization, giving rise to a loop structure as a function of time, in the so-called Color-Polarization Diagram.

This work presents an analysis of the Color-Polarization Diagram by several models. We make use of the Viscous Decretion Disk Model, which assumes the existence of some injection mechanism of material at keplerian velocities in the disk base, where the turbulent viscosity acts carrying angular momentum from de inner parts to the outer regions. With this, we aimed to extend our knowledge about the fundamental mechanisms involved in the formation and dissipation processes of the disk. 



\section{Lista de Figuras}

1.1 Quadrado das visibilidades, ao longo da direção polar (curvas superiores) e equatoriais (curvas inferiores). As linhas tracejadas representam as visibilidades na banda $K$ para discos uniformes com tamanhos angulares de 1.62 mas e 2.53 mas. A linha vertical pontilhada indica a linha de base máxima disponível dos dados do VLTI. As inserções mostram imagens na banda $K$ em escala logarítmica (Carciofi et al., 2008). . . . . . . . . . . . .

1.2 Comparação entre as sequências principais dos modelos com rotação (linha preta sólida) e dos modelos sem rotação de Schaller et al. (1992) (linha azul tracejada) (Ekström et al., 2012). . . . . . . . . . . . . . . . . . 30

1.3 Observações e modelos de $\zeta$ Tauri. Esquerda: SED observada (preto), modelada (azul) e SED da fotosfera estelar (vermelho). Direita: Espectro polarizado observado (preto), modelado (azul), (Carciofi et al., 2009). . . . . . .

1.4 Esquema de uma estrela Be em rotação crítica. A parte inferior mostra como os perfis das linhas espectrais variam indo da posição pole-on para a edge-on (Rivinius e Carciofi, 2013; em preparação). . . . . . . . . . . . .

1.5 SED de $\delta$ Sco para a fase ativa (com disco), os círculos representam as observações e a linha cheia o melhor ajuste. A fase pré-ativa (asteriscos) é mostrada junto com o ajuste. As outras linhas representam os fluxos espalhado, emitido e não processado para a fase ativa (linhas pontilhada, tracejada e ponto-tracejada, respectivamente) (Carciofi et al., 2006). . . . . 34

1.6 Amostragem do disco em diferentes regiões. Nela são mostrados os fluxos em diferentes comprimentos de onda para uma estrela Be típica de subtipo espectral B1Ve (Carciofi, 2011). . . . . . . . . . . . . 
1.7 Perfis H $\alpha$ da estrela $o$ Andromedae medidos entre julho e dezembro de 1986 , mostrando a transição entre uma fase B para uma Be (Clark et al., 2003). . 40

1.8 Curva de luz (painel esquerdo) e diagrama cor-magnitude (painel direito) da estrela OGLE 005209.92-731820.4 (de Wit et al., 2006). . . . . . . . . . 41

1.9 Evolução temporal da polarização intrínseca (painel esquerdo) e o diagrama cor-polarização observado (painel direito) obtido para uma período (pontos vermelhos) (Draper et al., 2011). . . . . . . . . . . . . . .

2.1 Esquema representativo do problema de transporte radiativo. . . . . . . . . 47

2.2 Representação de uma onda elipticamente polarizada vista de frente Ernie Seaquist (2003), Curso de Processos Radiativos. . . . . . . . . . . . . . . 50

2.3 Representação do processo de espalhamento Thomson (Wood et al., 1996b). 53

2.4 Esquema representativo de como ocorre a orientação da polarização num disco de Be, mostra-se o espalhamento a $90^{\circ}$ da luz proveniente de diferentes hemisférios. As componentes paralelas, $I_{\|}$, dos feixes espalhados são, por definição, nulos. O quadro maior em vermelho representa uma ampliação de uma região pequena do disco, junto com os vetores de polarização dos dois feixes considerados. . . . . . . . . . . . . . . . .

2.5 Topo: Espectros polarizados simulados com diferentes valores de fótons, $n_{\mathrm{f}}$, juntamente com os ajustes polinomias (curvas verdes) para uma estrela de subtipo espectral B2 com disco a um ângulo de inclinação de $70^{\circ}$. Abaixo: Gráficos dos resíduos, $R$, dos respectivos ajustes polinomiais. . . . . . . . . 56

2.6 (a) Gráfico da dispersão em função da quantidade de fótons $\left(\sigma \times n_{\mathrm{f}}\right)$ para uma estrela de subtipo espectral B2 com densidade na base $5.0 \times 10^{12} \mathrm{~cm}^{-3}$ e tamanho do envelope de $20 R_{\star}$. A curva vermelha representa o ajuste $\propto \sqrt{n_{\mathrm{f}}}$. 58

3.1 (a) Perfil de temperatura no plano equatorial do disco para os subtipos espectrais de B0 a B4 e (b) a mesma distribuição para o subtipo espectral B2 obtido para três densidades na base do disco. . . . . . . . . . . . . . 
3.2 (a) Distribuição das populações dos níveis de 1 a 3 do hidrogênio ao longo do plano equatorial do disco para os subtipos espectrais B0 e B3. (b) A mesma distribuição para o subtipo espectral B2 com duas densidades na base diferentes. . . . . . . . . . . . . . . . . .

3.3 Profundidade óptica associada às diferentes absortividades, tomada em relação ao comprimento de onda para duas densidades na base do disco (azul: $10^{12}$ partículas por $\mathrm{cm}^{3}$, rosa: $10^{14}$ partículas por $\left.\mathrm{cm}^{3}\right)$. O livre-livre, o espalhamento eletrônico, o ligado-livre e a profundidade total são plotados em pontilhado, tracejado, ponto-tracejada e linha cheia, respectivamente. . . .

3.4 a) Variação radial das absortividades eletrônica e ligado-livre para o subtipo espectral B2 em dois regimes de densidade (vermelho: $1.0 \times 10^{13} \mathrm{~cm}^{-3}$ e azul: $5.0 \times 10^{13} \mathrm{~cm}^{-3}$ ). (b) O mesmo para outros três subtipos espectrais, B0, B2 e B4, para uma mesma densidade na base. São mostrados os ajustes radiais tomados para a região mais externa dos discos. . . . . . . . . . . .

3.5 (a) Nível de polarização e a profundidade óptica associada às diferentes absortividades como função do comprimento de onda ao redor de uma B2 a um ângulo de inclinação de $70^{\circ}$ para um regime de baixa densidade e (b) para um regime de alta densidade (Haubois, Carciofi e Mota (2013), em preparação) . . . . . . . . . . . . . . . . . . . .

3.6 (a) Variação com a escala de densidade do disco da polarização na banda $V, P_{\mathrm{v}}$, e da razão $B J+/ B J-$. (b) Variação com o aumento do raio do disco, da polarização na banda $V, P_{\mathrm{v}}$, e da razão $B J+/ B J-$, para uma dada inclinação e densidade na base. . . . . . . . . . . . . .

3.7 (a) Espectro polarizado de uma estrela B2 tomado para diferentes tamanhos de disco a uma densidade na base de $n_{0}=1.0 \times 10^{13} \mathrm{~cm}^{-3}$. (b) Espectro polarizado de um disco de $20 R_{\star}$ para diferentes subtipos espectrais, tomados para uma densidade na base de $n_{0}=1.0 \times 10^{13} \mathrm{~cm}^{-3} \ldots \ldots \ldots$. . . . . 7

3.8 Nível máximo da polarização no visível de um disco com raio de $20 R_{\star}$ a $70^{\circ}$ versus a temperatura efetiva estelar para três escalas de densidade. . . 72 
3.9 Mudança da polarização na banda $V$ com o ângulo de inclinação do disco, tomada para os subtipos espectrais de B0 a B5. A curva tracejada representa a curva teórica de Brown e McLean (1977) . . . . . . . . . . . . . . .

4.1 (a) Esquema de como simulamos o processo de crescimento do disco variando o tamanho do disco, $r_{\mathrm{d}}$, e (b) o de dissipação do disco, onde consideramos um disco cheio, $r_{\mathrm{d}}=20 R_{\star}$ e buracos internos, $r_{\mathrm{i}}$, progressivamente maiores.

4.2 Fluxo normalizado tomado no contínuo para diversas bandas em diferentes regiões do disco de uma estrela de subtipo espectral B1 com densidade na base de $n_{0}=3.0 \times 10^{13} \mathrm{~cm}^{-3}$.

4.3 (a) Fluxo normalizado tomado no contínuo para diversas bandas em diferentes regiões do disco de uma estrela de subtipo espectral B1 com densidade na base de $n_{0}=1.0 \times 10^{13} \mathrm{~cm}^{-3}$ e (b) o mesmo para a densidade na base de

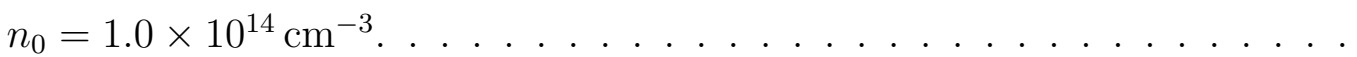

4.4 Diagrama cor-polarização para uma estrela de subtipo espectral B2. Os valores em azul representam os tamanhos do disco na fase de crescimento em unidades de raios estelares. Os valores em vermelho representam os tamanhos de alguns dos buracos internos, em unidades de raios estelares, que simularam a fase de dissipação. . . . . . . . . . . . . . . . .

4.5 (a) Diagrama cor-polarização para cinco subtipos espectrais de B0 a B4. (b) Ampliação da região dos subtipos espectrais mais recentes $(\mathrm{B} 0 \rightarrow \mathrm{B} 2)$. Representamos em dois gráficos devido à diferença entre as escalas apresentadas entre os subtipos espectrais recentes e tardios. . . . . . . . . . .

4.6 Diagrama cor-polarização de uma estrela de subtipo espectral B2 com diferentes densidades de base.

4.7 (a) Diagrama cor-polarização para uma estrela de subtipo espectral B3 tomado para diferentes ângulos de inclinação do disco, $i$. (b) Razão $B J+$ /BJ-tomada para diferentes subtipos espectrais por $i$. . . . . . . . . .

5.1 Curva de luz na banda $V$ da estrela 28 CMa. Os triângulos cinza correspondem às observações e as curvas representam os ajustes feitos para diferentes valores de $\alpha$ (Carciofi et al., 2012). 
5.2 Evolução temporal da densidade superficial durante o crescimento (esquerda) e a dissipação (direita) do disco, para os parâmetros de viscosidade $\alpha=0.1$ e 1. As épocas são contadas em anos e a linha grossa representa o estado estacionário do perfil de densidade. Os pontos pretos indicam o raio externo da região de acreção onde a velocidade radial é nula (ponto de estagnação) (Haubois et al., 2012). . . . . . . . . . . . . . . .

5.3 Curvas de luz na banda $V$ associadas com o crescimento do disco (painel superior) e dissipação (painel inferior). As linhas pontilhadas, tracejadas e ponto-tracejadas representam as curvas de luz para os ângulos de inclinação de $0^{\circ}$ (face-on), $70^{\circ}$ e $90^{\circ}$ (edge-on), respectivamente. As cores preta, vermelha e azul representam os modelos para $\alpha=0.1,0.5$, e 1.0, respectivamente. As linhas sólidas pretas indicam o valor assintótico de $\Delta V$ (Haubois

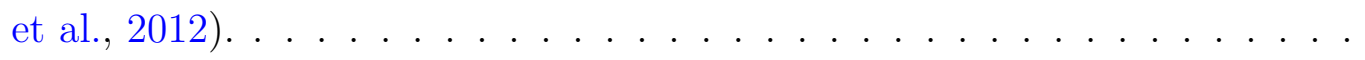

5.4 Evolução temporal de $P_{\mathrm{V}}$ (esquerda) and $P_{\mathrm{BJ}}$ (direita) associadas com o crescimento do disco (painel esquerdo) e dissipação (painel direito). As linhas sólida, pontilhada e tracejada representam as curvas de luz para os ângulos de inclinação de $30^{\circ}, 70^{\circ}$ e $90^{\circ}$, respectivamente. As curvas azuis e vermelhas representam os modelos para $\alpha=0.1$ e 1.0 , respectivamente. As linhas pretas sólidas indicam os valores máximos para cada cenário. . . . .

5.5 DCP em três ângulos de inclinação (linha sólida: $30^{\circ}$, pontilhada: $70^{\circ} \mathrm{e}$ tracejada: $90^{\circ}$ ) para um cenário envolvendo uma fase de crescimento de 100 anos e uma de dissipação de 50 anos para dois parâmetros $\alpha$ (azul: 0.1; vermelho: 1.0). . . . . . . . . . . . . . . . . .

5.6 (a) DCP do $P_{\mathrm{BJ}}$ e $P_{\mathrm{V}}$ para seis subtipos espectrais distintos. A densidade na base é de $6 \times 10^{-11} \mathrm{~g} \mathrm{~cm}^{-3}$ e o ângulo de inclinação é de $85^{\circ}$. (b) DCP do $P_{\mathrm{BJ}}$ e $P_{\mathrm{V}}$ para três densidades: $6 \times 10^{-11} \mathrm{~g} \mathrm{~cm}^{-3}$ (linha sólida), $3 \times 10^{-11} \mathrm{~g} \mathrm{~cm}^{-3}$ (linha tracejada) e $1 \times 10^{-11} \mathrm{~g} \mathrm{~cm}^{-3}$ (linha ponto-tracejada). O cenário dinâmico envolve uma estrela de subtipo espectral B3 com uma fase de crescimento de 50 anos seguida de uma fase de dissipação de 50 anos. O ângulo de inclinação do disco é de $85^{\circ}$. . . . . . . . . . . . . . . 92

B.1 Espectro polarizado de $\zeta$ Tauri Carciofi et al. (2009). . . . . . . . . . . . . 112 
B.2 Diagrama esquemático mostrando a radiação polarizada espalhada por um elemento do disco (linha tracejada) (Bjorkman e Bjorkman, 1994). . . . . . 112

C.1 Superior: (a) O fator de Gaunt livre-livre versus a energia incidente dos elétrons para vários valores de energia de fótons, (b) o fator de Gaunt livrelivre versus a energia dos fótons para vários energias de elétrons incidentes, Karzas e Latter (1961). Inferior: (c) Fator de Gaunt médio ligado-livre sobre uma camada versus a energia de um elétron livre para $n=1 \rightarrow 15$, (d) fator de Gaunt médio ligado-livre versus a energia do fóton para várias energias de elétrons incidentes, (Karzas e Latter, 1961) . . . . . . . . . . . . 117

E.1 Polarização como função da profundidade óptica equatorial para várias inclinações, para um disco com ângulo de abertura de $45^{\circ}$ (Wood et al., 1996b), os círculos maiores são os resultados do espalhamento simples mais atenuação investigados por Fox (1994). . . . . . . . . . . . . . . . . . . 122 


\section{Lista de Tabelas}

3.1 Propriedades médias de estrelas Be de B0 até B5 (Harmanec, 1988). São listadas a temperatura efetiva no polo, $T_{\mathrm{ef}}^{\text {polo }}$, a velocidade crítica, $v_{\text {crit }}$, a luminosidade, $L$, a massa, $M$, o raio equatorial, $R_{\mathrm{eq}}$, e o comprimento de onda em que ocorre o máximo em emissão, $\lambda_{\max } \ldots$. . . . . . . . . . . . . . 61 



\section{Índice}

1. Introdução . . . . . . . . . . . . . . . . . . . . 25

1.1 Resumo do Capítulo . . . . . . . . . . . . . . . . 25

1.2 Apresentação . . . . . . . . . . . . . . . . . . . . 25

1.3 Estrelas de Alta Massa . . . . . . . . . . . . . . . . . . . . 26

1.3.1 Estrelas de Alta Massa e a Rotação Estelar . . . . . . . . . . . . . . 27

1.3.2 Contexto Astrofísico das Estrelas Be . . . . . . . . . . . . . 30

1.4 Discos Circunstelares de Estrelas Be . . . . . . . . . . . . . . . . . . 31

1.4.1 Diagnósticos Observacionais . . . . . . . . . . . . . . . . . . 32

1.4.2 O Modelo de Decréscimo Viscoso . . . . . . . . . . . . . . . 37

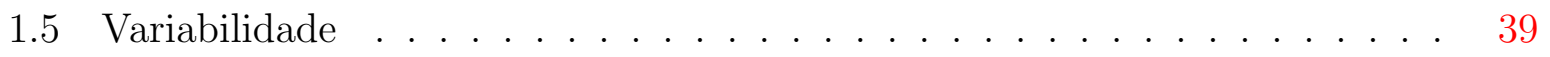

1.5.1 Variações de Longo Período . . . . . . . . . . . . . . . . . . . 39

1.5.2 Diagramas Cor-Polarização . . . . . . . . . . . . . . . . 41

1.6 Organização Geral do Trabalho . . . . . . . . . . . . . . . . . . . . . . . 42

2. Modelos e Ferramentas Teóricas . . . . . . . . . . . . . . . . . . . . 43

2.1 Resumo do Capítulo . . . . . . . . . . . . . . . . . . . . 43

2.2 Processos Radiativos Presentes nos Discos de Be . . . . . . . . . . . . 43

2.2.1 Equilíbrio Radiativo . . . . . . . . . . . . . . . . 44

2.2.2 Processos de Emissão e Absorção . . . . . . . . . . . . . . . . . . 45

2.2 .3 Opacidade . . . . . . . . . . . . . . . . . . . 47

2.2.4 Equação de Transferência Radiativa . . . . . . . . . . . . . . . . 47

2.3 Conceitos Básicos de Polarização . . . . . . . . . . . . . . . . . . . 48 
2.3 .1 Polarização . . . . . . . . . . . . . . . . . . . . . . . . 49

2.3.2 Processos de Polarização da Luz . . . . . . . . . . . . . . . . . 51

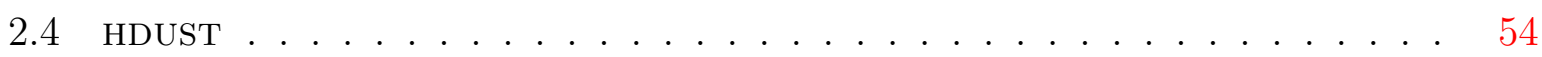

2.4 .1 Determinação dos Erros . . . . . . . . . . . . . . . 55

3. Discos de Estrelas Be em Estado Estacionário . . . . . . . . . . . . . . . . 59

3.1 Resumo do Capítulo . . . . . . . . . . . . . . . . . . . . . . . . . 59

3.2 O Modelo Utilizado . . . . . . . . . . . . . . . . . . . . . . . . . . 59

3.2 .1 Parâmetros Adotados . . . . . . . . . . . . . . . . . . . . 60

3.3 Propriedades Termodinâmicas do Gás . . . . . . . . . . . . . . . . . . . . 61

3.3 .1 A Distribuição de Temperatura . . . . . . . . . . . . . . . . . . 61

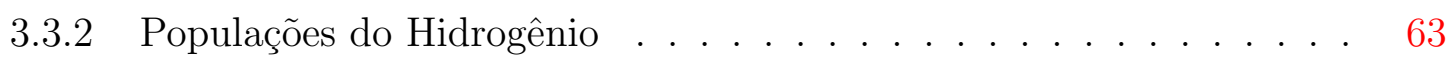

3.4 A Profundidade Óptica . . . . . . . . . . . . . . . . . . . . . . . . . 64

3.4.1 A Profundidade Óptica e a Emissividade . . . . . . . . . . . . . . 64

3.4.2 A Profundidade Óptica Específica para Diferentes Escalas de Densidade . . . . . . . . . . . . . . . . . 65

3.4.3 A Dependência Radial da Absortividade . . . . . . . . . . . . . 66

3.5 Características Polarimétricas de Discos em Estado Estacionário . . . . . . 68

3.5.1 Explicando a Forma do Espectro Polarizado . . . . . . . . . . 68

3.5.2 Dependência do Espectro Polarizado com o Tamanho do Disco e a Escala de Densidade . . . . . . . . . . . . . . . . . 70

3.5.3 Dependência do Espectro Polarizado com o Subtipo Espectral . . 71

3.5.4 Dependência do Espectro Polarizado com o Ângulo de Inclinação do Disco . . . . . . . . . . . . . . . . . . . . 72

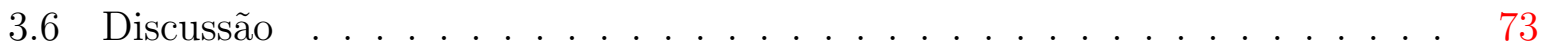

4. Uma Visão do Diagrama Cor-Polarização por meio de Modelos Dinâmicos Simplificados . . . . . . . . . . . . . . . . . . . . . 75

4.1 Resumo do Capítulo . . . . . . . . . . . . . . . . . . . . . 75

4.2 Modelos Dinâmicos Simplificados . . . . . . . . . . . . . . . . 75

4.3 Crescimento e Dissipação . . . . . . . . . . . . . . . . . . . . . . . 77

4.3 .1 Fotometria . . . . . . . . . . . . . . . . 77 
4.3.2 Diagramas Cor-Polarização . . . . . . . . . . . . . . . 77

4.4 Discussão . . . . . . . . . . . . . . . . . . . . . . . . . . . 83

5. O Diagrama Cor-Polarização para o VDD Realista . . . . . . . . . . . . . . 85

5.1 Resumo do Capítulo . . . . . . . . . . . . . . . . 85

5.2 Modelos Dinâmicos Realistas . . . . . . . . . . . . . . 85

5.2 .1 SingleBE . . . . . . . . . . . . . . . 86

5.2.2 A Evolução Temporal da Densidade Superficial . . . . . . . . . . . 87

5.3 Crescimento e Dissipação . . . . . . . . . . . . . . . . . . . 90

5.3 .1 Fotometria . . . . . . . . . . . . . . . 90

5.3.2 Assinaturas Dinâmicas na Polarimetria . . . . . . . . . . . . . . . 90

5.4 Potencial de Diagnóstico . . . . . . . . . . . . . . . . . . . . . 93

5.5 Discussão . . . . . . . . . . . . . . . . . . . . . . . 93

6. Conclusões . . . . . . . . . . . . . . . . . . . . . . . 95

Referências . . . . . . . . . . . . . . . . . . . 97

$\begin{array}{ll}\text { Apêndice } & 105\end{array}$

A. Teoria de Discos Circunstelares . . . . . . . . . . . . . . . . . . 107

A.1 Discos Viscosos Alimentados por Taxas Constantes de Decréscimo . . . . . 107

A.1.1 Perfil Estacionário da Densidade Volumétrica no Equador do Disco 108

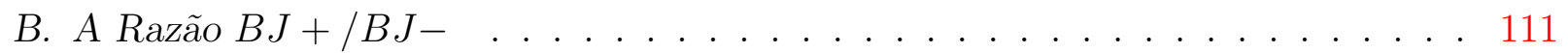

C. Absortividades e Opacidades . . . . . . . . . . . . . . . . . . . . . 113

C.1 Métodos de Determinação das Opacidades . . . . . . . . . . . . . . . . 113

C.2 Dependência Radial das Profundidades Ópticas . . . . . . . . . . . . . . . 114

C.2.1 Profundidade Óptica Eletrônica . . . . . . . . . . . . . . . . . 114

C.2.2 Profundidade Óptica Livre-Livre . . . . . . . . . . . . . . . . . 115

C.2.3 Profundidade Óptica Ligado-Livre . . . . . . . . . . . . . . . . . 115

C.3 Determinação dos fatores de Gaunt . . . . . . . . . . . . . . . . 115 
D. O Salto de Balmer . . . . . . . . . . . . . . . . . . . . . . . . . . 119

E. O Efeito do Espalhamento Múltiplo na Polarização de Envelopes Circunstelares 121

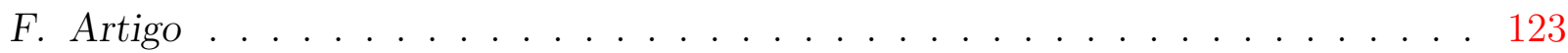


Capítulo 1

\section{Introdução}

\subsection{Resumo do Capítulo}

Esta dissertação trata do estudo da luz polarizada em discos de estrelas Be variáveis, por meio do código HDUST. Neste capítulo, apresentamos, na seção 1.3, a importância do estudo de estrelas de alta massa e de seus envoltórios para a evolução do conhecimento do processo evolutivo destas estrelas e de suas vizinhanças. Na seção 1.3.2, contextualizamos as estrelas Be, mostrando a importância de seu papel no cenário astrofísico e fazemos uma breve introdução sobre seus discos circunstelares na seção 1.4. Terminamos o capítulo apresentando, na seção 1.5, as formas de variabilidade de estrelas Be que constituem uma das principais motivações para nosso trabalho.

\subsection{Apresentação}

Em 23 de Agosto de 1866 o Padre Sechi (1866), diretor do observatório do Collegio Romano, escreveu uma carta ao editor do "Astronomische Nachrichten", reportando

"une particularité de l'étoile $\gamma$ Cassiopée",

que, em vez de uma linha de absorção $\mathrm{H} \alpha$, como observada em Sirius ou Vega, apresentava

"une ligne lumineuse trés belle et bien plus brilliante que tout le reste du spectre".

Esta frase marca a descoberta da primeira estrela Be por meio da análise da linha de emissão H $\alpha$. Curtiss (1916), em seu artigo sobre as linhas de $\gamma$ Cas, argumenta que o estudo destes espectros pode auxiliar na compreensão de casos astrofísicos mais complicados. Mais 
tarde, Struve (1931) presumiu que as linhas deviam se originar de objetos em rotação extremamente rápida; desta forma a rotação faria a estrela se achatar e ejetar matéria através da região equatorial. Posteriormente, Collins (1987) apresentou a definição atual de estrelas Be clássicas, situando-as como estrelas não-supergigantes, ou seja, que estão na Sequência Principal ou saindo dela, cujo espectro apresenta, ou apresentou em algum momento, uma ou mais linhas de Balmer em emissão.

Buscamos, com nosso trabalho, entender os mecanismos físicos presentes em estrelas Be clássicas, assim como em seus discos circunstelares. O estudo da teoria de discos circunstelares tem uma importância astrofísica bastante ampla, muito além do campo das estrelas Be. Por exemplo, ela constitui um importante elemento para a compreensão da formação de estrelas jovens com disco formadas a partir de nuvens em colapso, como as estrelas jovens $\mathrm{T}$ Tauri e as estrelas AeBe de Herbig, que estão na fase de acreção de matéria (Waters e Waelkens, 1998; Williams e Cieza, 2011). Outro exemplo importante são os discos de acreção de buracos negros em centros galácticos (Eckart et al., 2006). Além disso, sistemas sem viscosidade, como os anéis de Saturno, apresentam discos compostos por partículas que não são interagentes entre si e, sob este ponto de vista, são relativamente mais simples do que sistemas circunstelares.

Antes de explorar o tema central desta dissertação, que são as estrelas Be e seus discos, faremos uma breve revisão sobre a importância astrofísica das estrelas de alta massa e de como as estrelas Be se encaixam neste contexto.

\subsection{Estrelas de Alta Massa}

Estrelas de alta massa $\left(M \gtrsim 5.0 M_{\odot}\right)$ influenciam profundamente seu meio em diferentes escalas espaciais, sendo progenitoras dos fenômenos mais energéticos do Universo, tais como supernovas e surtos de raios gama, GRB $^{1}$, tornam-se as grandes responsáveis pela criação de regiões H II e pelo enriquecimento do meio interestelar com elementos pesados processados em seus núcleos. Além disso, seus ventos são responsáveis por introduzir grandes quantidades de energia mecânica no meio circundante. Pelo fato de possuírem alta massa, estas estrelas apresentam rápida evolução na Sequência Principal, podendo, por isso, serem utilizadas como traçadores da formação estelar recente nas galáxias.

\footnotetext{
${ }^{1}$ Gamma-Ray Burst.
} 
Até recentemente, a formação de estrelas massivas era pouco compreendida, mas modelos têm lançado luz a esta questão. Por exemplo, Krumholz (2012) mostra como resultados observacionais atuais têm fornecido vínculos adicionais que podem auxiliar na construção de modelos de formação de estrelas massivas. Estes modelos devem levar em consideração os problemas de fragmentação, binaridade e de pressão de radiação, de forma a explicar resultados observacionais, como o da predileção de estrelas de alta massa por sistemas múltiplos. Devem, além disso, gerar funções de massa estelar inicial, $\mathrm{IMF}^{2}, d N / d M \propto M^{-\Gamma}$, com $\Gamma \approx 2.3$ (Bastian et al., 2010), compatíveis com as observações.

A evolução de estrelas massivas após a Sequência Principal tem se tornado melhor compreendida. Em um estudo recente (Groh et al., 2013) foram analisados modelos de evolução para estrelas em rotação com massas entre $20 M_{\odot}$ e $25 M_{\odot}$ prestes a explodirem como supernovas (e.g. Fig. ??). Os resultados apresentados sugerem que estrelas instáveis conhecidas como LBV ${ }^{3}$ seriam possíveis progenitoras de supernovas de tipo II, o que antes era exclusividade de estrelas supergigantes vermelhas.

As propriedades estelares, tais como a velocidade de rotação, a metalicidade e a presença de campo magnético, podem alterar o esquema evolutivo de estrelas massivas proporcionando resultados reveladores. Dentre estes parâmetros destaca-se, nos modelos de evolução estelar, a taxa de perda de massa. Para estrelas massivas, ela pode exercer papel preponderante, pois a perda de massa destas estrelas pode ser de muitas massas solares no decorrer da vida na Sequência Principal, chegando a cerca de $90 \%$ de sua massa inicial em toda a vida da estrela.

\subsubsection{Estrelas de Alta Massa e a Rotação Estelar}

Estudos mostraram que a rotação estelar pode influenciar de várias maneiras o esquema evolutivo de estrelas de alta massa (e.g. Meynet e Maeder, 2000). A primeira é que a rotação possui profundo impacto na evolução estelar. Por exemplo, Meynet e Maeder (2000) argumentam que após a fase na Sequência Principal, os efeitos da contração do núcleo e a expansão do envelope dominam a evolução do momento angular. Além disso, a alta taxa de perda de massa destas estrelas pode fazer as velocidades superficiais diminuírem durante a

\footnotetext{
${ }^{2}$ Initial Mass Function.

${ }^{3}$ Luminous Blue Variable Star.
} 
evolução na Sequência Principal, gerando uma remoção significativa de momento angular. Um resultado interessante é que estrelas com massas abaixo de $\sim 12 M_{\odot}$ com velocidade de rotação inicial elevada podem atingir a velocidade crítica ${ }^{4}$ próximo do fim de sua estadia na Sequência Principal, o que pode explicar a ocorrência de estrelas Be (Meynet e Maeder, 2000). Outros efeitos importantes originam-se da rotação estelar, a saber:

1. A deformação geométrica ou achatamento dos polos gerado pela alta rotação estelar. O efeito foi detectado diretamente através de medições interferométricas da estrela Achernar (Domiciano de Souza et al., 2003; Carciofi et al., 2008) e da estrela Altair (Monnier et al., 2007).

2. Efeito de escurecimento gravitacional ou de von Zeipel para estrelas quentes (von Zeipel, 1924): este efeito é caracterizado pela aumento do fluxo emitido dos polos para o equador como resultado da baixa gravidade efetiva $\left(T_{e f} \propto g^{0.25}\right)$, já tendo sido observado através da interferometria (e.g. Domiciano de Souza et al., 2012).

Parte da evolução recente no campo de estudo de estrelas Be se deve ao emprego da interferometria. Um exemplo da contribuição dada pela interferometria é o trabalho de Carciofi et al. (2008), que apresenta como o disco residual da estrela Achernar, constatado a partir de uma pequena emissão $\mathrm{H} \alpha$ (Vinicius et al., 2006), pode alterar o sinal interferométrico e quais efeitos isto pode ter na determinação de parâmetros fundamentais como o achatamento e a rotação estelar. Foi verificado que a presença de um disco residual torna-se uma possibilidade real para se explicar as medidas interferométricas. O principal resultado obtido pelos autores está no fato de que esta estrela deve estar girando a uma velocidade muito próxima da crítica, uma vez que somente os modelos com $\Omega / \Omega_{\text {crit }}>0.992$ conseguiram reproduzir as medidas interferométricas e o perfil da linha $\mathrm{H} \alpha$.

A figura 1.1 mostra os resultados de dois modelos de alta rotação que reproduzem bem as observações. O modelo 2, painel esquerdo, idealiza um disco denso em equilíbrio hidrostático, ou seja, um disco geometricamente fino. O modelo 3, painel direito, representa um disco menor e menos denso com uma escala de altura aumentada, ou seja, um disco geometricamente espesso.

\footnotetext{
${ }^{4} \mathrm{~A}$ velocidade crítica, $v_{\text {crit }}$, é a velocidade na qual a força centrífuga balanceia a força gravitacional no equador, tal que a gravidade efetiva se anule.
} 

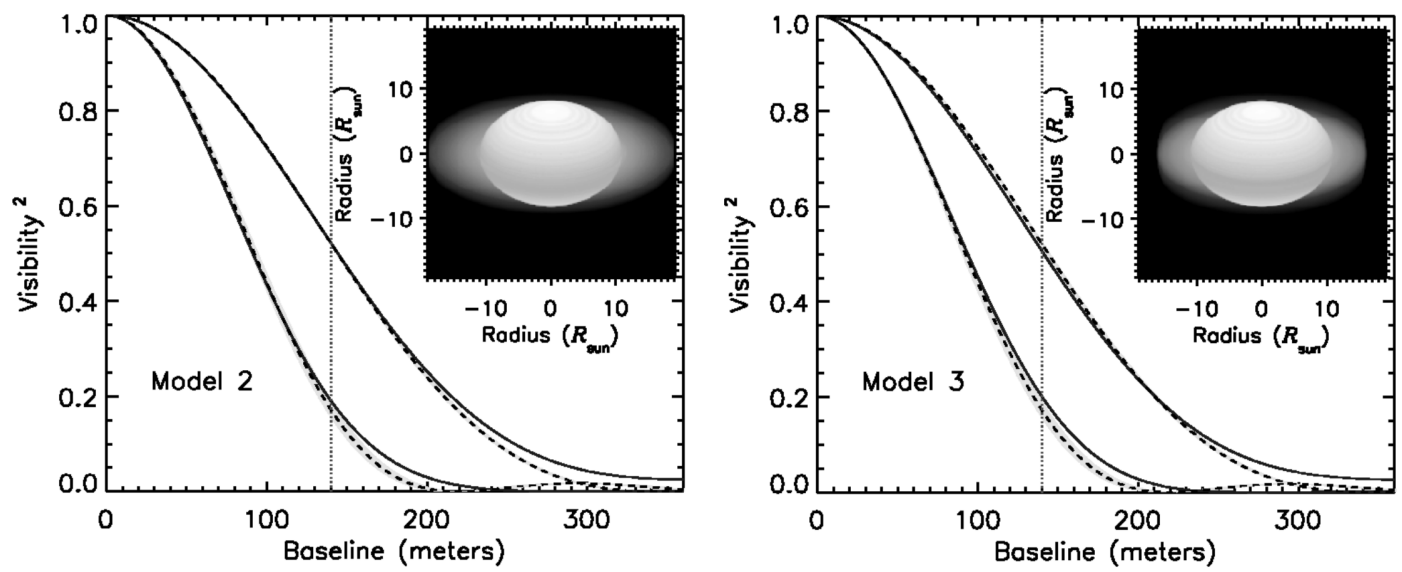

Figura 1.1: Quadrado das visibilidades, ao longo da direção polar (curvas superiores) e equatoriais (curvas inferiores). As linhas tracejadas representam as visibilidades na banda $K$ para discos uniformes com tamanhos angulares de 1.62 mas e 2.53 mas. A linha vertical pontilhada indica a linha de base máxima disponível dos dados do VLTI. As inserções mostram imagens na banda $K$ em escala logarítmica (Carciofi et al., 2008).

Apesar do recente progresso neste campo, a rotação de estrelas Be é ainda um enigma (Cranmer, 2005). Ainda há grande dificuldade na determinação da taxa de rotação através da espectroscopia, a qual apresenta resultados muito incertos. Por exemplo, Cranmer (2005) e Townsend et al. (2004) mostram que a taxa de rotação pode estar bem mais próxima da velocidade crítica do que se imaginava, quando é levado em consideração o efeito de escurecimento gravitacional na análise dos dados. Desta forma, o fluxo correspondente às regiões de alta velocidade torna-se reduzido, ou seja, as regiões de menor velocidade acabam tendo um peso maior no espectro total. Isto faz com que as velocidades projetadas, $v \sin i$, calculadas sem o escurecimento gravitacional, sejam subestimadas, principalmente para valores $v_{\text {eq }} / v_{\text {crit }} \gtrsim 0.8$, onde $v_{\text {eq }}$ é a velocidade equatorial. Este ponto marca um limite superior, a partir do qual não há variação importante no perfil de linha fotosférico.

Como já estipulado por Struve e por outros, a rotação é parte essencial do fenômeno Be. Por exemplo, no caso de uma estrela girando criticamente o material estará em órbita kepleriana no equador e quanto menor a velocidade de rotação maior será a energia necessária para o material se desprender da estrela. Por exemplo, para uma velocidade de rotação de $v_{\text {eq }} / v_{\text {crit }} \simeq 0.7$, é necessário um incremento na velocidade de $100 \mathrm{~km} \mathrm{~s}^{-1}$ para por o material em órbita; porém, para $v_{\text {eq }} / v_{\text {crit }} \simeq 0.95$ o incremento é de apenas $10 \mathrm{~km} \mathrm{~s}^{-1}$. 


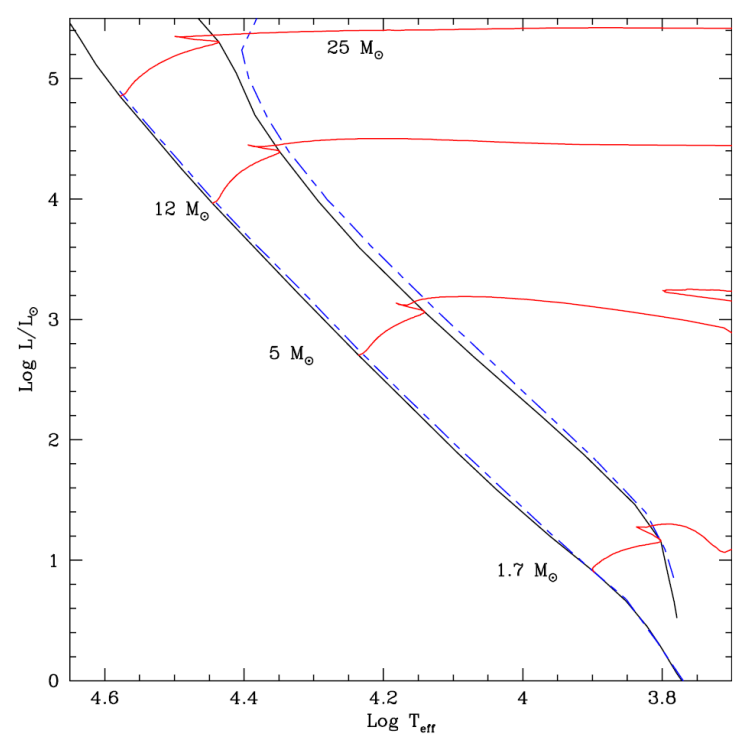

Figura 1.2: Comparação entre as sequências principais dos modelos com rotação (linha preta sólida) e dos modelos sem rotação de Schaller et al. (1992) (linha azul tracejada) (Ekström et al., 2012).

\subsubsection{Contexto Astrofísico das Estrelas Be}

Resultados de trabalhos recentes sobre as trajetórias evolutivas de estrelas de alta massa (Ekström et al., 2012), que apresentam modelos evolutivos de estrelas quentes com rotação comparados com modelos sem rotação (ver Fig. 1.2), demonstram que há uma diferença evolutiva não desprezível.

Para entendermos melhor o papel da rotação em estrelas Be devemos ter em mente que estrelas muito massivas (B e O) giram muito rápido, mas não tanto quanto estrelas Be. Estas últimas são extremos que apresentam velocidades suficientemente elevadas para interferir em suas estruturas internas e, portanto, modificar sua evolução. Por este motivo, se torna imprescindível estudá-las a fim de se obter mais indícios que apontem sobre aspectos que possam detalhar o ciclo de vida destes sistemas.

Hoje, acredita-se que a rotação aumenta durante o envelhecimento da estrela na Sequência Principal, podendo ser um resultado proveniente da contração do núcleo estelar que, por sua vez, geraria o aumento da rotação das partes externas devido à conservação do momento angular (e.g. Meynet e Maeder, 2000). 

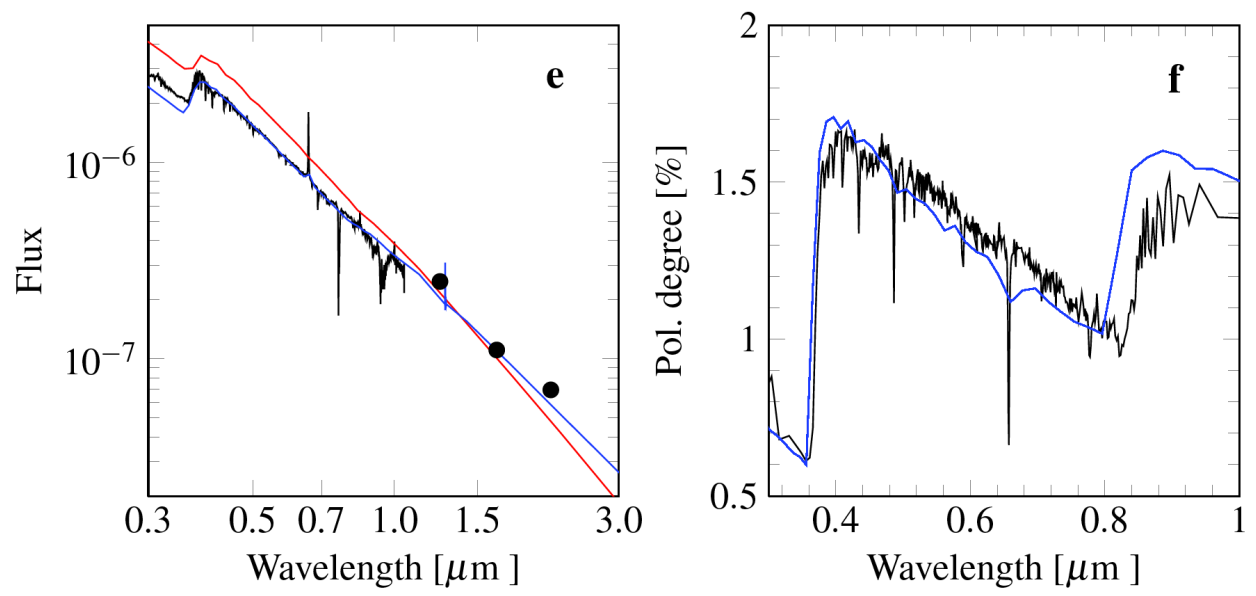

Figura 1.3: Observações e modelos de $\zeta$ Tauri. Esquerda: SED observada (preto), modelada (azul) e SED da fotosfera estelar (vermelho). Direita: Espectro polarizado observado (preto), modelado (azul), (Carciofi et al., 2009).

\subsection{Discos Circunstelares de Estrelas Be}

Nossa concepção sobre as propriedades do material circunstelar em torno de estrelas Be evoluiu muito desde os trabalhos pioneiros de Struve (1931), que foi o primeiro a propor um modelo viável para explicar as linhas de emissão presentes no espectro destas estrelas. O leitor interessado encontrará uma revisão histórica da pesquisa em Be's em Underhill e Doazan (1982), Slettebak (1988) e Porter e Rivinius (2003).

$\mathrm{Na}$ última década, vimos surgir um consenso de como estes discos são estruturados graças a avanços tanto do ponto de vista observacional quanto teórico. Vimos o paradigma do disco de decréscimo viscoso, $\mathrm{VDD}^{5}$, se fortalecer apoiado tanto pelas observações quanto pela teoria, tendo nosso grupo no IAG fornecido contribuições interessantes na parte de transporte radiativo e de teoria de discos circunstelares com o auxílio do código Monte Carlo de transporte radiativo, o HDust. Por exemplo, Carciofi et al. (2009) apresentaram o primeiro teste realmente crítico do VDD mostrado na figura 1.3. Nesta figura, mostra-se o ajuste da SED ${ }^{6}$ e do espectro polarizado de $\zeta$ Tauri.

Modelar discos de Be é uma tarefa complexa, pois o gás circunstelar está ionizado e as baixas densidades $\left(\rho<10^{-10} \mathrm{~g} \mathrm{~cm}^{-3}\right)$ implicam num equilíbrio termodinâmico não-local, $\mathrm{NLTE}^{7}$. Resolver o problema de transferência radiativa em um regime NLTE em geometrias

\footnotetext{
${ }^{5}$ Viscous Decretion Disk, vide apêndice A.

${ }^{6}$ Spectral Energy Distribution.

${ }^{7}$ Non-Local Thermodynamic Equilibrium, ver seção 2.2 .
} 
2D e 3D é notoriamente complexo tendo evoluído significativamente apenas recentemente (Carciofi e Bjorkman, 2006). Por outro lado, a estrutura do disco, que é definida radialmente pela viscosidade e verticalmente pelo equilíbrio hidrostático, é fortemente dependente da temperatura (Carciofi e Bjorkman, 2008). Recentes progressos sugerem que o material do disco é transportado para fora sob influência de torques viscosos ainda não muito bem compreendidos.

Sob o ponto de vista observacional, sabemos que estrelas Be são marcadas por possuírem discos circunstelares que podem ser detectados via linhas espectrais em emissão sobre o espectro da fotosfera, pelo excesso de emissão no infravermelho, IV, resultante do reprocessamento da luz estelar pelo disco, e pela polarização da luz estelar pelo disco (seção 1.4.1). Mais recentemente, deixamos de estudar o disco indiretamente, passando a estudá-lo diretamente através de medidas de alta resolução angular permitidas pela interferometria (seção 1.4.1). Além disso, estrelas Be apresentam variabilidade que pode se manifestar em vários observáveis, como nos perfis de linha de emissão e na polarização linear, abrangendo variadas escalas de tempo que podem ir de décadas a minutos. De forma geral, acredita-se que as variações de longo período estejam relacionadas com mudanças nas propriedades globais do disco circunstelar, enquanto que as variações de curto período estariam ligadas às alterações na fotosfera estelar ou na parte mais interna do disco. Discutimos mais detalhadamente os aspectos das variabilidades na seção 1.5.

Nas duas subseções que seguem, fazemos uma revisão mais detalhada dos diagnósticos observacionais disponíveis para os discos de Be e da teoria de decréscimo viscoso.

\subsubsection{Diagnósticos Observacionais}

Existem diferentes processos físicos operando no disco, cada um associado a diferentes observáveis; desta forma, a diversificação das observações pode gerar resultados complementares, afunilando a física necessária para compreender as estrelas Be e dando sustentação ao processo de modelagem. Neste ponto, um dos principais métodos que sustentam nosso trabalho é a polarimetria.

A seguir, discutimos os resultados observacionais mais relevantes para nosso estudo. 

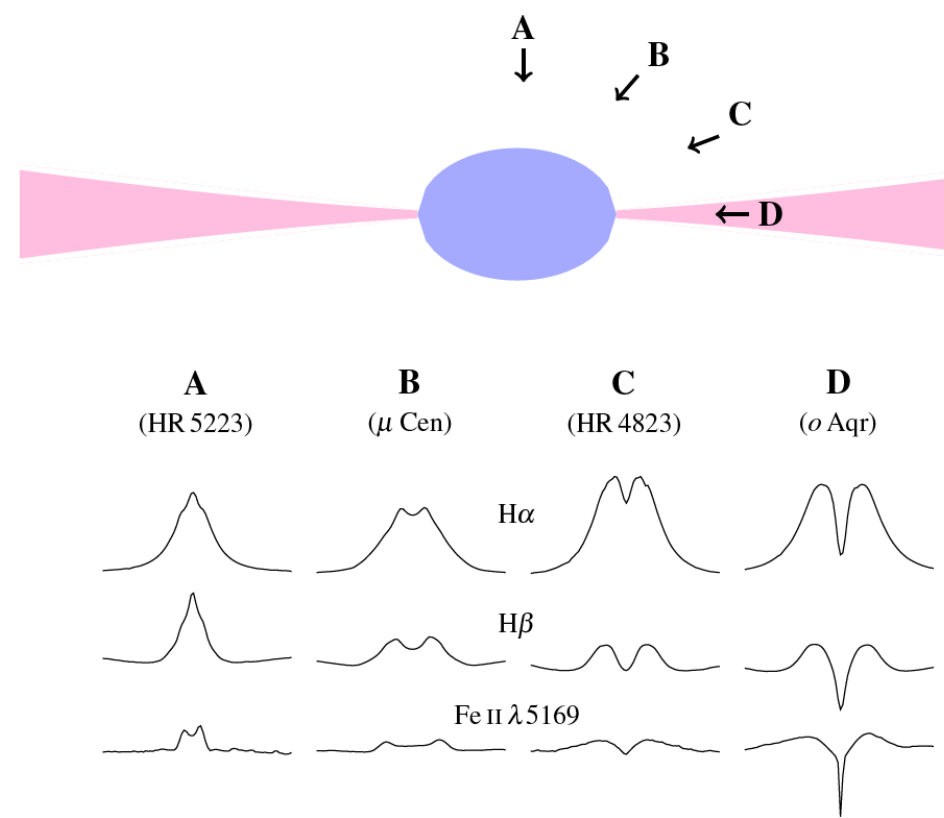

Figura 1.4: Esquema de uma estrela Be em rotação crítica. A parte inferior mostra como os perfis das linhas espectrais variam indo da posição pole-on para a edge-on (Rivinius e Carciofi, 2013; em preparação).

\section{Espectroscopia}

A razão pela qual os espectros de Be apresentam excesso no contínuo ou linhas de emissão está no fato do denso material do disco atuar como uma "pseudofotosfera" muito maior que a fotosfera estelar (Carciofi, 2011). Uma característica importante, já prevista por Struve (1931), é a dependência das linhas com o ângulo de inclinação do disco (ver Fig. 1.4).

Uma linha-diagnóstico muito importante é a H $\alpha$, correspondente à transição dos elétrons que possuem número quântico principal $n=3$ para o $n=2$ no átomo de hidrogênio $(6563 \AA)^{8}$. Outras linhas de emissão importante são as do Fe II que podem ser utilizadas como traçadoras do campo de velocidade do disco (Hummel e Hanuschik, 1997), por apresentarem o perfil característico de duplo pico para diferentes inclinações do disco. Apresentamos, na figura 1.4, um exemplo dos perfis de emissão de diferentes linhas para quatro estrelas Be. Temos, a partir da direita, a emissão em duplo pico característica de discos vistos a ângulos de inclinação próximos de $90^{\circ}$. Por exemplo, o perfil da linha do Fe II 5169 é um exemplo clássico de perfil de linha shell, em que se tem uma forte compo-

\footnotetext{
${ }^{8}$ Num átomo de hidrogênio os níveis são degenerados, ou seja, dois ou mais diferentes estados físicos podem apresentar o mesmo nível de energia.
} 


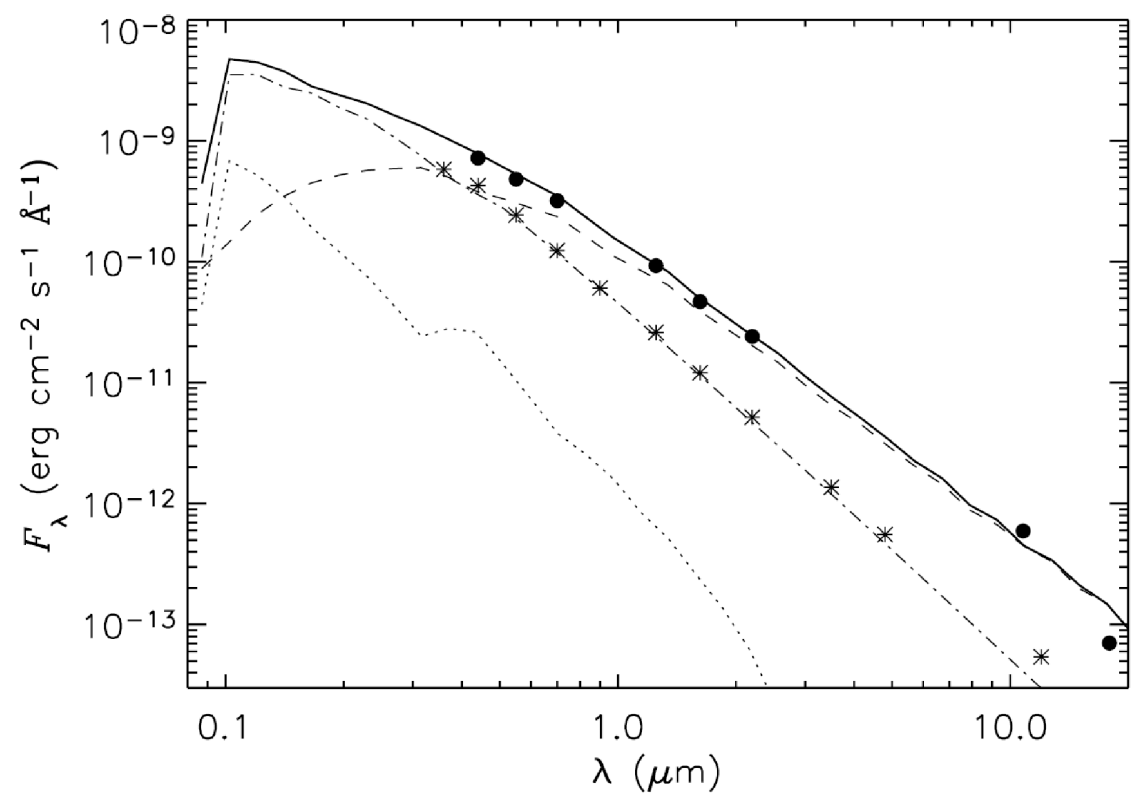

Figura 1.5: SED de $\delta$ Sco para a fase ativa (com disco), os círculos representam as observações e a linha cheia o melhor ajuste. A fase pré-ativa (asteriscos) é mostrada junto com o ajuste. As outras linhas representam os fluxos espalhado, emitido e não processado para a fase ativa (linhas pontilhada, tracejada e ponto-tracejada, respectivamente) (Carciofi et al., 2006).

nente de absorção central, devido ao disco estar entre o observador e a estrela. Isto ocorre pois estrelas shell são estrelas Be vistas de lado. Agora, prosseguindo para a esquerda da figura, nos aproximamos de discos vistos a ângulos de inclinação cada vez mais próximos de $0^{\circ}$. Nestes casos, o perfil de duplo pico desaparece gradualmente, por causa da pouca velocidade projetada do disco, fazendo o perfil tornar-se basicamente gaussiano.

\section{Fotometria}

Partindo agora para o espectro no contínuo, sabemos que a diferença entre o espectro da estrela e do disco está no fato deles terem diferentes temperaturas e densidades. O espectro emitido pelo disco será tipicamente mais vermelho, pelo fato do disco ser mais frio, levando ao conhecido excesso de IV. Fisicamente, parte da radiação no ultravioleta, que é onde ocorre o pico de emissão da estrela, é absorvida e reprocessada em comprimentos de onda maiores, gerando o excesso. A figura 1.5 mostra a distribuição de energia espectral (SED) de $\delta$ Scorpi. Os pontos em asterisco apresentam a estrela antes de possuir o disco, enquanto que a curva cheia apresenta a fase com o disco. 


\section{Espectro-interferometria}

Quirrenbach et al. (1997), utilizando dados interferométricos, derivaram um limite superior, $20^{\circ}$, para o ângulo de abertura do disco de $\zeta$ Tauri e outras estrelas. Em outro estudo Wood et al. (1997) mostraram, por meio da espectropolarimetria, que a geometria do ambiente circunstelar é melhor explicada pela presença de um disco geometricamente fino ou um disco geometricamente espesso com ângulos de abertura de $2.5^{\circ}$ e $52^{\circ}$, respectivamente. A conclusão deste trabalho foi que a combinação da interferometria com a espectroscopia permitiu determinar de forma inequívoca que o entorno circunstelar de estrelas Be é uma estrutura geometricamente fina, o que mostra o grande potencial de diagnóstico proveniente da combinação da interferometria com a polarimetria. Desde então, as concepções de que o material circunstelar seria um elipsóide achatado caíram em desuso (Porter e Rivinius, 2003).

Recentemente, a espectro-interferometria foi capaz de investigar o campo de velocidades do disco, mostrando que ele gira de forma kepleriana. O interesse principal em se determinar uma lei de rotação para o disco está no fato da rotação do disco estar ligada ao seu mecanismo de formação. Pode-se vislumbrar três possibilidades de velocidade para o disco (Carciofi, 2011); a de um disco dominado por campos magnéticos, no qual a componente azimutal da velocidade do disco seria $v_{\phi} \propto r$ (rotação rígida), a de um disco dominado pela pressão de radiação, onde $v_{\phi} \propto r^{-1}$ (conservação do momento angular) e a de um dirigido pela viscosidade cinemática do gás, com $v_{\phi} \propto r^{-1 / 2}$.

Mais recentemente, com o auxílio da espectro-interferometria e espectroastrometria de alta-resolução pôde-se provar diretamente que a dinâmica de discos Be (Carciofi, 2011; Oudmaijer et al., 2011; Štefl et al., 2011) deve ser muito próxima à kepleriana. Este é um importante resultado, o qual posiciona a viscosidade como o mecanismo de condução do outflow.

\section{Espectropolarimetria}

Outra técnica com grande potencial de diagnóstico é a espectropolarimetria, que fornece informações complementares através tanto do nível de polarização quanto da inclinação do espectro de polarização. 
Para entender o processo de polarização da luz, devemos relembrar que antes da radiação proveniente da estrela encontrar o disco ela ainda não está polarizada ${ }^{9}$; isto significa que uma estrela sem disco deve apresentar polarização nula ou desprezível. O ingrediente básico para que ocorra a polarização da luz pelo disco circunstelar é a existência de elétrons livres que por meio do espalhamento Thomson geram a polarização (seção 2.3). Desta forma, como veremos, o nível de polarização está fortemente relacionado com o subtipo espectral da estrela, com a escala de densidade e com o ângulo de inclinação do disco. Além disso, outro aspecto curioso chama a atenção na estrutura do espectro polarizado de discos de Be (Fig. 1.3): seu aspecto serrilhado. Veremos adiante, nos capítulos 2 e 3, porque isto acontece, mas podemos adiantar que esta aparência está relacionada com a presença de hidrogênio neutro no disco.

\section{Amostragem de Regiões Distintas do Disco}

Carciofi (2011) apresentou um estudo sobre a amostragem de diferentes regiões do disco quando observado em diferentes comprimentos de onda. Na figura 1.6, foi calculado o espectro emergente de estrelas Be com discos de diferentes raios externos com o objetivo de se determinar em quais comprimentos de onda as diferentes regiões do disco contribuem mais para o fluxo. No painel esquerdo, a intersecção de diferentes linhas com a linha horizontal tracejada marca a posição no disco de onde cerca de $95 \%$ do excesso de fluxo provém. Por exemplo, vemos que o excesso na banda $V$ é formado muito perto da estrela, dentro de cerca de $2 R_{\star}$, enquanto que o excesso em $1.0 \mathrm{~mm}$ origina-se de uma área muito maior do disco.

O painel direito apresenta como a linha de emissão e a polarização no contínuo crescem com o raio. Para as linhas opticamente espessas de $\mathrm{H} \alpha$ e $\mathrm{Br}-\gamma$, a emissão do disco apenas preenche o perfil de absorção fotosférica quando o tamanho do disco é de aproximadamente $5 R_{\star}$. Ambas linhas possuem pseudofotosferas que se estendem até cerca de $20 R_{\star}$.

Para a polarização, os resultados estão em desacordo com a crença comum de que a polarização é formada próxima da estrela. Notamos que $95 \%$ da máxima polarização na banda $V$ é atingida apenas quando o tamanho do disco é de cerca de $10 R_{\star}$. A figura 1.6 mostra que a polarização linear surge tipicamente da escala de 1 a $10 R_{\star}$.

\footnotetext{
${ }^{9} \mathrm{Na}$ verdade, a luz fotosférica é linearmente polarizada, porém apresentam níveis desprezíveis.
} 

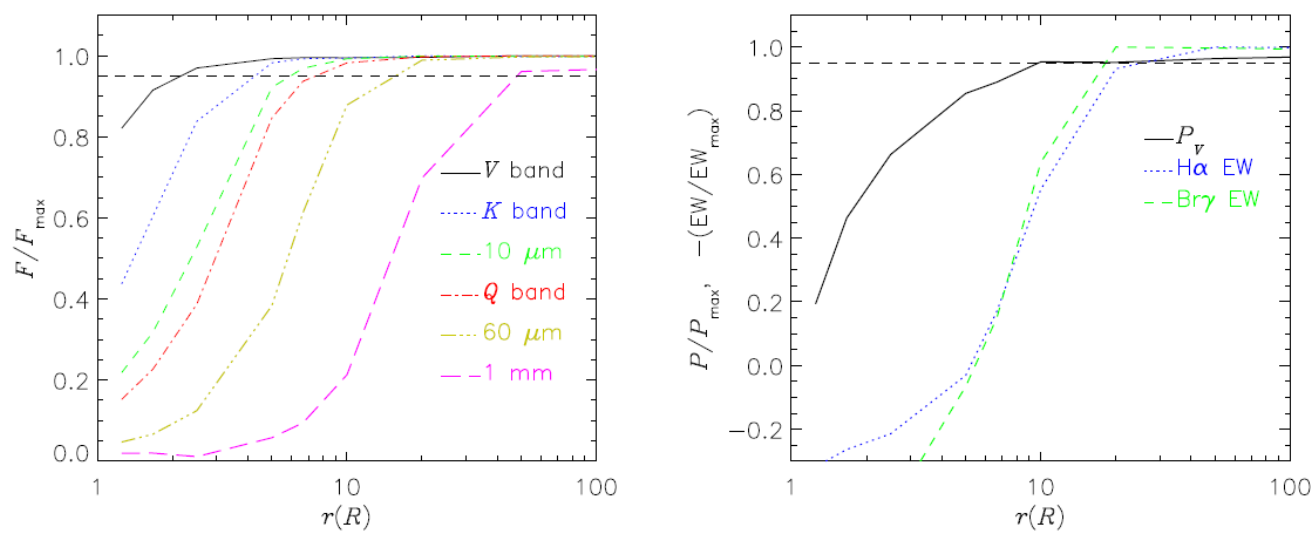

Figura 1.6: Amostragem do disco em diferentes regiões. Nela são mostrados os fluxos em diferentes comprimentos de onda para uma estrela Be típica de subtipo espectral B1Ve (Carciofi, 2011).

Em resumo, se quisermos estudar regiões mais internas do disco o observável que devemos adotar, como a análise nos aponta, é a fotometria em comprimentos de onda curtos. Já as partes mais externas do disco devem ser amostradas pelas linhas de emissão do H I e também pela fotometria no infravermelho. A polarização, ao contrário da fotometria e espectroscopia, é uma medida capaz de amostrar as duas regiões do disco. Apresentamos, na seção 3.5, a causa desta bivalência, demonstrando esta proposição por meio da análise da variação do nível de polarização para diferentes tamanhos de disco.

\subsubsection{O Modelo de Decréscimo Viscoso}

Nas seções precedentes, vimos quais são os diagnósticos observacionais usualmente empregados para se estudar os discos de estrelas Be. As observações fornecem-nos importantes informações estruturais sobre o disco, que devem ser necessariamente reproduzidas por qualquer teoria que se proponha a descrever como estes discos se formam. Por exemplo, a polarimetria e a interferometria (Quirrenbach et al., 1997, Wood et al., 1997) determinaram que os discos são geometricamente finos, enquanto que a espectro-interferometria determinou que o material do disco gira em torno da estrela de forma kepleriana. Até o momento, a única teoria capaz de satisfazer os vínculos observacionais é o modelo de disco de decréscimo viscoso proposto por Lee (1991) e desenvolvido por vários outros autores (Porter, 1999, Okazaki, 2001, Bjorkman e Carciofi, 2005).

Este modelo é o mesmo empregado para discos protoestelares (Pringle, 1981), a diferença primária sendo que discos de Be são outflowing, enquanto discos da pré-sequência princi- 
pal são inflowing. No VDD, é suposto que algum mecanismo, ainda desconhecido, injeta material numa velocidade orbital kepleriana na base do disco. Neste ponto, a viscosidade turbulenta começa a atuar, fornecendo momento angular ao material e transportando-o das regiões internas para fora do disco.

Está fora do escopo desta dissertação uma descrição aprofundada do VDD. Entretanto, dada a importância deste modelo para nosso estudo, apresentamos uma breve descrição das suas propriedades básicas no apêndice A. Neste apêndice, partindo das hipóteses simplificadoras de uma taxa constante de perda de massa e disco isotérmico, calculamos o perfil estacionário de densidade volumétrica no plano médio do disco, $z=0$ (apêndice A.1.1). Neste caso, o disco apresenta um perfil de densidade na forma de uma lei de potência apresentando um expoente radial $n=3.5$,

$$
\rho(r)=\rho_{0}\left(\frac{R_{0}}{r}\right)^{3.5},
$$

onde $\rho$ é a densidade de massa do gás e $R_{0}$ está associado ao tamanho do disco.

Outra expressão importante (apêndice A.1.1) é a da escala de altura do disco,

$$
H(r)=\frac{c_{\mathrm{s}}}{v_{\text {crit }}\left(R_{0}\right)^{1 / 2}} r^{3 / 2}
$$

onde $v_{\mathrm{c}}$ é a velocidade crítica,

$$
v_{\text {crit }}=\sqrt{\frac{G M_{\star}}{R_{\text {eq }} \equiv R_{\star}}}=\sqrt{\frac{2 G M_{\star}}{3 R_{\text {polo }}}},
$$

e $c_{\mathrm{s}}=(k T)^{1 / 2}\left(\mu m_{\mathrm{H}}\right)^{-1 / 2}$ é a velocidade do som no meio. Nesta última expressão, $k$ é a constante de Boltzmann, $\mu$ é o peso molecular do gás e $m_{\mathrm{H}}$ é a massa do átomo de hidrogênio.

É interessante analisar a equação 1.1 do seu aspecto funcional para entender como funciona a variação radial do material que compõe um disco de Be. Esta equação implica numa rápida diminuição da densidade conforme nos afastamos da região interna do disco e, como veremos no capítulo 3, isto influencia fortemente o comportamento das diferentes opacidades no disco. Entretanto, devemos levar em consideração que o valor de $n$ pode sofrer alterações se forem incluídos outros efeitos físicos como o de um processo de difusão viscosa não isotérmica (Carciofi e Bjorkman, 2008). Por sua vez, a equação 1.2 mostra que 
a escala de altura é $H \propto r^{1.5}$. Para exemplificar, calculemos a largura da escala de altura prevista para uma estrela Be típica (ver tabela 3.1; pág. 61). Por exemplo, para uma B0 temos uma escala de altura fornecida pela expressão $H(r) \simeq 0.02\left(r^{3} / R_{0}\right)^{0.5}$, ou seja, para a região próxima da estrela a escala de altura é de apenas $0.02 R_{\star}$, gerando um disco fino como determinado pela interferometria.

\subsection{Variabilidade}

Os tipos de variabilidade presentes em estrelas Be podem ser tanto periódicas quanto aperiódicas, podendo ocorrer em várias escalas de tempo. Variabilidades de curto período, por exemplo, provavelmente estão relacionadas a efeitos magnéticos na fotosfera ou na parte mais interna do disco e não serão tratadas nesta dissertação; a um leitor interessado indicamos o trabalho de Rivinius et al. (2006). Outro tipo de variabilidade são as cíclicas, conhecidas como $V / R$ e relacionadas a ondas de densidade no disco. Isso já foi estudado por outros autores (Kato, 1983; Okazaki, 1991; Okazaki, 1997; Carciofi et al., 2009) e também não fará parte desta dissertação. O terceiro tipo são variações aperiódicas de longo período associadas ao processo de formação do disco. Variações deste tipo associam-se ao processo de evolução secular dos discos de Be e constituem o tema central da dissertação.

\subsubsection{Variações de Longo Período}

Um dos mais intrigantes tipos de variabilidade observadas em estrelas Be é a transição aperiódica entre uma fase B normal (sem disco) e uma fase Be (com disco) (Wisniewski et al., 2010). Nesta transição, o disco é perdido e reconstruído em escalas de tempo de meses a anos (Carciofi, 2011). Em termos observacionais esta variação manifesta-se através de mudanças nos perfis de linha (Clark et al., 2003), no brilho do contínuo, na cor (Harmanec, 1983) e na polarização (Draper et al., 2011). A figura 1.7 apresenta um exemplo de tal transição para a estrela o And (Clark et al., 2003). Em 26 de Agosto de 1986, por exemplo, o perfil de linha era puramente fotosférico, indicando a ausência de disco. Nas semanas que se seguiram, ocorreu a formação de um disco, evidenciado tanto pela emissão nas asas da linha quando pela forte absorção central. Esta absorção central mostra que o disco está sendo visto de lado.

O processo de criação e dissipação do disco pode ser estudado através da fotometria. 


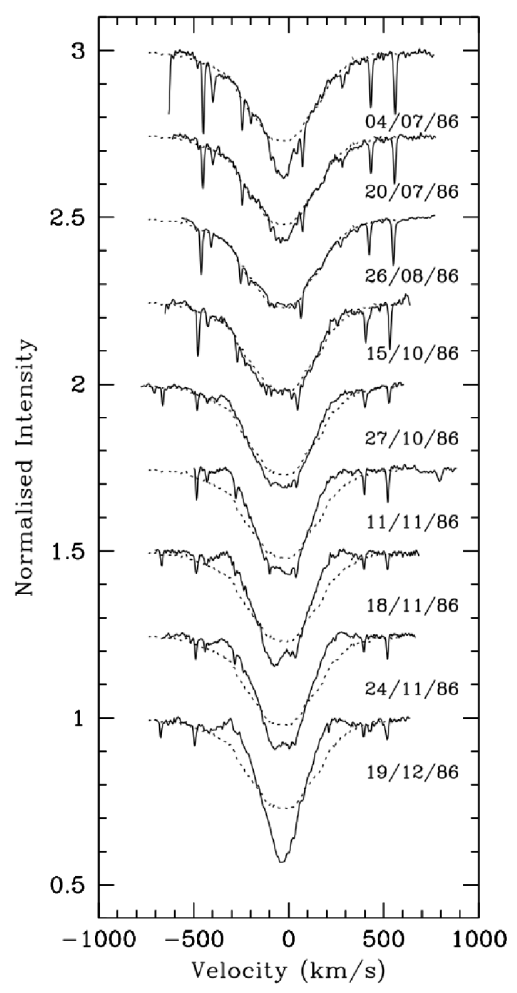

Figura 1.7: Perfis $\mathrm{H} \alpha$ da estrela $o$ Andromedae medidos entre julho e dezembro de 1986, mostrando a transição entre uma fase B para uma Be (Clark et al., 2003).

Um exemplo de curva de luz de uma estrela variável é mostrado na figura 1.8. Para entender o que ocorre neste sistema, de Wit et al. (2006) dividem a curva de luz em 4 fases principais que representam a transição $\mathrm{B} \rightarrow \mathrm{Be} \rightarrow \mathrm{B}$ para a estrela OGLE 005209.92-731820.4 da Pequena Nuvem de Magalhães. Estas fases são:

- Fase I: Marcada pelo rápido aumento do fluxo tanto no azul quanto no vermelho, correspondente ao início do crescimento do disco;

- Fase II: Representa o período de saturação, em que a variação temporal do fluxo se torna mais lenta;

- Fase III: Apresenta o período de reversão associado ao início da dissipação do disco, no qual os fluxos no azul e no vermelho começam a diminuir, com o fluxo no azul prevalecendo durante um tempo maior;

- Fase IV: Progressão da dissipação, fazendo o espectro global (estrela + disco) ser cada vez mais dominado pelo espectro estelar. 


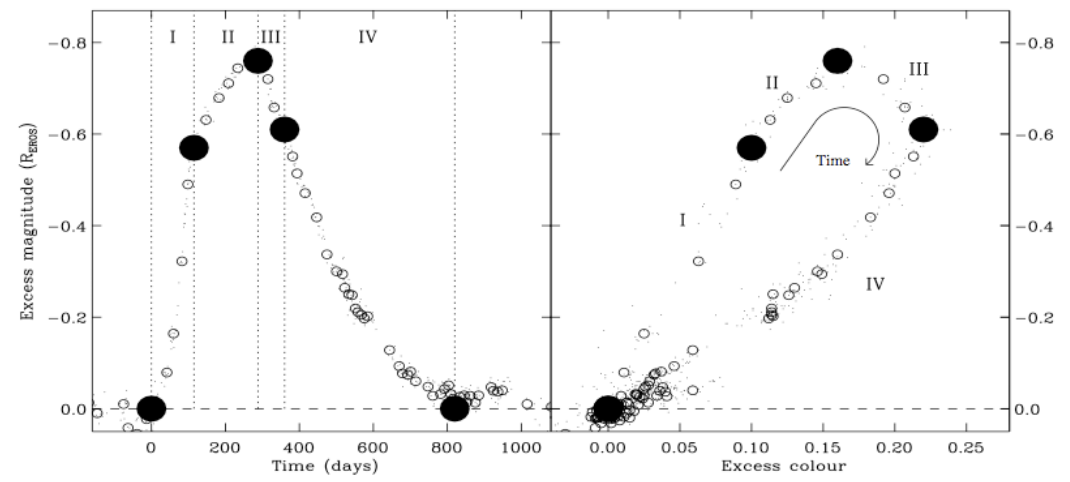

Figura 1.8: Curva de luz (painel esquerdo) e diagrama cor-magnitude (painel direito) da estrela OGLE 005209.92-731820.4 (de Wit et al., 2006).

Portanto, o diagrama da figura 1.8 mostra que, conforme a estrela aumenta seu brilho, o espectro vai se tornando cada vez mais avermelhado e, ao enfraquecer, volta a ficar azul retornando ao ponto de partida. Esta mudança na cor está associada às altas temperaturas encontradas em estrelas Be, tipicamente da ordem de $\sim 25000 \mathrm{~K}$, em contraste com a do disco $\sim 15000 \mathrm{~K}$. Com o crescimento do disco, sua cor passa a predominar o espectro global, tornando-o mais vermelho.

\subsubsection{Diagramas Cor-Polarização}

Um trabalho recente (Draper et al., 2011) estendeu a análise destes sistemas variáveis utilizando de monitoramento espectropolarimétrico. Para isto, partiram da análise da polarização intrínseca durante a transição entre as fases com e sem disco (pontos vermelhos da Fig. 1.9, painel esquerdo), tendo encontrado uma relação importante entre a variação da polarização através do valor do salto de Balmer tomado antes, $B J-$, e depois do salto, $B J+$, versus a polarização na banda $V$. Estas duas quantidades, quando tomadas uma em relação a outra, fazem surgir uma estrutura em loop com o passar do tempo (Fig. 1.9, painel direito). Este aspecto de loop, de forma similar aos diagramas cor-magnitude, surge da variação de tamanho apresentada pelo disco em suas diferentes fases, nas quais ele parte de uma situação em que possui um disco pequeno (baixa polarização) para uma com um disco maior (polarização elevada).

Por se tratar de um diagrama que representa tanto o processo de crescimento quanto o de dissipação do disco, entender os elementos que regem suas características se torna um objetivo importante. Daqui em diante, nos referiremos a ele como diagrama cor- 

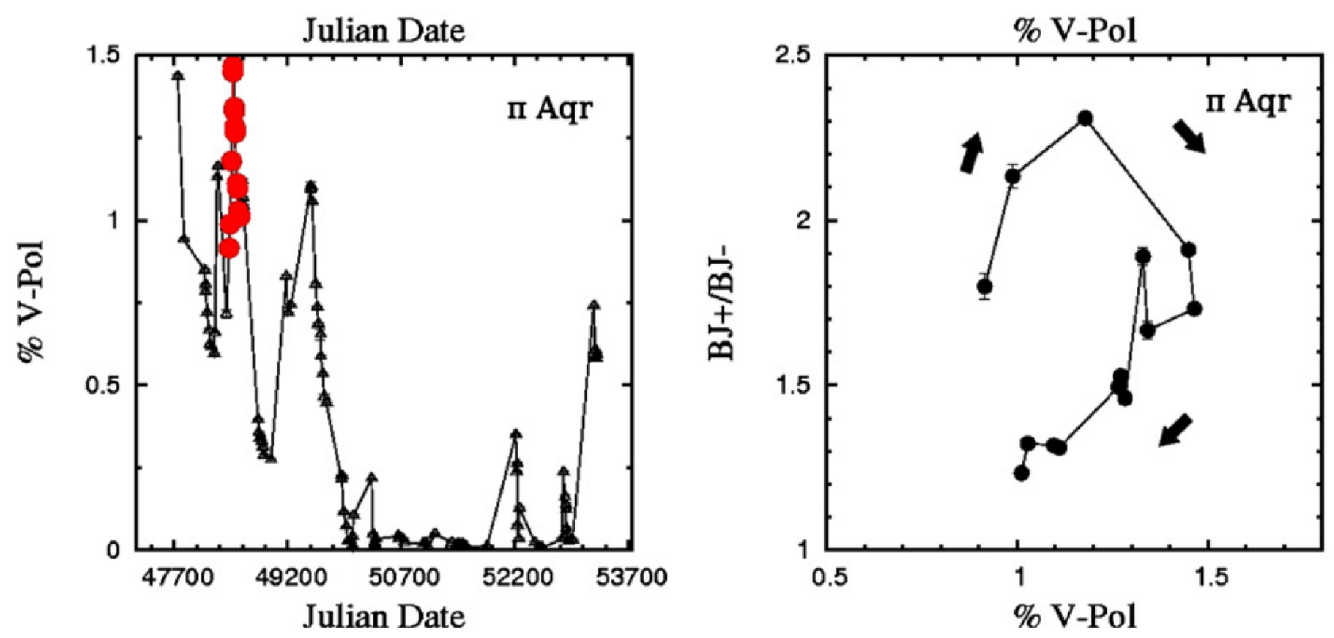

Figura 1.9: Evolução temporal da polarização intrínseca (painel esquerdo) e o diagrama cor-polarização observado (painel direito) obtido para uma período (pontos vermelhos) (Draper et al., 2011).

polarização. Essa dissertação vai tratar predominantemente da origem e características deste diagrama, visando entender porque ele é capaz de representar o ciclo de vida de um disco de Be.

\subsection{Organização Geral do Trabalho}

A dissertação foca no estudo dinâmico de estrelas Be que apresentam variabilidades marcadas pela transição entre fases com e sem disco. Para este estudo apresentamos uma análise detalhada sobre o diagrama cor-polarização inserido no contexto hidrodinâmico do disco de decréscimo viscoso. Para tal, discutimos no capítulo 2 o ferramental básico para o entendimento dos processos dinâmicos, radiativos e do código HDUST utilizado nas simulações. Seguimos apresentando nos capítulos 3 e 4 a evolução temporal da polarização para sistemas estacionários e dinâmicos por meio de modelos simplificados, explorando parâmetros de interesse, tais como a densidade de base do disco e os diferentes subtipos espectrais. Prosseguimos estudando no capítulo 5 modelos mais realistas, onde utilizamos de um código hidrodinâmico para o cálculo do perfil de densidade no disco em função do tempo, o SingleBe. Finalizamos o trabalho com o capítulo 6, onde apresentamos as conclusões. 
Capítulo 2

\section{Modelos e Ferramentas Teóricas}

\subsection{Resumo do Capítulo}

No capítulo anterior, fizemos um apanhado geral dos conceitos envolvidos em nosso estudo. Neste capítulo, aprofundamos os conceitos mais pertinentes ao nosso trabalho, estudando a astrofísica básica associada aos discos circunstelares de estrelas Be. Para isto, discutimos os processos radiativos presentes nestes discos na seção 2.2 e os conceitos de polarização da luz na seção 2.3. O modelo computacional utilizado, o código HDUst, é discutido na seção 2.4, em que apresentamos uma análise estatística do comportamento dos erros da simulação na estimativa da polarização.

\subsection{Processos Radiativos Presentes nos Discos de Be}

Iniciamos este capítulo analisando os processos pertinentes para a nossa dissertação, como os processos de espalhamento, a absorção pelo hidrogênio e os processos de emissão presentes nos discos de Be. Entender estes processos é fundamental para a compreensão dos mecanismos que controlam as propriedades do espectro polarizado das estrelas Be, tema central desta dissertação.

Para começar, imaginemos um Gedankenexperiment ${ }^{1}$, no qual uma âmpola com gás hidrogênio está à temperatura ambiente. Neste regime, a maior parte do gás estará no estado neutro, uma vez que a luz visível não é capaz de excitá-lo. Nesta configuração, veríamos a âmpola transparente quando posta contra a luz visível, ou seja, a opacidade seria baixa. Para tornar este mesmo gás opaco no visível bastaria esquentá-lo, pois, com

\footnotetext{
${ }^{1}$ Do alemão: Gedanke + Experiment, experimento mental.
} 
isso, os níveis ${ }^{2}$ superiores $(n>1)$ do hidrogênio seriam populados. A partir deste ponto, o gás passa a apresentar uma população excitada capaz de absorver a luz visível, a qual é reprocessada para comprimentos de onda diferentes. De forma análoga, podemos idealizar um sistema com gás hidrogênio recebendo radiação energética de uma fonte, por exemplo, no ultravioleta; isto aumentaria o grau de excitação, tornando o gás opaco, ou o de ionização, tornando o gás transparente. Este exercício nos fornece uma ideia dos processos que regem o comportamento radiativo de um disco de Be. Nas seções seguintes, veremos as características principais destes processos.

\subsubsection{Equilíbrio Radiativo}

De forma simplificada, um disco de Be é constituído por uma mistura de hidrogênio neutro, H I, além de prótons e elétrons. Nesta situação, podemos nos perguntar qual é a configuração de equilíbrio deste sistema e quais processos participam desta configuração. Do ponto de vista termodinâmico, queremos saber qual é a temperatura eletrônica e quais são as populações relativas dos átomos de hidrogênio ${ }^{3}$ em seus diferentes níveis e dos prótons. Para entender melhor o problema do equilíbrio radiativo, devemos, antes, distinguir entre dois conceitos: LTE e NLTE ${ }^{4}$.

\section{Equilíbrio Termodinâmico Local}

O conceito de LTE foi introduzido por Schwarzschild em 1906 em seu estudo sobre atmosferas estelares (e.g. Lopez-Puertas e Taylor, 2001). Ele notou que nenhuma quantidade de gás está isolado nestas atmosferas, desta forma, em princípio, um estado de equilíbrio não pode ser definido. Na prática, o conceito de equilíbrio é tão valioso que pode ser assumido como aproximação para alguns casos.

Para elucidar este conceito, imaginemos um pequeno volume de atmosfera composto por átomos e moléculas com níveis de energia eletrônica, vibracional e rotacional. Para um sistema isolado, o equilíbrio termodinâmico é simplesmente definido por uma temperatura cinética local constante para todo o volume. Nesta situação, o estado do gás é dito LTE se a distribuição de átomos ou moléculas no estado excitado for regida pela lei de Boltzman

\footnotetext{
${ }^{2}$ Adotamos a terminologia nível para expressar o número quântico principal do átomo de hidrogênio.

${ }^{3}$ Nos restringimos apenas ao hidrogênio, pois este é o único elemento presente no HDUST no momento.

${ }^{4}$ Do inglês: Local Thermodynamic Equilibrium e Non-Local Thermodynamic Equilibrium.
} 
com a temperatura cinética local. Neste regime, as propriedades radiativas da matéria para a transição entre estados podem ser descritas apenas em termos da temperatura; em particular, a função fonte da transição torna-se igual a função de Planck naquela temperatura. Desta forma, a emissividade $j_{\nu}$ terá um valor fornecido pela lei de Kirchhoff (e.g. Shu, 1991).

\section{Equilíbrio Termodinâmico Não-Local}

Quando consideramos a presença de uma ou mais fontes de radiação, a distribuição de população dos níveis será dominada por processos radiativos de absorção e emissão de fótons, sendo, em geral, regida por uma distribuição e espectro que não apresentam relação alguma com a função de Planck. Isto constitui o caso NLTE, uma vez que o campo de radiação é não-local. Em discos de estrela Be, a temperatura eletrônica e as populações do hidrogênio são regidas pelo caso NLTE. Este problema complexo é resolvido pelo código HDUST, discutido na seção 2.4 .

\subsubsection{Processos de Emissão e Absorção}

Vejamos, agora, os processos de emissão: recombinação, bremsstrahlung e desexcitação; e seus processos de absorção correspondentes: fotoionização, bremsstrahlung inverso e excitação; presentes nesses sistemas. O processo de recombinação e bremsstrahlung, tal como seus inversos, são responsáveis pela emissão no contínuo, enquanto que os de desexcitação e excitação são responsáveis pelo surgimento de linhas espectrais.

\section{Recombinação e Fotoionização}

Um dos processos responsáveis pela emissão num disco de Be é a recombinação ou emissão livre-ligado, processo pelo qual íons do plasma capturam elétrons livres para formar átomos neutros. Seu inverso é o processo de fotoionização ou absorção ligado-livre, o qual representa a liberação de elétrons por seus átomos. Tal processo pode ser verificado pela presença de descontinuidades nos espectros estelares, semelhantes aos vistos nos espectros polarizados apresentados na seção 1.4.1. Verificamos o aumento da opacidade do material em comprimentos de onda mais curtos que os comprimentos críticos das descontinuidades. Detalhes deste comportamento serão vistos no capítulo 3. 


\section{Bremsstrahlung e Bremsstrahlung Inverso}

A emissão bremsstrahlung ou emissão livre-livre é um processo de interação de elétrons livres com os campos gerados por prótons ou íons. Para que este processo seja relevante é requerida uma quantidade grande de elétrons livres sendo, por isto, mais significativo à altas temperaturas. Seu processo inverso é conhecido como bremsstrahlung inverso ou absorção livre-livre, caracterizado pela captura de um fóton por dois elétrons. Tal processo apresenta uma profundidade óptica proporcional ao quadrado do comprimento de onda, sendo importante para comprimentos de onda mais longos.

\section{Desexcitação e Excitação}

Enfim, temos a emissão devida ao hidrogênio neutro, a emissão ligado-ligado, manifestada pela emissão de um fóton por um átomo apenas em certos comprimentos de onda. Seu processo inverso é a excitação radiativa ou absorção ligado-ligado, na qual a absorção dos fotons devida à transição dos elétrons para níveis eletrônicos superiores é a responsável pela formação de linhas de absorção, gerando absorção em comprimentos de onda bem estabelecidos pela equação de Rydberg (e.g. Eisberg e Resnick, 1985).

\section{Transições Colisionais}

Também é possível para um elétron em um átomo fazer uma transição de um nível $i$ para $j$ durante uma colisão de um átomo com uma partícula alvo. A taxa de excitação $C_{\mathrm{ij}}$ (e desexcitação $C_{\mathrm{ji}}$ ) são definidas em termos da uma seção de choque colisional dependente da velocidade $\sigma_{\mathrm{ij}}(v)$. Para maiores detalhes recomendamos a leitura de Padmanabhan (2000).

Portanto, num sistema como o disco de uma estrela Be, a energia cinética do gás do disco aumenta devido à radiação estelar, gerando um sistema onde o processo de ionização por colisão ou da interação dos elétrons com quantidades discretas de energia (transições ligadolivre e transições ligado-ligado) influenciam a transferência radiativa. Estes processos agem gerando elétrons livres ou transições eletrônicas que vão aumentando em número conforme a temperatura cresce ou, de forma análoga, quando vamos de subtipos espectrais mais tardios para os mais recentes. 


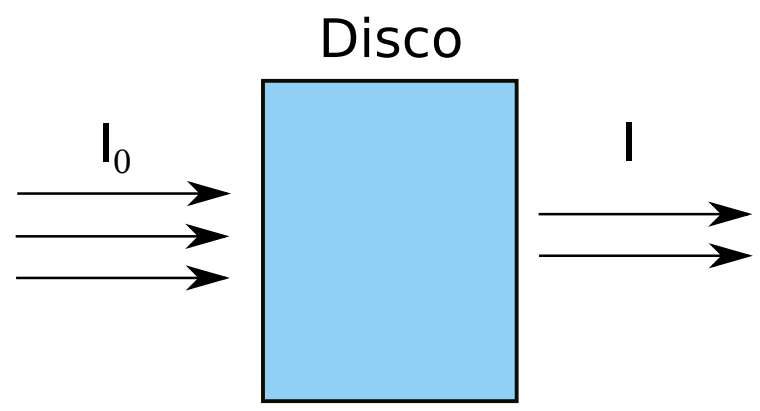

Figura 2.1: Esquema representativo do problema de transporte radiativo.

\subsubsection{Opacidade}

Conhecidos os processos principais de absorção, pode-se calcular a opacidade total em certa parte do disco somando-se a contribuição de cada uma das transições discutidas somada aos processos de espalhamento (visto na seção 2.3). Portanto, poderíamos representar a opacidade total, $\kappa_{\nu}$, como,

$$
\kappa_{\nu}=\kappa_{\nu}^{\mathrm{ff}}+\kappa_{\nu}^{\mathrm{bf}}+\kappa_{\nu}^{\mathrm{bb}}+\kappa_{\mathrm{el}}
$$

onde os prefixos ff, bf, bb e el, representam os processos livre-livre, ligado-livre, ligadoligado e por espalhamento eletrônico. Foi mantida a notação em inglês para evitar ambiguidade.

No capítulo 3, retornaremos o estudo das opacidades, porém focando nas opacidades específicas nos comprimentos de onda dos saltos de Balmer e Paschen.

\subsubsection{Equação de Transferência Radiativa}

Quando um feixe de luz de frequência $\nu$ incide sobre um volume preenchido com uma certa quantidade de matéria, a intensidade específica emergente, $I_{\nu}$, pode ser maior ou menor que a incidente, $I_{\nu}(0)$, dependendo dos processos de espalhamento, emissão e absorção radiativos que ocorrerão (Fig. 2.1). Por esta razão, definir uma equação que englobe os efeitos dos processos radiativos se torna fundamental. Para isto, devemos incluir na solução os três efeitos responsáveis por mudar a intensidade: emissão, absorção e espalhamento. 
Neste problema, a emissão é representada pela função fonte (Rybicki e Lightman, 1979),

$$
S_{\nu} \equiv \frac{j_{\nu}}{\alpha_{\nu}}
$$

onde $j_{\nu}$ é a emissividade específica.

A absorção e o espalhamento são representados pela absortividade ${ }^{5}, \alpha_{\nu}$, ou pela opacidade, $\kappa_{\nu}$. As dependências destas grandezas é apresentada no apêndice C. Estas grandezas estão relacionadas ao conceito de profundidade óptica, uma grandeza que mede a probabilidade de absorção de um feixe de luz. Matematicamente, a definimos (Rybicki e Lightman, 1979) como,

$$
\tau_{\nu}(s)=\int_{0}^{s} \rho \kappa_{\nu}\left(s^{\prime}\right) d s^{\prime}=\int_{0}^{s} \alpha_{\nu}\left(s^{\prime}\right) d s^{\prime}
$$

onde $d s$ é a espessura do meio e $\rho$ é a densidade volumétrica ${ }^{6}$ de partículas. Portanto, um meio totalmente transparente possui uma probabilidade nula de absorção.

Desta forma, podemos representar a equação de transferência radiativa como,

$$
\frac{d I_{\nu}}{d \tau_{\nu}}=S_{\nu}-I_{\nu}
$$

cuja solução é,

$$
I_{\nu}\left(\tau_{\nu}\right)=I_{\nu}(0) e^{-\tau_{\nu}}+S_{\nu}\left(1-e^{-\tau_{\nu}}\right) .
$$

Esta equação, válida em meios homogêneos, engloba tanto a extinção, $I_{\nu}(0) e^{-\tau_{\nu}}$, quanto a emissão e a auto-absorção, $S_{\nu}\left(1-e^{-\tau_{\nu}}\right)$. As diferentes opacidades (ligado-livre, livre-livre e eletrônica) que contribuem para o termo $\tau_{\nu}$ são apresentadas no apêndice C. No próximo capítulo, apresentamos uma análise detalhada de suas dependências.

\subsection{Conceitos Básicos de Polarização}

Geralmente, estrelas emitem radiação não polarizada. Entretanto, há casos especiais em que a radiação pode tornar-se parcialmente polarizada. Um exemplo, é a polarização

\footnotetext{
${ }^{5} \mathrm{~A}$ absortividade é fornecida por $\alpha_{\nu}=n \sigma_{\nu}=\rho \kappa_{\nu}$, onde $n$ é a densidade numérica de partículas, $\sigma_{\nu}$ é a seção de choque específica, $\rho$ é a densidade de massa e $\kappa$ a opacidade.

${ }^{6} \mathrm{~A}$ densidade volumétrica pode ser relacionada com a densidade numérica de partículas da seguinte maneira, $\rho=n \cdot m_{\mathrm{H}} / \mu$, onde $\mu$ é a massa molecular relativa (e.g. Isaacs et al., 2003).
} 
por espalhamento Thomson nos discos de estrelas Be. A seguir, apresentamos os casos especiais da polarização elíptica (a circular e a linear) e os processos de polarização da luz, com o propósito de entender como a polarização surge em discos de Be e por que ela é perpendicular ao plano equatorial destes discos.

\subsubsection{Polarização}

Ondas eletromagnéticas monocromáticas ${ }^{7}$ podem ser polarizadas, isto é, seus vetores campo elétrico e magnético, $\vec{E}$ e $\vec{B}$, podem apresentar um padrão coerente $^{8}$ em relação à direção de propagação. Podemos caracterizar estas ondas pelo ângulo $\chi$ que seu vetor campo elétrico faz com um eixo $x$ arbitrário, por sua amplitude $E_{0}$ e pelo ângulo $\beta$, relacionado com a excentricidade (Fig. 2.2). Podemos obter as componentes do vetor campo elétrico de uma onda elipticamente polarizada com o auxílio da figura 2.2. Para isto, transformamos as componentes, $E_{0} \cos (\beta, t)$ e $E_{0} \sin (\beta, t)$, para o sistema $x-y$, encontrando a equação horária de uma onda elipticamente polarizada sobre um ponto fixo do eixo de propagação, $z$,

$$
\vec{E}=\left\{\begin{array}{l}
\vec{E}_{x}(t)=\hat{i} E_{0}(\cos \beta \cos \chi \cos \omega t+\sin \beta \sin \chi \sin \omega t)=E_{0_{x}} \cos \left(\omega t-\phi_{x}\right), \\
\vec{E}_{y}(t)=\hat{j} E_{0}(\cos \beta \sin \chi \cos \omega t-\sin \beta \cos \chi \sin \omega t)=E_{0_{y}} \cos \left(\omega t-\phi_{y}\right)
\end{array}\right.
$$

onde $t$ é o instante de tempo, $E_{0 x}$ e $E_{0 y}$ são as amplitudes e, $\phi_{x}$ e $\phi_{y}$, as fases das componentes.

As polarizações linear e circular são casos especiais da elíptica. Das equações anteriores, vemos que,

$$
-\frac{\pi}{2} \leq \beta \leq \frac{\pi}{2}=\left\{\begin{array}{l}
\beta=0 \text { ou } \pi / 2, \text { polarização linear; } \\
0<\beta<\pi / 2, \text { polarização esquerda; } \\
-\pi / 2<\beta<0, \text { polarização direita. }
\end{array}\right.
$$

Portanto, a polarização linear ou plano-polarizada constitui o caso em que a amplitude do campo elétrico é constante, ou seja, suas componentes estão em fase apresentando

\footnotetext{
${ }^{7}$ Frequência angular fixa, $\omega=2 \pi \nu=$ const.

${ }^{8}$ Correlação entre as fases das componentes do campo elétrico.
} 


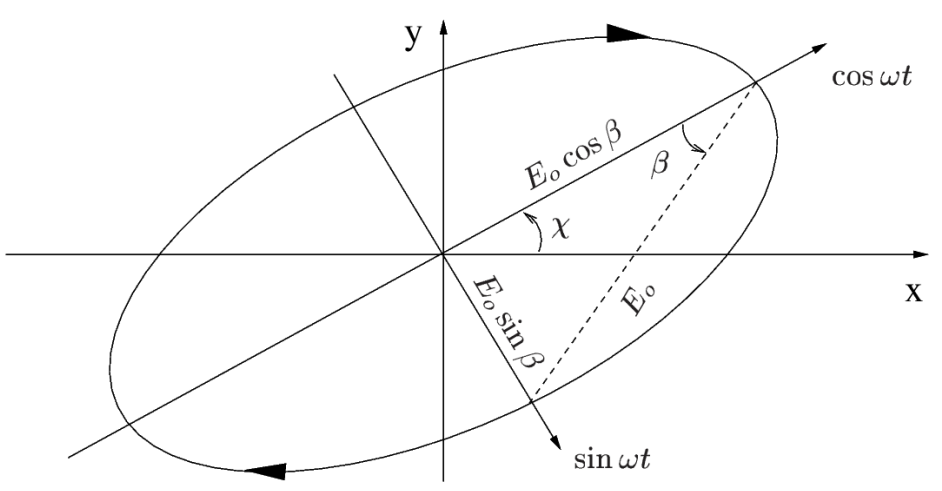

Figura 2.2: Representação de uma onda elipticamente polarizada vista de frente Ernie Seaquist (2003), Curso de Processos Radiativos.

seus máximos e mínimos ao mesmo tempo. No caso da polarização circular, as componentes possuem magnitudes iguais e estão defasadas em $90^{\circ}$. Podemos, ainda, classificar a polarização circular em dois tipos, a partir do sentido em que o plano de vibração está rotacionando durante um período, assim diz-se que a onda está polarizada à direita ou à esquerda.

\section{Os Parâmetros de Stokes}

Os parâmetros de Stokes são somas e diferenças da intensidade do campo de radiação medido ao longo de diferentes eixos (Stokes, 1852). Por definição, os parâmetros de Stokes são:

$$
\begin{array}{rlrl}
I & \equiv E_{0_{x}}^{2}+E_{0_{y}}^{2}=E_{0}^{2} & & \text {, intensidade total } \\
Q & \equiv E_{0_{x}}^{2}-E_{0_{y}}^{2}=E_{0}^{2} \cos 2 \beta \cos 2 \chi & & \text {, polarização linear } \\
U & \equiv 2 E_{0_{x}} E_{0_{y}} \cos \left(\phi_{x}-\phi_{y}\right)=E_{0}^{2} \cos 2 \beta \sin 2 \chi & & \text {, polarização linear } \\
V & \equiv 2 E_{0_{x}} E_{0_{y}} \sin \left(\phi_{x}-\phi_{y}\right)=E_{0}^{2} \sin 2 \chi & , \text { polarização circular }
\end{array}
$$

É fácil ver que, 


$$
\begin{aligned}
\tan 2 \chi & =U / Q ; \\
\sin 2 \beta & =V / I ; \\
I^{2} & \equiv Q^{2}+U^{2}+V^{2} .
\end{aligned}
$$

Pictoricamente, podemos representar os parâmetros de Stokes como somas e diferenças da componente polarizada em diferentes direções:

$$
\begin{gathered}
I=\uparrow+\leftrightarrow ; \\
Q=\uparrow-\leftrightarrow ; \\
U=\swarrow-\nwarrow ; \\
V=\circlearrowleft-\circlearrowright .
\end{gathered}
$$

Fisicamente, I representa a intensidade total do campo de radiação, $Q$ a diferença entre as intensidades ao longo de dois eixos perpendiculares, $U$ a diferença de intensidade ao longo de dois eixos rotacionados de $45^{\circ}$ em relação à direção $Q$ e $V$ a diferença na intensidade entre as componentes circularmente polarizadas.

\subsubsection{Processos de Polarização da Luz}

Um feixe de luz nã0-polarizado pode tornar-se polarizado por três processos: reflexão, absorção e espalhamento. A polarização por reflexão ocorre quando uma superfície reflete um feixe de luz não-polarizada gerando um feixe refletido polarizado. Este tipo de polarização pode ocorrer em superfícies sólidas de planetas e em corpos menores do Sistema Solar. No caso da polarização por absorção, a polarização surge da absorção direcional de certas componentes de um feixe não polarizado ao atravessar um meio material. Por sua vez, a polarização por espalhamento no óptico pode ocorrer por três processos:

1. Espalhamento Rayleigh ocorre quando a radiação incidente interage com partículas carregadas ou moléculas cujo tamanho sejam muito menores do que o comprimento de onda da radiação incidente (ex. moléculas em atmosferas de estrelas frias e planetas);

2. Espalhamento Mie se deve à interação da radiação com grãos com tamanhos físicos da ordem do comprimento de onda (ex. poeira interestelar); 
3. Espalhamento Thomson ou eletrônico ocorre quando uma carga livre irradia em resposta a uma onda eletromagnética incidente. Se a carga oscilar em regimes não relativísticos, $h \nu /\left(m c^{2}\right) \ll 1$, pode-se demonstrar que não ocorre mundança no comprimento de onda dos fótons espalhados (ex. elétrons livres em envelopes de estrelas).

\section{O Espalhamento Thomson ou Eletrônico}

Elétrons isolados não absorvem energia, mas podem espalhar a radiação. Quando radiação atinge elétrons livres, estes redistribuem (espalham) a luz de acordo com (Rybicki e Lightman, 1979),

$$
\left(\frac{d \sigma}{d \Omega}\right)=r_{0}^{2} \sin ^{2} \chi=\left(\frac{3}{8 \pi} \sigma_{\mathrm{T}}\right)^{1 / 2} \sin ^{2} \chi
$$

onde,

$$
r_{0} \equiv \frac{e^{2}}{m_{\mathrm{e}} c^{2}}
$$

é o raio do elétron, $\sigma_{\mathrm{T}}$ é a seção de choque de Thomson e $\chi$ é o ângulo de espalhamento (Fig. 2.3). As relações acima apresentam uma característica importante deste processo, sua independência com o comprimento de onda. Além disso, assim como no caso das transições livre-livre, o processo de espalhamento Thomson domina apenas a altas temperaturas, regime dominado por mais elétrons livres.

\section{Polarização por Espalhamento Thomson em Envelopes Circunstelares}

Descrever o espalhamento da luz por um elétron é relativamente simples, porém para modelar um disco de Be devemos levar em conta o espalhamento por elétrons distribuídos em um dado volume. Muitos trabalhos foram conduzidos até chegarmos numa teoria de espalhamento em discos de Be que fosse consistente com as observações. A seguir, discutimos brevemente os principais trabalhos responsáveis pelo panorama atual.

O primeiro estudo relevante do problema foi apresentado por Brown e McLean (1977). Estes autores expressaram analiticamente a polarização no caso de um envelope com simetria axial não-esférico e opticamente fino; com isso, verificaram que a polarização líquida depende de três parâmetros do envelope: uma média da profundidade óptica eletrônica, $\bar{\tau}$, 


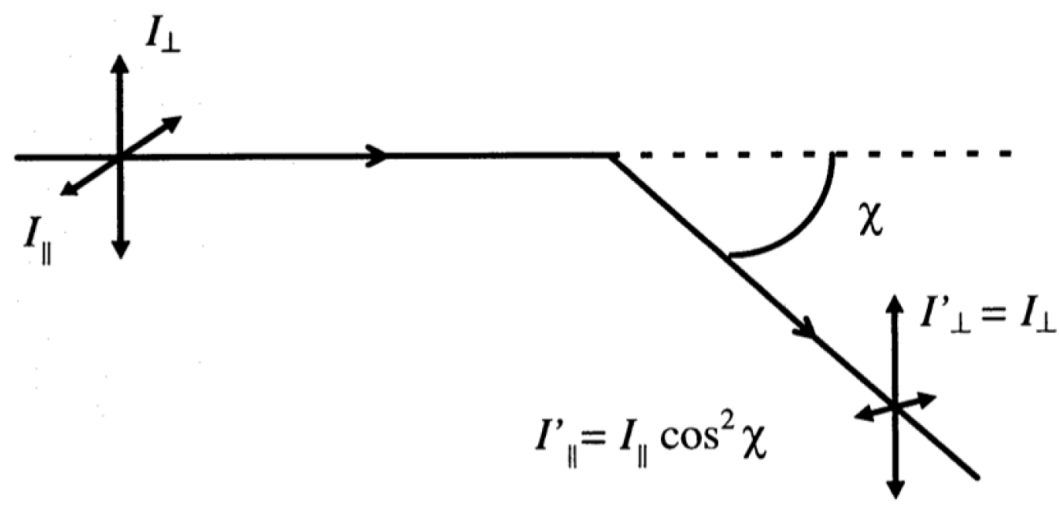

Figura 2.3: Representação do processo de espalhamento Thomson (Wood et al., 1996b).

um fator de forma, $\gamma$, e a inclinação entre o eixo de simetria e a linha de visada, $i$, obtendo assim,

$$
P=\bar{\tau}(1-3 \gamma) \sin ^{2} i
$$

Dessa forma, a polarização seria máxima para $i=90^{\circ}$. Posteriormente, Fox (1991) verificou uma redução significativa no nível de polarização após incluir processos de absorção na análise. Em análise posterior, que incorporou efeitos importantes como o espalhamento múltiplo (apêndice E), Wood et al. (1996a) mostraram que a dependência de $P$ com $i$ é bem mais complexa, estando relacionada com o fenômeno de auto-absorção, em que parte do fluxo espalhado na parte interna do disco e espalhado em direções perpendiculares ao equador é absorvida pelo disco, o que diminui o fluxo polarizado e, portanto, o nível de polarização.

Já sabemos o que controla o nível de polarização nos discos de Be; falta explicar a orientação do vetor de polarização nestes discos. No caso da polarização por espalhamento Thomson, inicialmente podemos pensar na radiação composta por duas componentes, $I_{\|}$ e $I_{\perp}$ (Fig. 2.3). Ao encontrar um elétron, este espalhará, para um ângulo $\chi$, uma intensidade $I_{\|}^{\prime}=I_{\|} \cos ^{2}(\chi)$ e $I_{\perp}^{\prime}=I_{\perp}$. Em outras palavras, quando a luz não polarizada sofre espalhamento Thomson, ela torna-se polarizada perpendicular ao plano de espalhamento ${ }^{9}$ (Wood et al., 1996b). Após o espalhamento, a amplitude do campo elétrico é reduzida por $\cos ^{2} \chi$. Enquanto isso, a componente perpendicular ao plano permanece inalterada.

\footnotetext{
${ }^{9}$ Plano que contém a radiação incidente e a espalhada.
} 


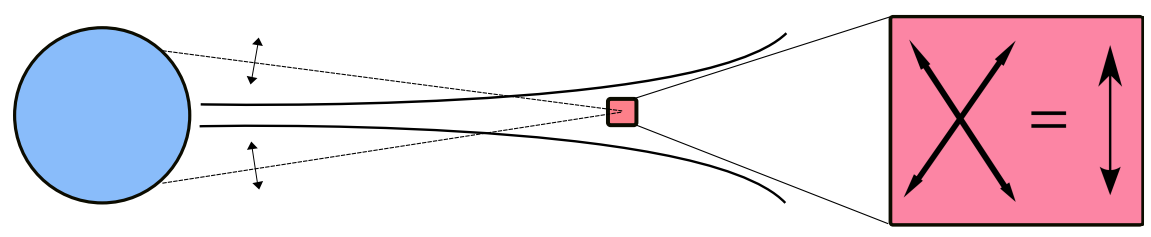

Figura 2.4: Esquema representativo de como ocorre a orientação da polarização num disco de Be, mostrase o espalhamento a $90^{\circ}$ da luz proveniente de diferentes hemisférios. As componentes paralelas, $I_{\|}$, dos feixes espalhados são, por definição, nulos. O quadro maior em vermelho representa uma ampliação de uma região pequena do disco, junto com os vetores de polarização dos dois feixes considerados.

Utilizando esta notação, o grau de polarização será ${ }^{10}$,

$$
p=\frac{I_{\perp}-I_{\|}}{I_{\perp}+I_{\|}}=\frac{1-\cos ^{2} \chi}{1+\cos ^{2} \chi} .
$$

Agora, podemos analisar a radiação proveniente de um pequeno volume de um disco de Be. Para isto, representamos na figura 2.4 dois feixes não-polarizados (linhas tracejadas) atingindo a mesma região (quadrado vermelho). Pela geometria, a fração de cada feixe espalhada em nossa direção terá uma componente $Q$ e uma componente $U$. As componentes $U$ se cancelam, restando a componente $Q$ que é perpendicular ao plano do disco.

\subsection{HDUST}

O HDust utiliza o método de Monte Carlo para fornecer uma solução formal, dentro de um erro estipulado, para a equação de transporte radiativo, incluindo a polarização, efeitos de espalhamento múltiplo, condições de equilíbrio radiativo e estatístico em regime NLTE para os níveis de população do hidrogênio em meios com geometria tridimensional e cinemática arbitrária. As fontes de opacidade atualmente implementadas são:

1) hidrogênio com números de ocupação NLTE;

2) espalhamento Thomson;

3) brehmsstrahlung;

4) grãos de poeira, se presentes, usando a teoria de Mie.

\footnotetext{
${ }^{10} \mathrm{~A}$ dependência angular da polarização é obtida lembrando-se que $I_{\perp}=\left(E_{0} / 2\right)^{2}$ e $I_{\|}=\left(E_{0} / 2\right)^{2} \cos ^{2} \chi$.
} 
Uma característica importante do código é sua capacidade de resolver simultaneamente a condição de equilíbrio radiativo e as equações de fluido do disco, tal que a estrutura geométrica deste pode ser obtida de forma auto-consistente com o transporte da radiação. O código também inclui uma descrição muito realista da estrela, levando em conta efeitos como o escurecimento gravitacional e a deformação rotacional, importantes para estrelas com alta taxa de rotação. A saída do código consiste em imagens, fluxos e polarização para qualquer intervalo espectral desejado.

\subsubsection{Determinação dos Erros}

O método de Monte Carlo utiliza técnicas de amostragem desenvolvidas pelo orientador, cujo comportamento estatístico depende dos parâmetros físicos do sistema estudado. Visando entender melhor a questão do erro nas simulações, consideramos importante iniciar nosso estudo por uma análise estatística detalhada da polarização; com este estudo, fundamentamos a questão do erro estatístico em nossos modelos, bem como determinamos o número de fótons mínimo para se obter uma boa estatística para as simulações. 


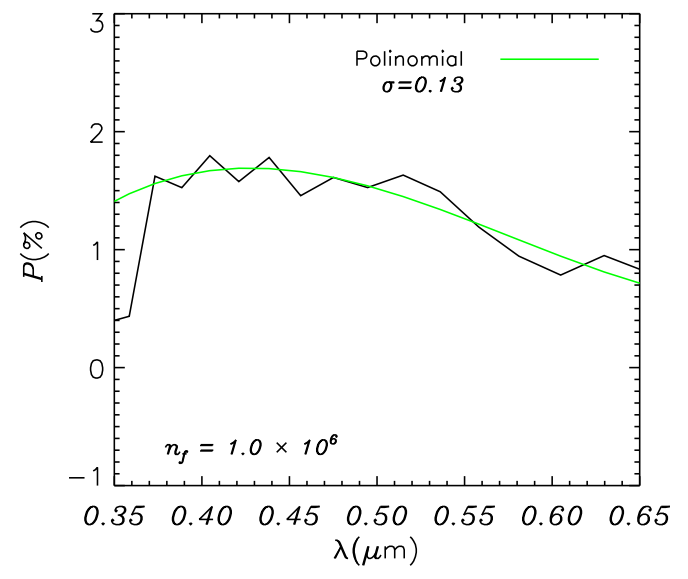

(a)

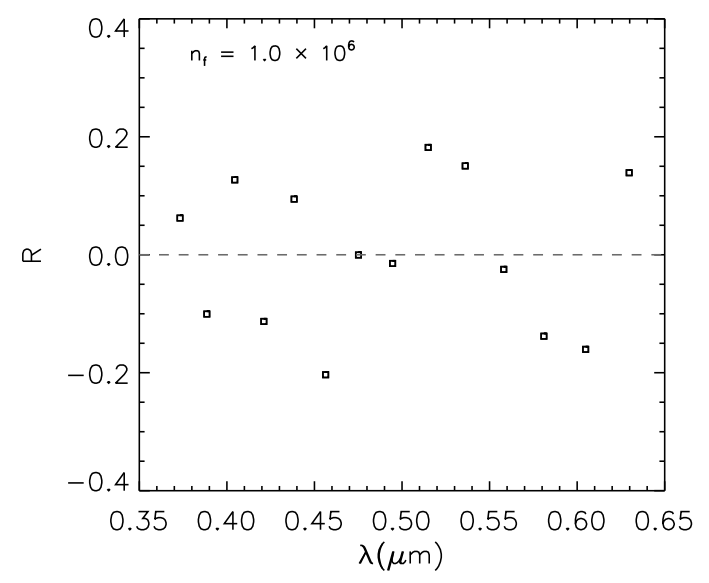

(c)

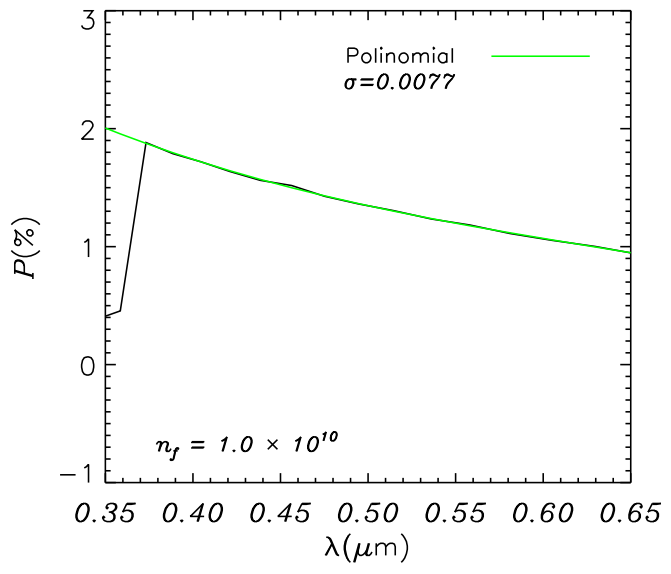

(b)

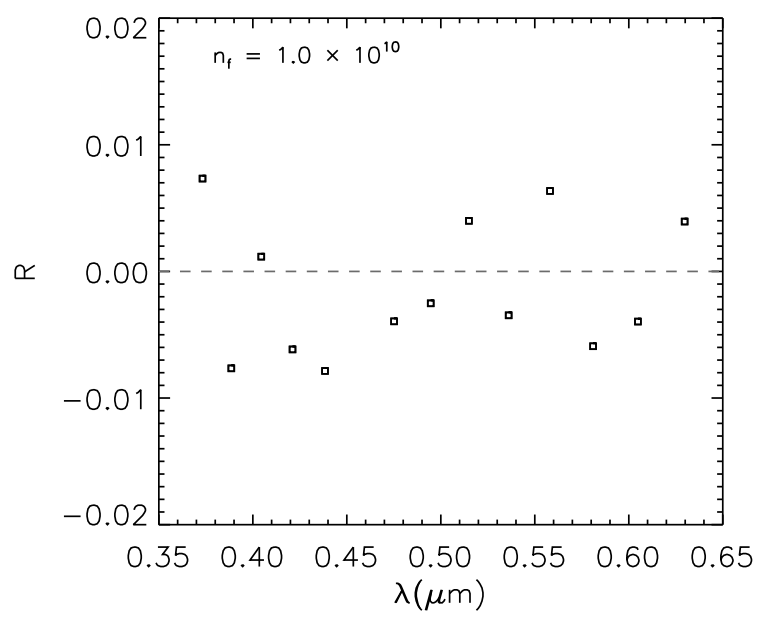

(d)

Figura 2.5: Topo: Espectros polarizados simulados com diferentes valores de fótons, $n_{\mathrm{f}}$, juntamente com os ajustes polinomias (curvas verdes) para uma estrela de subtipo espectral B2 com disco a um ângulo de inclinação de $70^{\circ}$. Abaixo: Gráficos dos resíduos, $R$, dos respectivos ajustes polinomiais. 
Em princípio, a estatística da polarização depende do número de fótons espalhados, cujo valor resulta de uma combinação complexa entre as características físicas da estrela e do disco e, portanto, não pode ser conhecido a priori. Para fundamentar a escolha de um número suficiente de fótons em nossas simulações, estudamos o espectro polarizado de um modelo típico para diferentes número de fótons. A partir destes valores, selecionamos a quantidade de fótons mínima necessária para a obtenção de uma precisão de uma certa porcentagem.

Podemos empregar dois procedimentos distintos para estimar o erro da polarização. O primeiro parte do princípio de que duas simulações com diferentes sementes do gerador de número aleatórios serão estatísticamente independentes. Dessa forma, pode-se realizar um certo número de simulações independentes e fazer um desvio padrão da média dos resultados. Uma forma mais simples, mas não tão rigorosa, emprega o resultado de apenas uma simulação, da seguinte forma: ajusta-se um polinômio a uma parte do espectro polarizado sem linhas de emissão, subtrai-se o ajuste do espectro e calcula-se o desvio padrão do resultado. Na ausência de viés importante no ajuste, este método permite obter uma estimativa rápida do erro da polarização a partir de apenas uma simulação. Este procedimento foi aplicado na região do visível entre 3700 e $6500 \AA$ e está ilustrado na figura 2.5. Para uma simulação com $10^{6}$ fótons (Fig. 2.5(a)), a dispersão é bastante alta $(\sim 0.13 \%)$, caindo para $\sim 0.0077 \%$ para $10^{10}$ fótons (Fig. 2.5(b)).

Na figura 2.6, mostramos como a dispersão da polarização varia com o número de fótons $\left(n_{\mathrm{f}}\right)$ utilizado na simulação. Espera-se que a dispersão caia com o aumento de $n_{\mathrm{f}}$ da seguinte forma: $\sigma \propto n_{\mathrm{f}}^{1 / 2}$ (estatística de Poisson). Realmente, vemos que para $n_{\mathrm{f}}>10^{8}$ a dispersão estimada pelo procedimento acima aproxima-se da dependência funcional esperada, mostrada pela linha vermelha. Estes resultados indicam que acurácias próximas a $0.01 \%$ em polarização podem ser obtidas com aproximadamente $10^{9}$ fótons. Uma análise para estrelas de diferentes subtipos espectrais mostrou que estrelas de subtipo espectral recente requerem mais fótons para uma mesma dispersão no espectro polarizado. Isto está relacionado com o fato do pico em emissão estar mais deslocado para a região ultravioleta do espectro, ou seja, o fluxo no visível se torna menor para estas estrelas.

Este estudo permitiu determinar, para os modelos simulados, que o número excelente de fótons a rodar deve satisfazer $10^{9} \leq n_{\mathrm{f}} \leq 10^{10}$. 


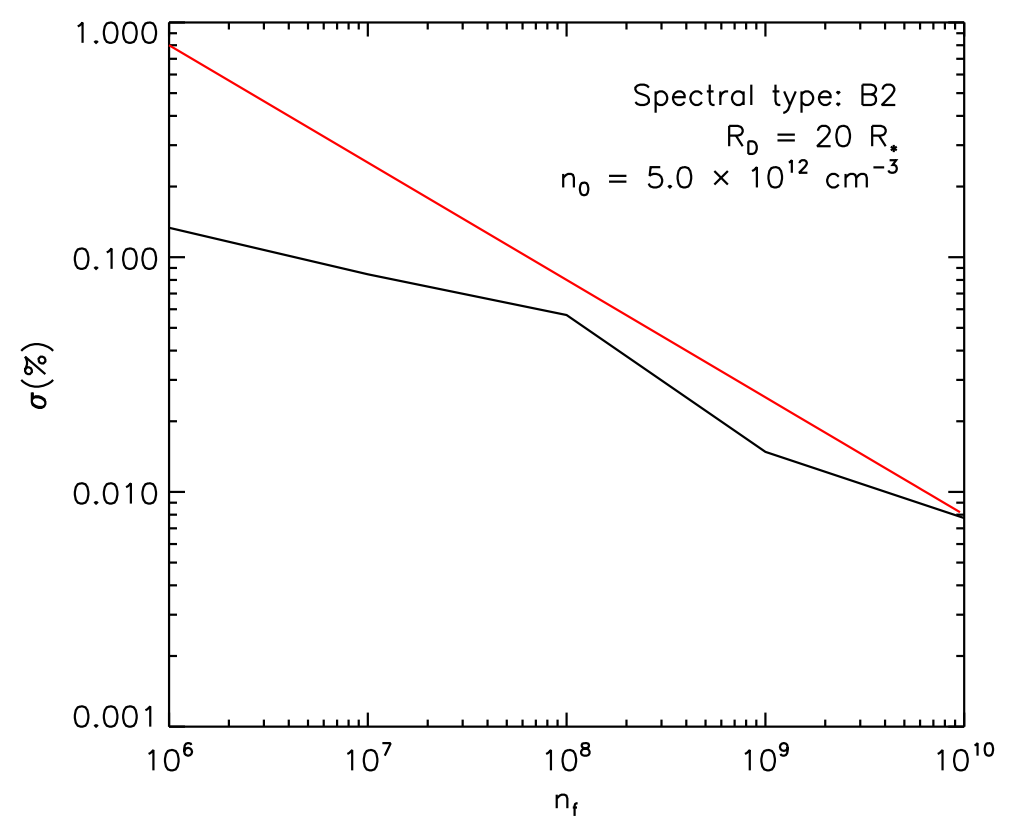

Figura 2.6: (a) Gráfico da dispersão em função da quantidade de fótons $\left(\sigma \times n_{\mathrm{f}}\right)$ para uma estrela de subtipo espectral B2 com densidade na base $5.0 \times 10^{12} \mathrm{~cm}^{-3}$ e tamanho do envelope de $20 R_{\star}$. A curva vermelha representa o ajuste $\propto \sqrt{n_{\mathrm{f}}}$. 
Capítulo 3

\section{Discos de Estrelas Be em Estado Estacionário}

\subsection{Resumo do Capítulo}

O objetivo deste capítulo é aprofundar o conhecimento sobre como as propriedades físicas dos discos de Be se relacionam com as estelares. Com isso, visamos entender como os parâmetros livres adotados influenciam os observáveis de interesse; para atingi-lo, cumprimos três passos preliminares: o primeiro foi a construção do modelo de disco estacionário (seção 3.2); em seguida, realizamos uma análise inédita das propriedades térmicas do disco para diferentes subtipos espectrais (seção 3.3). Na seção 3.4 estudamos as absotividades do disco e em seguida, na seção 3.5, apresentamos um estudo da variação das características polarimétricas em discos em estado estacionário para os diferentes parâmetros livres adotados.

\subsection{O Modelo Utilizado}

Neste capítulo, estudaremos modelos de disco de estrela Be em estado estacionário (i.e., independente do tempo). Como visto na seção 1.4.2, trata-se de um modelo que prevê uma estrutura relativamente simples para o disco, que consiste de um perfil de densidade radial dado por uma lei de potência, eq. 1.1, e um perfil gaussiano na direção vertical, com uma escala de altura fornecida pela equação 1.2.

Com este estudo inicial, buscaremos compreender as propriedades termodinâmicas do disco, bem como entender de que forma os principais parâmetros livres influenciam o espectro emergente, em particular, o espectro polarizado. 


\subsubsection{Parâmetros Adotados}

O modelo consiste de uma estrela circundada por um disco circunstelar em estado estacionário. Estrelas Be são notórias por sua rápida rotação. Para incluir este importante efeito físico nos modelos, consideramos estrelas com taxas de rotação de $80 \%$ da velocidade angular crítica (Eq. 1.3). Tal rotação corresponde a um achatamento de $R_{\text {eq }} / R_{\text {polo }}=1.14$ e uma razão entre a temperatura efetiva do polo e do equador calculada a partir do teorema de von Zeipel que especifica que o fluxo dirigido de um envelope radiativo é proporcional à gravidade superficial local, $T_{\mathrm{ef}} \propto g_{\mathrm{ef}}^{0.25}$ (Collins, 1963).

Para nosso estudo, consideramos valores de temperatura efetiva, massa e luminosidade, característicos de cada subtipo espectral. Adotamos estrelas de subtipos espectrais de B0 a B5, mantendo as propriedades geométricas, $R_{\star}$ basicamente, fixadas nas características de uma estrela de subtipo espectral B2 ${ }^{1}$. Com isto, produzimos sistemas físicos de mesmo tamanho para todos os subtipos espectrais estudados, tornando a comparação entre os resultados mais significativa. Consideramos discos de hidrogênio puro e avaliamos diferentes escalas de densidade e ângulos de inclinação. Em síntese, avaliamos os seguintes parâmetros livres:

i) Densidade na base do disco, $n_{0}$;

ii) Raio do disco, $r_{\mathrm{d}}$;

iii) Subtipo espectral;

iv) Ângulo de inclinação do disco, $i$;

v) Escala de altura do disco, $H$.

Como vimos no capítulo 1 , os discos são sustentados na direção vertical pela pressão do gás, ou seja, são controlados pela temperatura; por outro lado, como veremos na seção 3.3, os discos apresentam uma distribuição radial não isotérmica. Neste estudo inicial, simplificamos a análise considerando uma estrutura vertical isotérmica, ou seja, adotamos uma temperatura média do gás que regerá a escala de altura. Carciofi e Bjorkman (2006)

\footnotetext{
${ }^{1}$ Por ser o subtipo espectral mais comum na amostra de estrelas Be conhecidas (Porter e Rivinius, 2003).
} 


\begin{tabular}{|c|c|c|c|c|c|c|}
\hline Subtipo Espectral & B0 & B1 & B2 & B3 & B4 & B5 \\
\hline$T_{\mathrm{ef}}^{\text {polo }}(\mathrm{K})$ & 29900 & 26200 & 23100 & 19100 & 17200 & 15500 \\
\hline$v_{\text {crit }}(\mathrm{km} / \mathrm{s})$ & 660 & 530 & 585 & 466 & 447 & 429 \\
\hline$L\left(\mathrm{~L}_{\odot}\right)$ & 24200 & 10200 & 4400 & 1500 & 830 & 467 \\
\hline$M\left(\mathrm{M}_{\odot}\right)$ & 14.6 & 11.0 & 8.6 & 6.1 & 5.1 & 4.4 \\
\hline$R_{\mathrm{eq}}\left(\mathrm{R}_{\odot}\right)$ & 5.8 & 4.9 & 4.3 & 3.6 & 3.3 & 3.0 \\
\hline$\lambda_{\max }(\AA)$ & 970 & 1100 & 1250 & 1520 & 1690 & 1870 \\
\hline
\end{tabular}

Tabela 3.1 - Propriedades médias de estrelas Be de B0 até B5 (Harmanec, 1988). São listadas a temperatura efetiva no polo, $T_{\mathrm{ef}}^{\text {polo }}$, a velocidade crítica, $v_{\text {crit }}$, a luminosidade, $L$, a massa, $M$, o raio equatorial, $R_{\text {eq }}$, e o comprimento de onda em que ocorre o máximo em emissão, $\lambda_{\max }$.

determinaram que a média da temperatura no disco é aproximadamente $60 \%$ da temperatura efetiva estelar, $T_{\text {ef }}$; portanto, para este nosso estudo adotamos a simplificação de que a escala de altura é fornecida pela equação 1.2 com temperatura de $0.6 T_{\text {ef }}$ e massa correspondentes a cada subtipo espectral estudado. Adotamos os parâmetros estelares de Harmanec (1988), o qual os determinou para diversos subtipos espectrais através da análise de estrelas B e de binárias visuais conhecidas. Estes parâmetros, listados na tabela 3.1, serão usados em toda a dissertação.

\subsection{Propriedades Termodinâmicas do Gás}

\subsubsection{A Distribuição de Temperatura}

Apresentamos, na figura 3.1(a), o comportamento radial da temperatura para os subtipos espectrais de B0 a B4 para uma densidade na base de $n_{0}=1.0 \times 10^{13} \mathrm{~cm}^{-3}$. O comportamento das curvas se assemelha, apresentando uma queda a um valor mínimo no intervalo $2 R_{\star}<r<4 R_{\star}$ e, depois, atingindo um patamar aproximadamente isotérmico entre $5 R_{\star}<r<8 R_{\star}$. Notamos, também, que o valor mínimo da temperatura ocorre a distâncias cada vez maiores, conforme avançamos os subtipos espectrais.

Na figura 3.1(b), comparamos a distribuição de temperatura para um mesmo subtipo espectral obtida para três densidades na base. As curvas, assim como as da figura 3.1(a), também apresentam uma queda na região mais interna, porém, é possível notar que o efeito de diminuição é maior para densidades na base maiores. Esta estrutura é discutida 


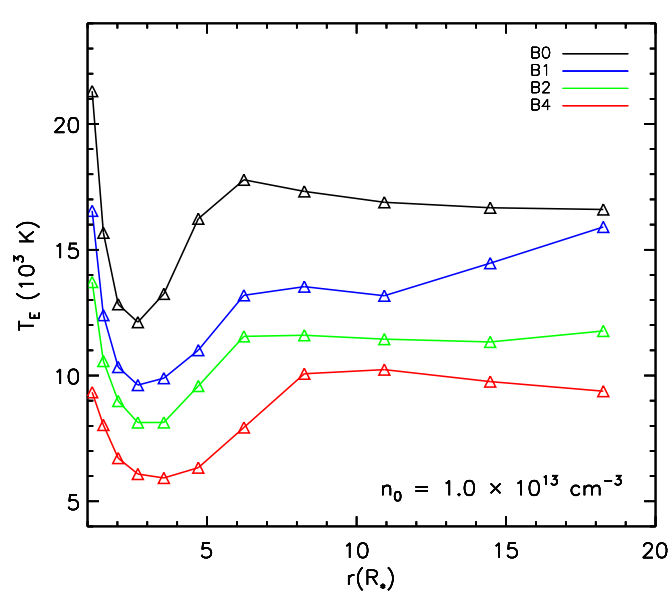

(a)

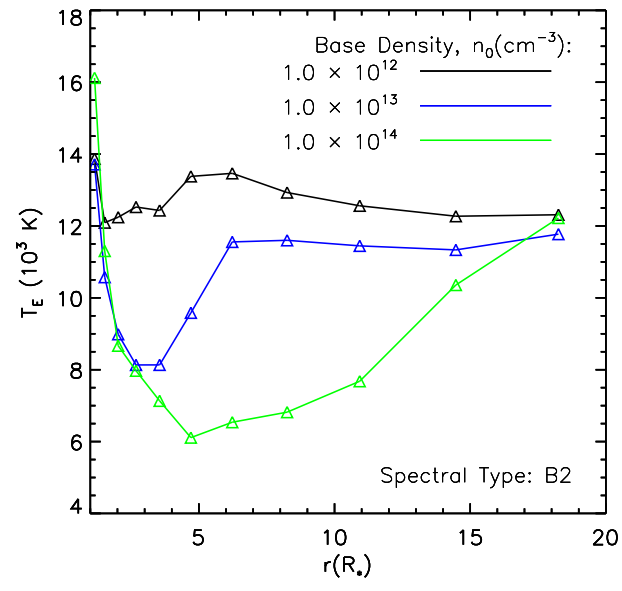

(b)

Figura 3.1: (a) Perfil de temperatura no plano equatorial do disco para os subtipos espectrais de B0 a B4 e (b) a mesma distribuição para o subtipo espectral B2 obtido para três densidades na base do disco.

por Carciofi e Bjorkman (2008), segundo os quais a parte não-isotérmica está associada à parte opticamente espessa mais interna do disco, a qual pode ser bem aproximada por um disco de reprocessamento infinitamente fino (Adams et al., 1987). A retomada da temperatura, após a rápida queda na parte interna, está associada ao fato do disco tornarse opticamente fino. No limite em que ele se torna muito opticamente fino, atingido nas regiões externas, o disco se torna aproximadamente isotérmico. Este efeito da transição do estado opticamente espesso para o opticamente fino pode ser visto na figura 3.1(b), em que o ponto de transição vai se tornando cada vez mais distante da estrela à medida que aumentamos a densidade.

Podemos usar a figura 3.1 para verificar nossa hipótese de que a escala de altura pode ser obtida tomando-se uma temperatura eletrônica de $0.6 T_{\text {ef }}$. Isto pode ser facilmente verificado através da parte externa de qualquer uma das curvas, onde o regime torna-se aproximadamente isotérmico. Vejamos, por exemplo, o subtipo espectral B0 que apresenta uma temperatura de $\sim 17000 \mathrm{~K}$ na região externa; comparando-a com a temperatura efetiva deste subtipo espectral mostrada na tabela 3.1, verificamos que $17000 \sim 0.57 T_{\text {ef }}$, o que valida nossa hipótese. 


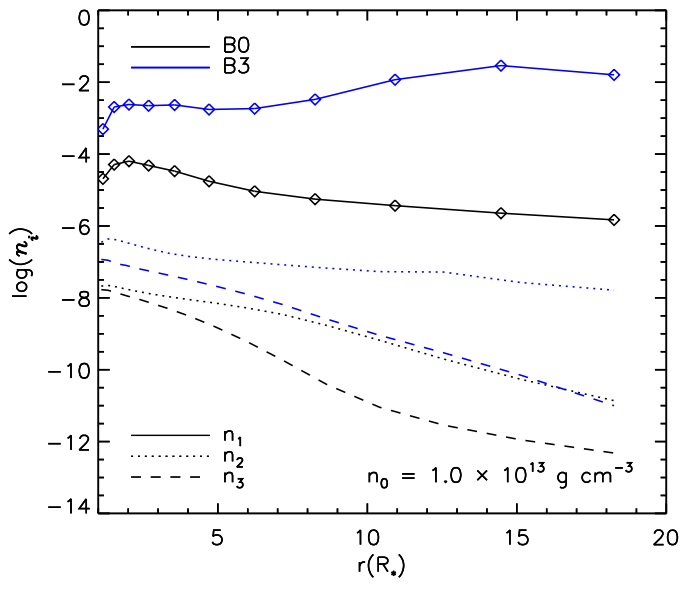

(a)

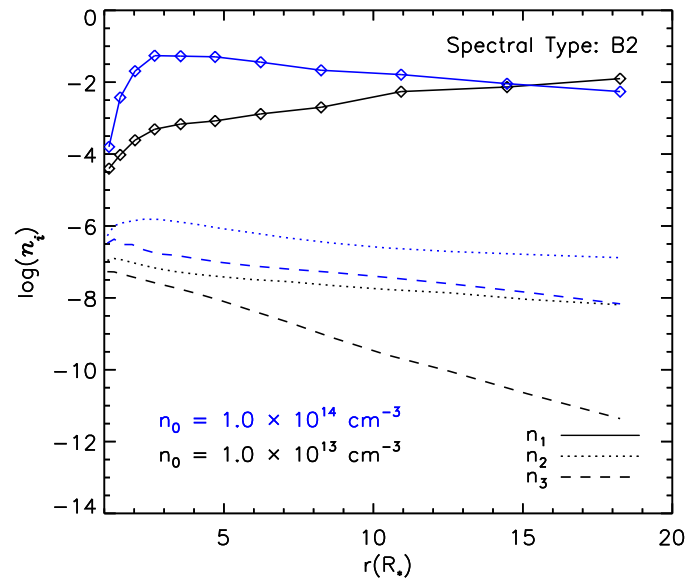

(b)

Figura 3.2: (a) Distribuição das populações dos níveis de 1 a 3 do hidrogênio ao longo do plano equatorial do disco para os subtipos espectrais B0 e B3. (b) A mesma distribuição para o subtipo espectral B2 com duas densidades na base diferentes.

\subsubsection{Populações do Hidrogênio}

Veremos que o espectro polarizado associado aos discos de estrelas Be está fortemente relacionado à absortividade do hidrogênio no disco. O código HDUst é o único existente capaz de calcular estas populações em sistemas tridimensionais, levando em conta todos os processos radiativos e colisionais existentes. Este estudo nos permitiu realizar uma comparação da população do H I com as populações dos outros níveis ao longo do plano equatorial do disco para os diferentes subtipos espectrais estudados.

Na figura 3.2(a) mostramos as populações dos níveis do hidrogênio para dois subtipos espectrais. É bastante evidente que para subtipos espectrais mais tardios as populações são relativamente maiores do que para os mais recentes. A explicação para isto tem a ver com o fluxo fotoionizante, pois quanto mais recente é o subtipo espectral de uma estrela maior é o fluxo na região do ultravioleta, que é o que controla as populações; portanto, é natural esperar que o disco em torno de uma estrela B0 seja muito mais ionizado do que o de uma estrela de subtipo espectral B3. Por exemplo, para a estrela B3 vemos que $\sim 1 \%$ dos átomos de hidrogênio estão no estado fundamental, caindo para $\sim 0.01 \%$ em uma B0. Veremos que esta forte dependência implicará em valores para a absortividade ligadolivre maiores para os subtipos espectrais mais tardios nas descontinuidades de Balmer e Paschen, influenciando fortemente o perfil do espectro polarizado (seção 3.5). 
Vejamos agora, as populações para diferentes densidades na base para um mesmo subtipo espectral (Fig. 3.2(b)). Verificamos que as populações nos três níveis mostrados é maior para a escala de densidade superior em praticamente todo o disco. Esta figura mostra que discos mais tênues possuem absortividade menor, ou seja, a baixas escalas de densidade, o fluxo fotoionizante é capaz de penetrar mais no disco, ionizando as suas

regiões mais centrais. À medida que aumentamos a densidade temos um efeito de shielding, em que as partes superiores do disco começam a bloquear a radiação fotoionizante, e que resulta no aumento das populações do hidrogênio na região equatorial.

Concluímos que tanto o subtipo espectral quanto a escala de densidade têm profundos efeitos na estrutura termodinâmica do disco, o que, como veremos, age modificando as diferentes absortividade e, consequentemente, o espectro polarizado.

\subsection{A Profundidade Óptica}

Um disco de Be tem três fontes principais de absortividade: eletrônica, ligado-livre e livre-livre. A primeira delas, a eletrônica, depende da quantidade de elétrons livres ou, de forma equivalente, à taxa de ionização; desta forma, um disco totalmente neutro não terá elétrons livres e, portanto, não terá absortividade de espalhamento eletrônico. Enquanto a absortividade de espalhamento tende a crescer com o nível de ionização, a absortividade ligado-livre, discutida em detalhes no apêndice C, apresentará comportamento contrário, aumentando com a diminuição do nível de ionização, basicamente por ser dependente das populações dos níveis do hidrogênio neutro. Desta maneira, a absortividade ligado-livre será nula no extremo de um disco completamente ionizado. Por fim, temos a absortividade livre-livre que tem a ver com processos colisionais entre elétrons e o campo de radiação, a qual também dependerá da taxa de ionização.

\subsubsection{A Profundidade Óptica e a Emissividade}

Para compreendermos melhor como a profundidade óptica se relaciona com a emissividade, voltemos à figura 1.5 na página 34. Comparando as curvas das fases ativa e pré-ativa notamos que o excesso de IV aumenta para comprimentos de onda maiores, ou seja, a absorção e a emissividade do disco aumentam com o comprimento de onda. Isto significa que, por exemplo, perto de $1 \mu \mathrm{m}$ ou de comprimentos de onda menores, a região emissora 
do disco é pequena, por causa do baixo valor da profundidade óptica ${ }^{2}$. Entretanto, para comprimentos de onda maiores, por exemplo, em $10 \mu \mathrm{m}$, uma região maior do disco passa a emitir, pois o aumento do comprimento de onda faz aumentar a profundidade óptica no disco, tornando maior o tamanho efetivo da região emissora.

Pelo fato do disco variar de tamanho ou densidade, seu crescimento também interfere na profundidade óptica. Por conseguinte, o excesso de fluxo, em um dado comprimento de onda, atinge um limite em que o tamanho da região emissora torna-se igual ao tamanho do disco. Neste ponto, o disco inteiro torna-se opticamente espesso e o excesso de IV não mais aumenta.

\subsubsection{A Profundidade Óptica Específica para Diferentes Escalas de Densidade}

Mostramos, na figura 3.3, a profundidade óptica total e a profundidade associada aos diferentes processos para duas escalas de densidade. As expressões utilizadas nesta determinação estão no apêndice C. Notamos, do modelo de baixa densidade $\left(n_{0}=\right.$ $1.0 \times 10^{12} \mathrm{~cm}^{-2}$ ), que a profundidade óptica total é basicamente plana, com exceção do salto de Lyman e do infravermelho mais distante. Como vimos na seção 3.3.2, isto se deve ao fato de discos de densidade mais baixa serem mais ionizados e, por conseguinte, terem a absortividade eletrônica como absortividade dominante. A situação muda quando vamos para densidades maiores, como mostrado na figura 3.2(b): à medida que aumentamos a densidade, aumentamos a fração de hidrogênio neutro, de forma que a absortividade dominante torna-se a ligado-livre. Em ambos os casos, a absortividade livre-livre é dominante no infravermelho, pelo fato de ser $\propto \lambda^{3} T^{-1 / 2}$ (ver Eq. C.1).

Podemos utilizar a figura 3.3 também para entender melhor o que ocorre na região de cada descontinuidade. Para isto, analisemos a região do salto de Balmer $(\lambda \simeq 3646 \AA)$, em que verificamos que fótons com comprimentos de onda superiores ao da descontinuidade, $\lambda>3646 \AA$, possuem energia suficiente para fotoionizar átomos de H I no estado $n=3$ e acima, o que significa que apenas átomos H I naqueles estados irão contribuir para a absortividade. Por outro lado, fótons com comprimentos de onda menores que o da des-

\footnotetext{
${ }^{2} \mathrm{~A}$ profundidade óptica e a emissividade são grandezas associadas, pois devem garantir a conservação do fluxo, ou seja, um disco que absorve pouco deve emitir pouco e vice-versa. A emissão pode ocorrer em comprimentos de onda diferentes dos fótons que são absorvidos.
} 


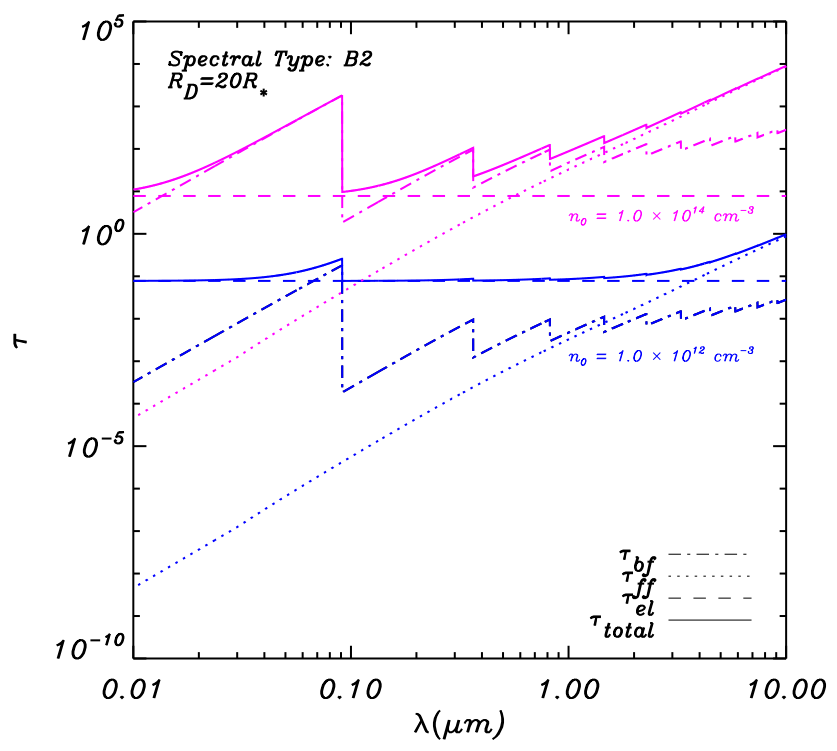

Figura 3.3: Profundidade óptica associada às diferentes absortividades, tomada em relação ao comprimento de onda para duas densidades na base do disco (azul: $10^{12}$ partículas por $\mathrm{cm}^{3}$, rosa: $10^{14}$ partículas por $\mathrm{cm}^{3}$ ). O livre-livre, o espalhamento eletrônico, o ligado-livre e a profundidade total são plotados em pontilhado, tracejado, ponto-tracejada e linha cheia, respectivamente.

continuidade, $\lambda<3646 \AA$, possuem energia para fotoionizar apenas a partir de $n=2$ em diante. Isto dá origem a um repentino crescimento na absortividade, o que, como veremos, está atrelado a uma forte queda no nível de polarização (ver seção 3.5). A mesma interpretação pode ser aplicada às demais descontinuidades.

\subsubsection{A Dependência Radial da Absortividade}

Agora que conhecemos a dependência espectral das absortividades, pode ser muito instrutivo entender onde elas são formadas no disco. Para isto, analisamos a dependência radial da absortividade para diferentes escalas de densidade. No apêndice C, mostramos que as diferentes absortividades $\alpha_{\mathrm{el}} \propto n_{0}\left(R_{\star} / r\right)^{3.5}, \alpha_{\mathrm{ff}} \propto n_{0}^{2}\left(R_{\star} / r\right)^{7}$ e $\alpha_{\mathrm{bf}} \propto \alpha_{0}\left(R_{\star} / r\right)^{3.5}$, onde $\alpha_{0}=\alpha_{0}\left(n_{0}, N_{2}, N_{3}\right)$, pois a absortividade ligado-livre depende das populações $N_{2}$ e $N_{3}$ do H I. Como estas populações variam com a distância à estrela (ver figura 3.2), espera-se que $\alpha_{0}$ possua uma dependência radial implícita. Isto implica que $\alpha_{\mathrm{bf}}$ terá uma dependência radial bem mais complexa que as demais absortividades. Assim, com o crescimento da densidade na base espera-se um crescimento linear do $\alpha_{\mathrm{el}}$, quadrático do $\alpha_{\mathrm{ff}}$ e aproximadamente quadrático do $\alpha_{\mathrm{bf}}$. 


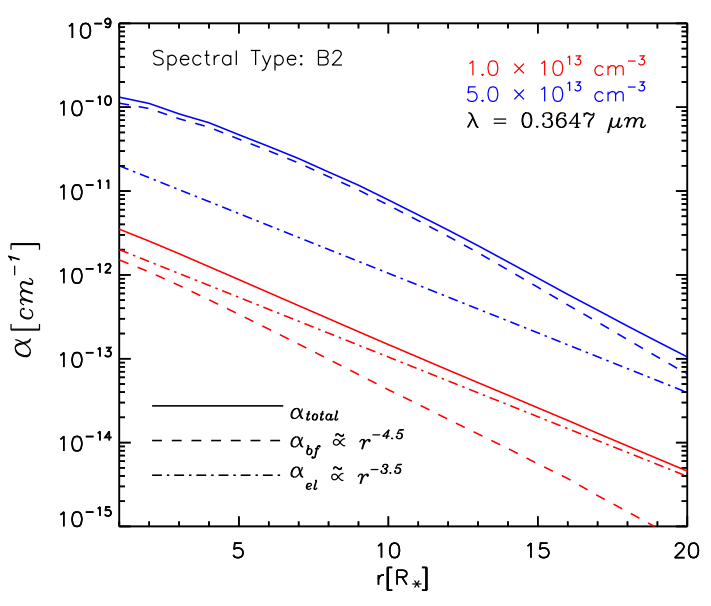

(a)

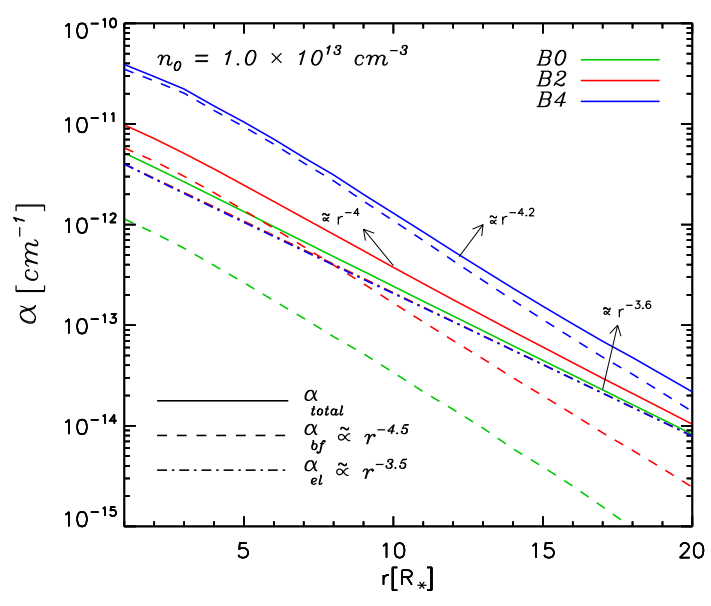

(b)

Figura 3.4: a) Variação radial das absortividades eletrônica e ligado-livre para o subtipo espectral B2 em dois regimes de densidade (vermelho: $1.0 \times 10^{13} \mathrm{~cm}^{-3}$ e azul: $5.0 \times 10^{13} \mathrm{~cm}^{-3}$ ). (b) O mesmo para outros três subtipos espectrais, B0, B2 e B4, para uma mesma densidade na base. São mostrados os ajustes radiais tomados para a região mais externa dos discos.

Na figura 3.4(a), mostramos como as diferentes absortividades variam radialmente para duas densidades na base. Note que na região espectral considerada a absortividade livrelivre é desprezível frente às demais. A figura mostra que a absortividade ligado-livre domina nas regiões mais internas do disco $\left(\lesssim 7.0 R_{\star}\right)$. Além disso, a figura 3.4(a) mostra que, enquanto a $\alpha_{\text {el }}$ cresce linearmente com $n_{0}$, como esperado, a absortividade ligado-livre cresceu um fator de 0.0004 a 0.01 , para $20 R_{\star}$ e de 0.14 a 3.5 para $1.01 R_{\star}$. Isso verifica a afirmação acima que a absortividade ligado-livre cresce aproximadamente com o quadrado da densidade.

Avaliamos também a dependência radial para diferentes subtipos espectrais. Mostramos na figura 3.4(b) uma comparação entre os subtipos espectrais B0, B2 e B4 para uma mesma escala de densidade e comprimento de onda. Nestas condições, a contribuição da absortividade eletrônica dos dois subtipos espectrais para a absortividade total é praticamente a mesma; entretanto, verificamos uma diferença acentuada entre os valores da absortividade ligado-livre. Como era esperado, a estrela de subtipo espectral mais recente apresenta valores menores para a absortividade ligado-livre, pelo fato de apresentar um fluxo fotoionizante maior, exibindo a absortividade eletrônica dominante em todo o disco. As curvas para o subtipo espectral B3 apresentam outro ponto interessante: verificamos, comparando-a com a figura 3.4(a), que a inversão de domínio das absortividades ocorre a 
distâncias progressivamente maiores da estrela à medida que consideramos tipos espectrais mais tardios. Em outras palavras, quanto mais fria a estrela maior será o volume do disco para o qual a absortividade ligado-livre é dominante.

As dependências radiais obtidas possuem relação direta com o perfil de densidade adotado para todas as absortividades estudadas. A absortividade ligado-livre, ao contrário das demais, possui forte dependência com as populações do hidrogênio neutro no estado 2 e 3 (ver apêndice C), mostrando ser mais influenciada pela população $N_{2}$, fazendo $\alpha_{\mathrm{bf}} \propto r^{-4.5}$ na região mais externa do disco.

\subsection{Características Polarimétricas de Discos em Estado Estacionário}

\subsubsection{Explicando a Forma do Espectro Polarizado}

Um espectro polarizado simples de ser explicado seria o de um disco de Be constituído apenas por elétrons livres. Neste caso, devido à independência da absortividade de espalhamento eletrônico com o comprimento de onda, teríamos um espectro polarizado plano. Um panorama mais realístico é obtido quando adicionamos átomos de hidrogênio neutro, H I, ao disco. A absortividade ligado-livre resulta em um padrão serrilhado no espectro polarizado (Fig. 1.3) que surge da relação entre a absorção pré e pós-espalhamento da luz estelar pelos átomos H I reduzir o fluxo polarizado e, portanto, o grau de polarização ${ }^{3}$. As alterações mais profundas no espectro polarizado ocorrem nos limiares de ionização do H I. Isto pode ser entendido através da figura 3.5, em que mostramos o espectro polarizado de um modelo de Be e a profundidade óptica associada às diferentes absortividades. Para o modelo de baixa densidade (Fig. 3.5(a)) o espectro é quase plano, porque é dominado pela absortividade eletrônica. Já para o modelo com densidade maior (Fig. 3.5(b)), o aspecto se torna serrilhado, por causa do domínio da absortividade ligado-livre sobre as demais.

A discussão anterior mostra que a forma detalhada do nível de polarização, no domínio do visível, dependerá fortemente das populações dos níveis $n=2,3$ e 4 do H I e de sua fração de ionização, a qual é responsável por fornecer o valor do número de elétrons contribuintes para o espalhamento eletrônico. Porém, por se tratar de um processo que depende de

\footnotetext{
${ }^{3} \mathrm{Um}$ detalhe importante a se acrescentar é que isto se deve a uma pequena fração de átomos neutros, variando de cerca de $0.1 \%$ nas regiões mais densas, próximas do plano médio, a ordens de magnitude menores nas outras partes (Carciofi e Bjorkman, 2006).
} 


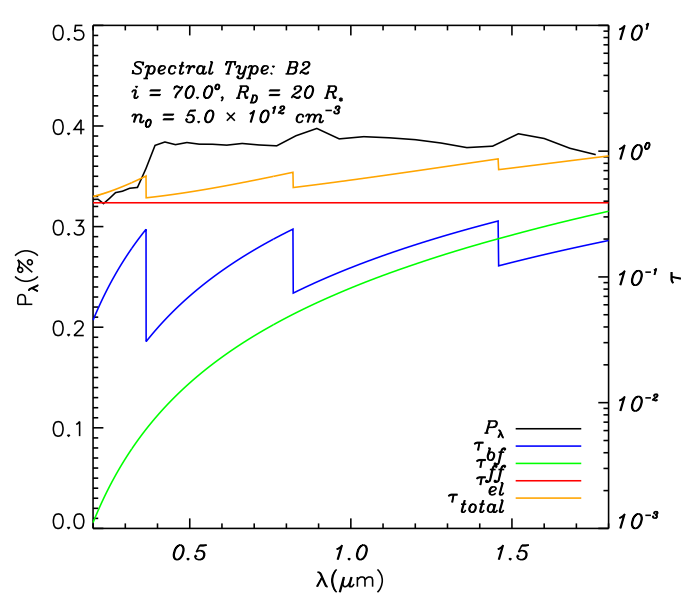

(a)

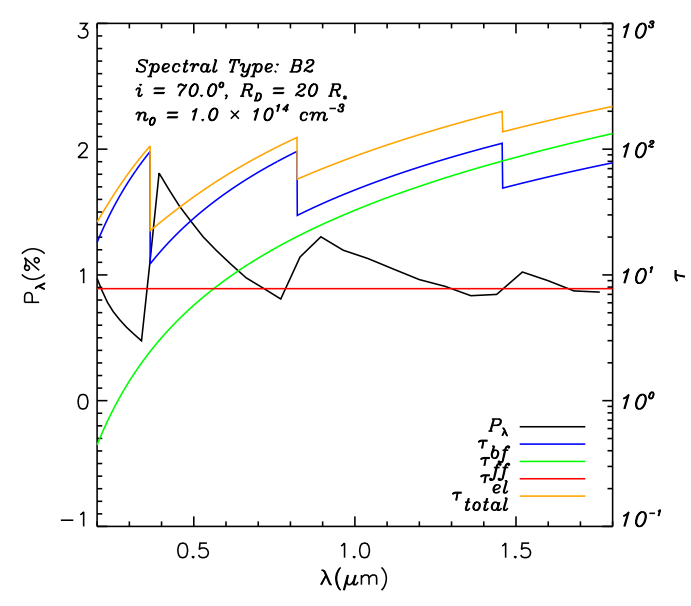

(b)

Figura 3.5: (a) Nível de polarização e a profundidade óptica associada às diferentes absortividades como função do comprimento de onda ao redor de uma B2 a um ângulo de inclinação de $70^{\circ}$ para um regime de baixa densidade e (b) para um regime de alta densidade (Haubois, Carciofi e Mota (2013), em preparação).

muitas variáveis discutimos, nas próximas seções, como o perfil do espectro polarizado se comporta conforme variamos alguns parâmetros.

Portanto, o aspecto serrilhado do espectro polarizado está ligado intrinsecamente à absortividade do H I. Na ausência de informações sobre a mudança da polarização no salto de Balmer, informações sobre a absortividade do H i podem ser obtidas de forma equivalente através da inclinação na região das descontinuidades do espectro polarizado. Como visto na figura 3.5, a inclinação aumenta com o crescimento da escala de densidade. Em analogia à SED, denominamos a inclinação do espectro polarizado de "cor". Para exemplificar, apresentamos a dependência com a escala de densidade na figura 3.6 para duas medidas, a razão das polarizações tomadas após e antes do salto de Balmer (apêndice B), $B J+/ B J-$, e a polarização na banda $V$. Constatamos, desta forma, que a polarização aumenta com a escala de densidade, porém, não tanto quanto o tamanho das descontinuidades; este aspecto explica o motivo do perfil serrilhado ser mais evidente para escalas de densidade maiores (Fig. 3.5).

Concluímos que o espectro polarizado possui duas características polarimétricas distintas: o nível de polarização, que depende do número de elétrons livres e sua geometria, e a "cor" do espectro polarizado (sua inclinação), a qual é uma indicadora da absortividade do H I. 


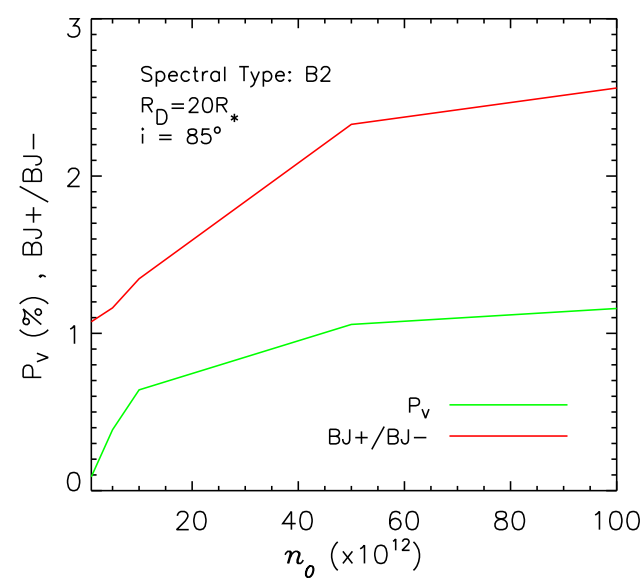

(a)

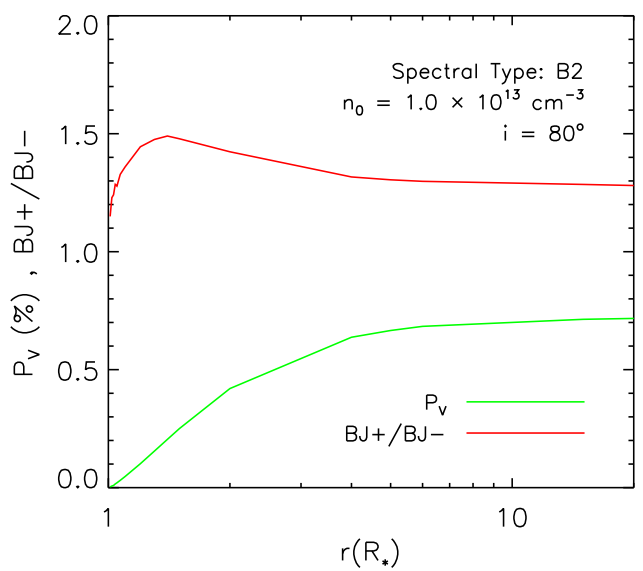

(b)

Figura 3.6: (a) Variação com a escala de densidade do disco da polarização na banda $V$, $P_{\mathrm{v}}$, e da razão $B J+/ B J-$. (b) Variação com o aumento do raio do disco, da polarização na banda $V$, $P_{\mathrm{v}}$, e da razão $B J+/ B J-$, para uma dada inclinação e densidade na base.

\subsubsection{Dependência do Espectro Polarizado com o Tamanho do Disco e a Escala de Densidade}

A figura 3.7(a) apresenta um exemplo de espectro polarizado simulado obtido para diferentes tamanhos de disco. A principal característica observada, na figura, é o aumento do nível de polarização com o crescimento do disco, resultado do aumento da massa de espalhadores (elétrons livres), como vimos nas seções anteriores.

Notamos que a partir de um dado tamanho de disco, $\sim 8 R_{\star}$, não há uma mudança significativa no nível de polarização, $P_{\lambda}$. Tal efeito pode ser compreendido calculando-se a massa total do disco como função do raio do mesmo. Para isto, podemos fazer a integral da massa utilizando a equação 1.1 para a densidade,

$$
M=\int_{0}^{2 \pi} \int_{\bar{r}_{\mathrm{i}}}^{\bar{r}_{\mathrm{e}}} \rho r^{2} d r d \phi=\frac{4}{3} \pi \rho_{0} R_{\star}\left(\frac{1}{\bar{r}_{\mathrm{i}}^{1.5}}-\frac{1}{\bar{r}_{\mathrm{e}}^{1.5}}\right),
$$

onde $\bar{r} \equiv r / R_{\star}, r_{\mathrm{i}}$ é o raio interno e $r_{\mathrm{e}}$ o raio externo. Com esta relação, calculamos a razão entre a massa de um disco de certo raio e a massa de um disco infinito, $M_{\mathrm{D}}$,

$$
M_{r_{\mathrm{i}} \rightarrow r_{\mathrm{e}}}=\left(\frac{\bar{r}_{\mathrm{i}}^{-1.5}-\bar{r}_{\mathrm{e}}^{-1.5}}{1-\bar{r}_{\mathrm{d}}^{-1.5}}\right) M_{\mathrm{D}}
$$

onde na expressão acima fizemos $\bar{r}_{\mathrm{i}}=1$. 


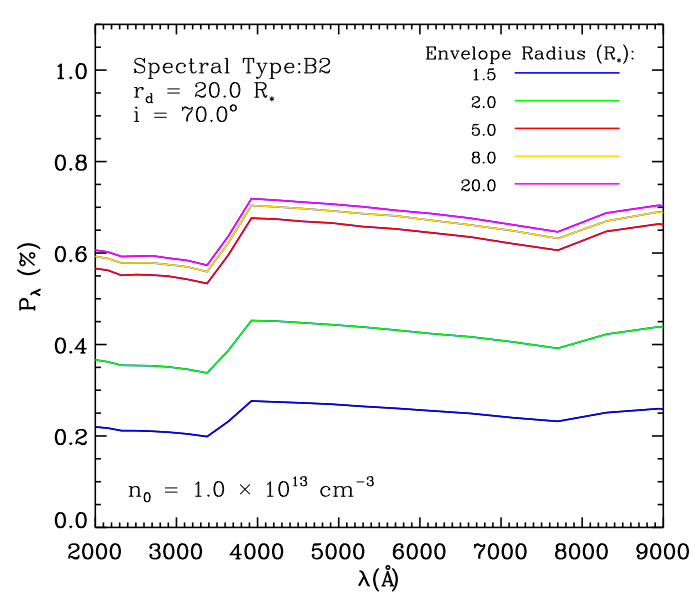

(a)

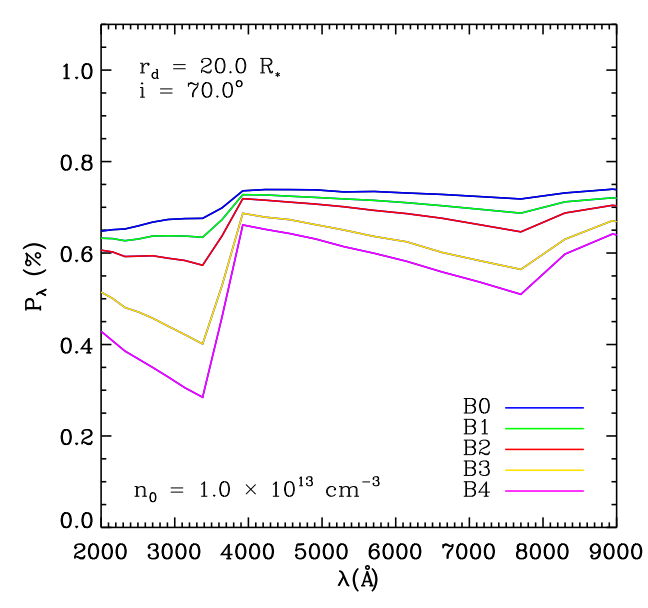

(b)

Figura 3.7: (a) Espectro polarizado de uma estrela B2 tomado para diferentes tamanhos de disco a uma densidade na base de $n_{0}=1.0 \times 10^{13} \mathrm{~cm}^{-3}$. (b) Espectro polarizado de um disco de $20 R_{\star}$ para diferentes subtipos espectrais, tomados para uma densidade na base de $n_{0}=1.0 \times 10^{13} \mathrm{~cm}^{-3}$.

Por exemplo, pode-se verificar que essa razão tem um valor de 0.97 para $\bar{r}_{\mathrm{e}}=8$. Desta forma, como a maior concentração de espalhadores se encontra na região mais interna, a maior parte da polarização deve se originar nesta mesma região.

Apesar do aumento do disco gerar um aumento da polarização, o mesmo não ocorre com o tamanho do salto de Balmer. Isso pode ser melhor constatado pela figura 3.6(b), em que mostramos o crescimento da razão $B J+/ B J-$, e da polarização na banda $V$ como função do raio externo do disco. Esta figura mostra uma característica interessante, qual seja o comportamento complementar da medida $B J+/ B J-$ e da polarização na banda $V$, cada uma destas características polarimétricas amostra regiões distintas do disco.

\subsubsection{Dependência do Espectro Polarizado com o Subtipo Espectral}

Outro fator que exerce grande influência sobre o espectro polarizado é o subtipo espectral da estrela. Na figura 3.7(b), mostramos como a forma do espectro varia do subtipo espectral B0 ao B4 fixando um mesmo tamanho e escala de densidade para o disco. Notamos que o tamanho das descontinuidades aumenta quando prosseguimos de subtipos espectrais mais recentes para os mais tardios. Este aumento está relacionado com a dependência da absortividade ligado-livre com as populações do hidrogênio neutro, as quais dependem fortemente do regime de temperatura (seção 3.4). 


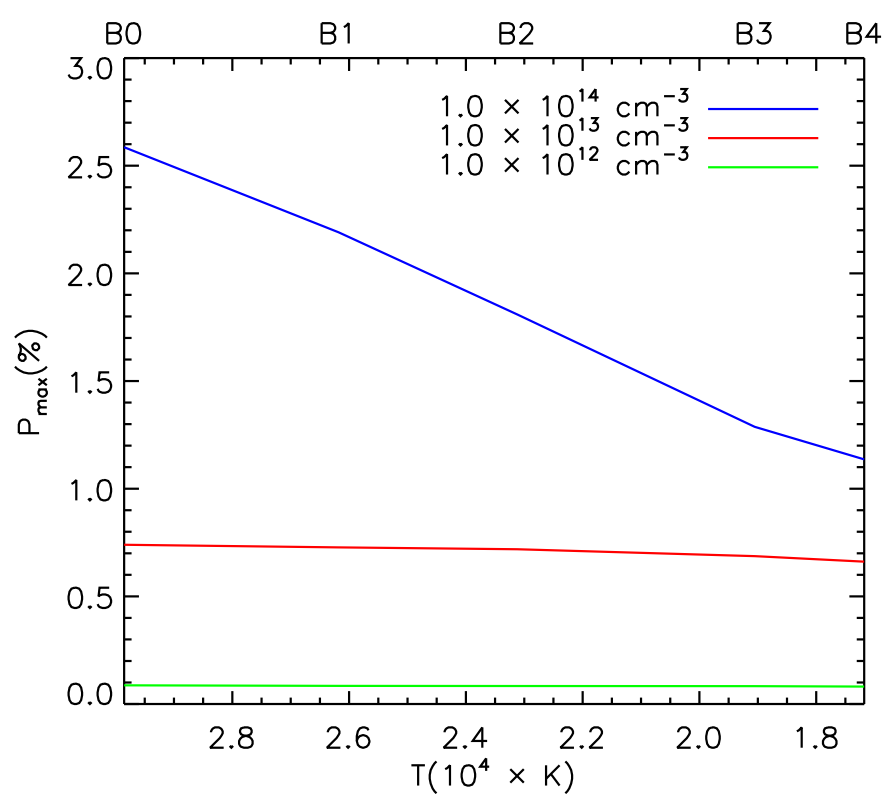

Figura 3.8: Nível máximo da polarização no visível de um disco com raio de $20 R_{\star}$ a $70^{\circ}$ versus a temperatura efetiva estelar para três escalas de densidade.

Na figura 3.8, mostramos o valor da polarização máxima atingida no visível para diferentes subtipos espectrais e escalas de densidade do disco. A curva verde mostra que a polarização máxima não varia significativamente com o subtipo espectral da estrela central para escalas de densidade muito baixas. Para densidades intermediárias (curva vermelha), notamos que o nível de polarização é levemente maior para subtipos espectrais mais recentes tendendo a decrescer conforme a temperatura diminui. Para densidades muito elevadas (curva azul), o efeito torna-se mais pronunciado, apresentando uma diferença de $1.5 \%$ entre a polarização máxima e a mínima. Estudos estatísticos que correlacionam a polarização intrínseca de estrelas Be com o subtipo espectral (e.g. Yudin, 2001) corroboram este resultado de que a polarização máxima cai fortemente com o subtipo espectral.

\subsubsection{Dependência do Espectro Polarizado com o Ângulo de Inclinação do Disco}

Mostramos, na figura 3.9, a mudança da polarização na banda $V$ com o ângulo de inclinação do disco para diferentes subtipos espectrais. Esta figura mostra que o máximo da polarização não ocorre para discos vistos de lado, mas quando $i \simeq 75^{\circ}$. Vimos, na seção 2.3.2, que efeitos como atenuação pré-espalhamento e espalhamento múltiplo tornam a dependência funcional de $P$ com $i$ mais complexa que a relação simples $P \propto \sin ^{2} i$ obtida 


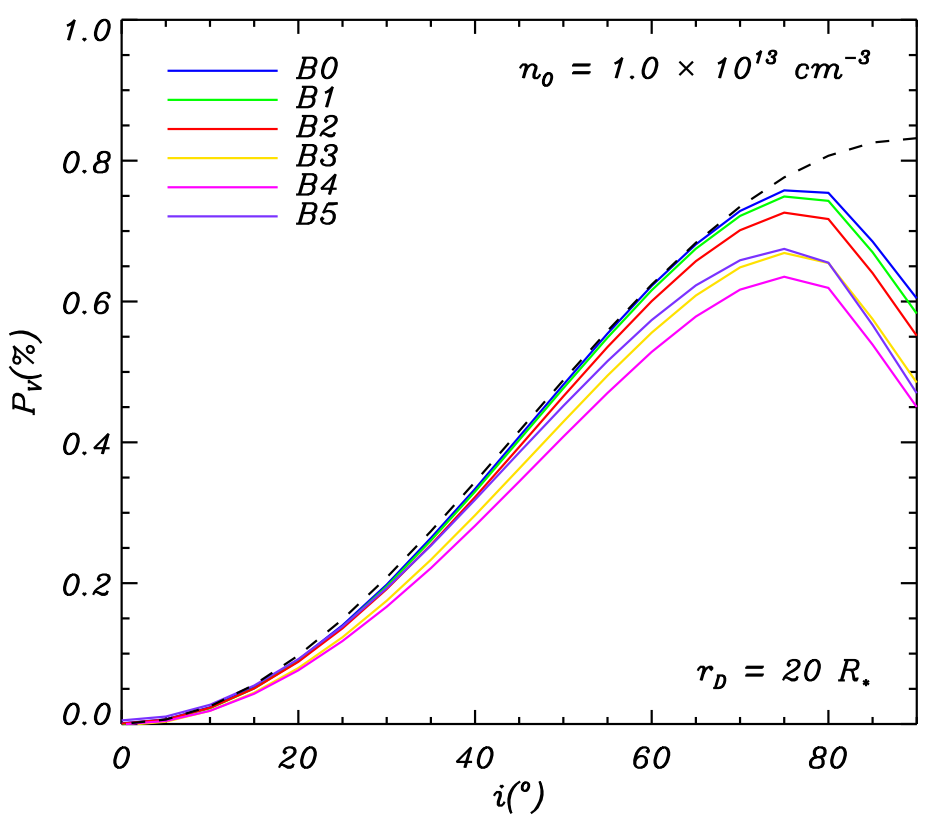

Figura 3.9: Mudança da polarização na banda $V$ com o ângulo de inclinação do disco, tomada para os subtipos espectrais de B0 a B5. A curva tracejada representa a curva teórica de Brown e McLean (1977).

por modelos de espalhamento simples (Brown e McLean, 1977; Wood et al., 1996a). Nossos resultados corroboram estudos anteriores e mostram a dependência funcional de $P$ com $i$ para o caso particular de estrelas Be.

A curva tracejada, na figura 3.9, representa o ajuste da contribuição do espalhamento eletrônico para um disco plano fornecido pela relação,

$$
P=P_{0} \sin ^{2}(i)
$$

onde $P_{0}$ foi determinado ajustando-se os dados para o modelo de B0. Como mostrado por Brown e McLean (1977), $P_{0}$ é uma função da profundidade óptica média $(\bar{\tau})$ e da geometria do envelope ( $\gamma$ na Eq. 2.21).

\subsection{Discussão}

Neste capítulo, apresentamos um estudo aprofundado das propriedades termodinâmicas e dos processos radiativos em discos estacionários de Be explorando, em particular, vários subtipos espectrais, diferentes escalas de densidade e tamanhos de disco. Isto possibilitou um estudo detalhado das características da profundidade óptica e do espectro polarizado, 
os quais foram avaliados com um olhar sobre a influência dos diferentes parâmetros do sistema. Parte desta análise é nova na literatura e alguns dos resultados apresentados neste capítulo farão parte de um artigo em preparação, feito em colaboração com o pósdoutor Xavier Haubois.

A análise das propriedades termodinâmicas e dos processos radiativos nos possibilitou entender o perfil de temperatura do disco, o qual, como vimos, possui dois regimes: um em sua região mais interna, em que a temperatura cai rapidamente, e outro na região mais externa, praticamente isotérmico. Em seguida, o estudo das populações do hidrogênio, realizado para diferentes escalas de densidade e subtipos espectrais, mostrou que as populações dos níveis do hidrogênio são altamente dependentes do subtipo espectral, sendo maiores para os subtipos mais tardios. Além disso, verificamos que o aumento da escala de densidade do disco diminui sua fração de ionização, fazendo a absortividade ligado-livre se tornar dominante em relação à eletrônica, ocasionando uma diminuição do nível de polarização e aumento da "cor" (inclinação) do espectro polarizado. Por fim, vimos que o conhecimento de como as populações do hidrogênio variam conforme modificamos as características físicas da fonte fornece a base para a compreensão da estrutura serrilhada do espectro polarizado. Explicamos esta estrutura através da importante contribuição do hidrogênio neutro na absortividade total.

Verificamos que o espectro polarizado possui duas características polarimétricas distintas: o nível de polarização, que depende da inclinação do disco e do número de elétrons livres, e a "cor" do espectro polarizado (sua inclinação), a qual é uma indicadora da absortividade do H i e do subtipo espectral da estrela Be. Outro resultado importante é que estas características polarimétricas são complementares, no sentido que amostram regiões distintas do disco, a "cor" do espectro polarizado depende mais das regiões mais internas enquanto que o nível da polarização amostra um volume maior do disco. 
Capítulo 4

\section{Uma Visão do Diagrama Cor-Polarização por meio de Modelos Dinâmicos Simplificados}

\subsection{Resumo do Capítulo}

Nosso objetivo final (capítulo 5) é estudar a relação entre a mudança da polarização através das descontinuidades de Balmer e Paschen e o nível de polarização na banda $V$, durante o processo de formação e dissipação de discos de Be. Como passo preliminar, adotamos modelos de crescimento e dissipação do disco simplificados, a fim de analisar como o espectro emergente, com ênfase no espectro polarizado, varia com o tempo.

Estruturamos o capítulo introduzindo o modelo utilizado (seção 4.2) e apresentamos (seção 4.3) o comportamento dos observáveis simulados para estes modelos. Após este estudo, apresentamos uma análise dos diagramas cor-polarização durante as fases de crescimento e dissipação, para diferentes parâmetros do disco. Finalizamos com uma breve discussão sobre os resultados principais na seção 4.4 .

\subsection{Modelos Dinâmicos Simplificados}

O comportamento hidrodinâmico de discos de Be será discutido em detalhes no próximo capítulo. Entretanto, adiantamos dois resultados importantes que servirão de base para desenvolvermos um modelo aproximado destes sistemas:

1. A formação de um novo disco em torno de uma estrela Be é feita a partir de matéria ejetada pela estrela que se difunde para fora por meio de torques viscosos (ver, por exemplo, figura 5.2(a)). Assim, podemos simular o crescimento do disco considerando 


\section{Crescimento}

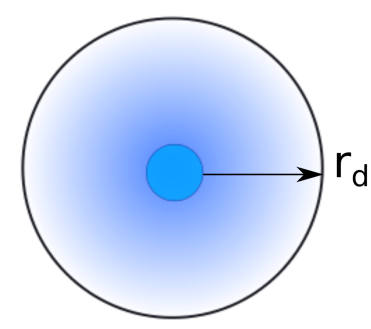

(a)

\section{Dissipação}

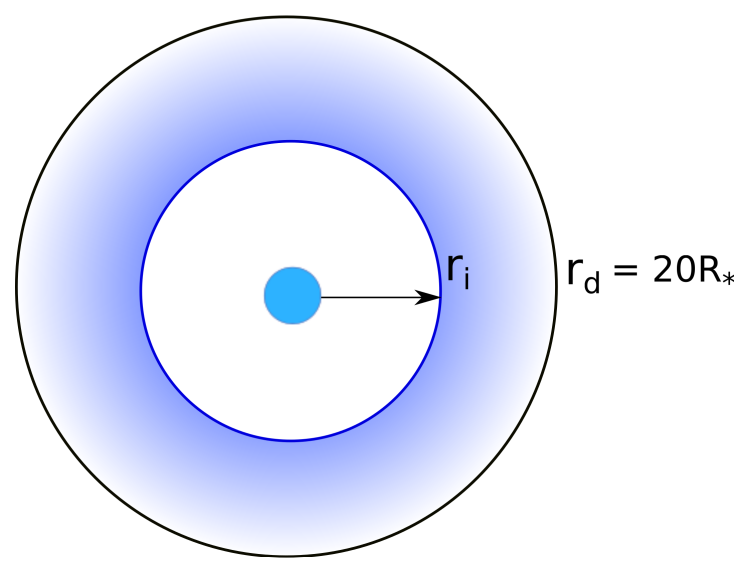

(b)

Figura 4.1: (a) Esquema de como simulamos o processo de crescimento do disco variando o tamanho do disco, $r_{\mathrm{d}}$, e (b) o de dissipação do disco, onde consideramos um disco cheio, $r_{\mathrm{d}}=20 R_{\star}$ e buracos internos, $r_{\mathrm{i}}$, progressivamente maiores.

simplesmente discos com raios externos cada vez maiores (Fig. 4.1(a)). Em nosso estudo, consideramos diferentes tamanhos de disco no intervalo entre 1.01 a $100 R_{\star}$. Esta aproximação não leva em conta o fato de que a inclinação do perfil de densidade do disco diminui com o passar do tempo;

2. A dissipação de um disco antigo procede através da reacreção da matéria do disco de volta à estrela (Fig. 5.2(b)). Dessa forma, a dissipação pode ser simulada tomandose buracos (anéis vazios) com tamanhos progressivamente maiores. Neste estudo, consideramos buracos internos de 1.01 a $15 R_{\star}$, feitos em um disco com raio externo de $20 R_{\star}$ (Fig. 4.1(b)). Novamente, esta aproximação ignora importantes mudanças no perfil de densidade durante a fase de dissipação.

O valor do raio máximo do disco foi fixado em $100 R_{\star}$, por não verificarmos mudanças significativas no nível da polarização a partir deste valor (Fig. 3.7(a); pág. 71). Exploramos, além disso, diferentes subtipos espectrais para a estrela B central (de B0 até B5) para os mesmos parâmetros livres apresentados na seção 3.2. 


\subsection{Crescimento e Dissipação}

\subsubsection{Fotometria}

Inicialmente, estendemos a figura 1.6 de Carciofi (2011), discutida na seção 1.4.1. Tomamos, para este propósito, o fluxo normalizado para diversas bandas e a polarização na banda $V$ (Fig. 4.2) desconsiderando o fluxo estelar. Desta maneira, consideramos apenas o fluxo proveniente do disco neste novo estudo. Com isso, foi possível verificar que bandas com comprimentos de onda maiores amostram regiões maiores do disco. Por exemplo, a curva roxa, referente ao fluxo em $60 \mu \mathrm{m}$, amostra uma região maior do disco $\left(r \gtrsim 30 R_{\star}\right)$, enquando que o fluxo no visível atinge seu máximo nas regiões mais internas $\left(r \lesssim 6 R_{\star}\right)$. Logo, o melhor indicador da taxa de injeção é a sua magnitute no visível, por ela ser muito sensível às vizinhanças da estrela, enquanto que bandas espectrais longas irão amostrar regiões maiores. De Carciofi e Bjorkman (2006), podemos, para um modelo típico de disco isotérmico de Be, estabelecer uma relação entre os raios efetivos relativos a diferentes comprimentos de onda,

$$
\frac{R_{\mathrm{ef}}\left(\lambda_{2}\right)}{R_{\mathrm{ef}}\left(\lambda_{1}\right)}=\left(\frac{\lambda_{2}}{\lambda_{1}}\right)^{0.41} .
$$

Por exemplo, tomando o comprimento de onda na banda $V, \lambda_{1}=0.55 \mu \mathrm{m}$, e em $60 \mu \mathrm{m}$, temos $R_{\mathrm{ef}}(60 \mu \mathrm{m}) / \mathrm{R}_{\mathrm{ef}}(0.55 \mu \mathrm{m}) \simeq 6.8$. Da figura 4.2 , notamos que o máximo na banda $V$ é atingido em $\sim 5 R_{\star}$ e em $60 \mu \mathrm{m}$ é atingido em $\sim 30 \mathrm{R}_{\star}$, $\operatorname{assim} R_{\mathrm{ef}}(60 \mu \mathrm{m}) / \mathrm{R}_{\mathrm{ef}}(0.55 \mu \mathrm{m}) \simeq 6$, o que valida nossa aproximação.

Estudamos, além disso, o comportamento destas curvas para diferentes escalas de densidade do disco. A figura 4.3(a) apresenta o resultado para um disco com uma densidade na base de $n_{0}=1.0 \times 10^{13} \mathrm{~cm}^{-3}$ e a figura $4.3(\mathrm{~b})$ para uma densidade maior, $n_{0}=1.0 \times 10^{14} \mathrm{~cm}^{-3}$. Confrontando as duas figuras, constatamos que a região de formação dos observáveis aumenta bastante com o crescimento da densidade.

\subsubsection{Diagramas Cor-Polarização}

Em estudo recente, Draper et al. (2011) mostraram, através da análise da polarização intrínseca de estrelas variáveis Be, a existência de uma relação entre a variação da polarização através do salto de Balmer e o nível de polarização na banda $V$ (Fig. 1.9). 


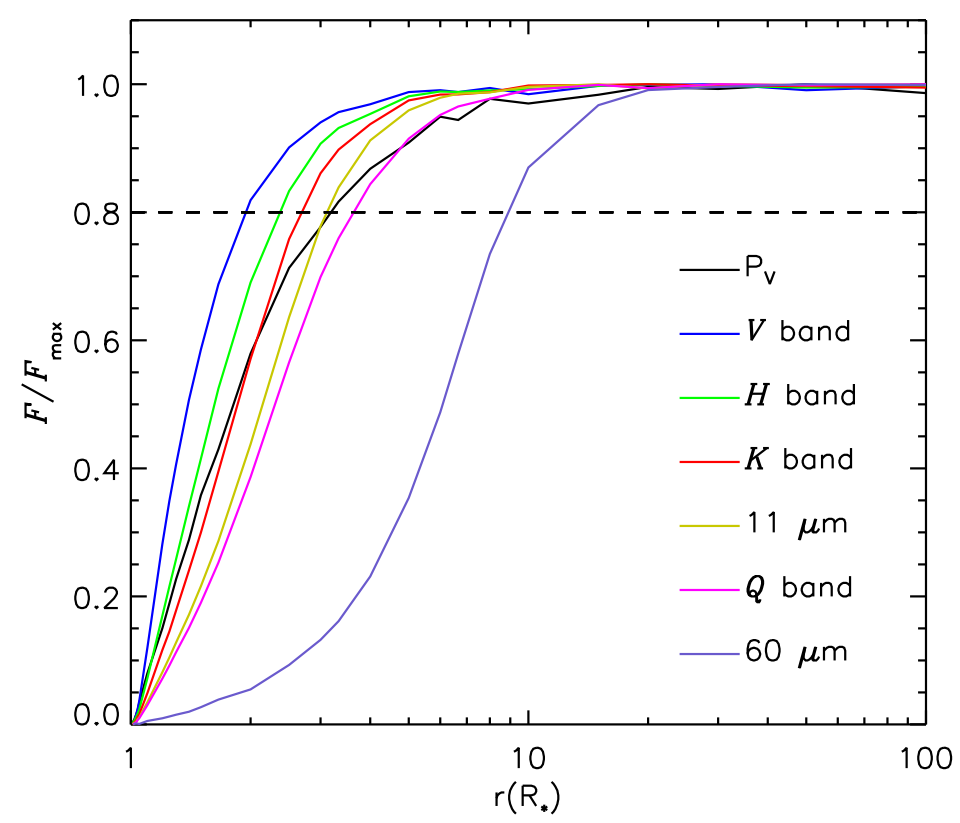

Figura 4.2: Fluxo normalizado tomado no contínuo para diversas bandas em diferentes regiões do disco de uma estrela de subtipo espectral B1 com densidade na base de $n_{0}=3.0 \times 10^{13} \mathrm{~cm}^{-3}$.

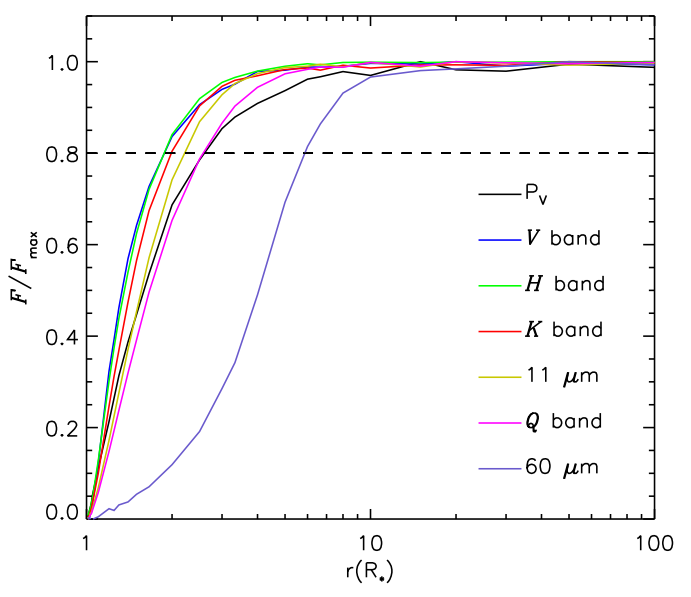

(a)

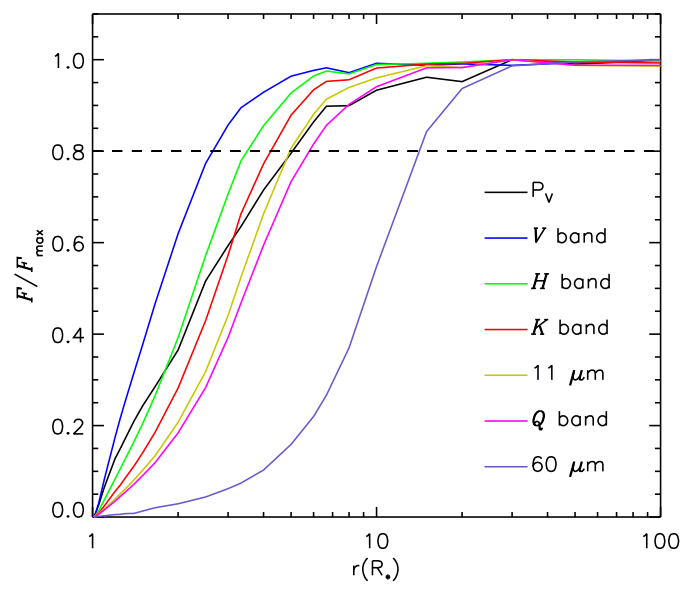

(b)

Figura 4.3: (a) Fluxo normalizado tomado no contínuo para diversas bandas em diferentes regiões do disco de uma estrela de subtipo espectral B1 com densidade na base de $n_{0}=1.0 \times 10^{13} \mathrm{~cm}^{-3} \mathrm{e}$ (b) o mesmo para a densidade na base de $n_{0}=1.0 \times 10^{14} \mathrm{~cm}^{-3}$. 


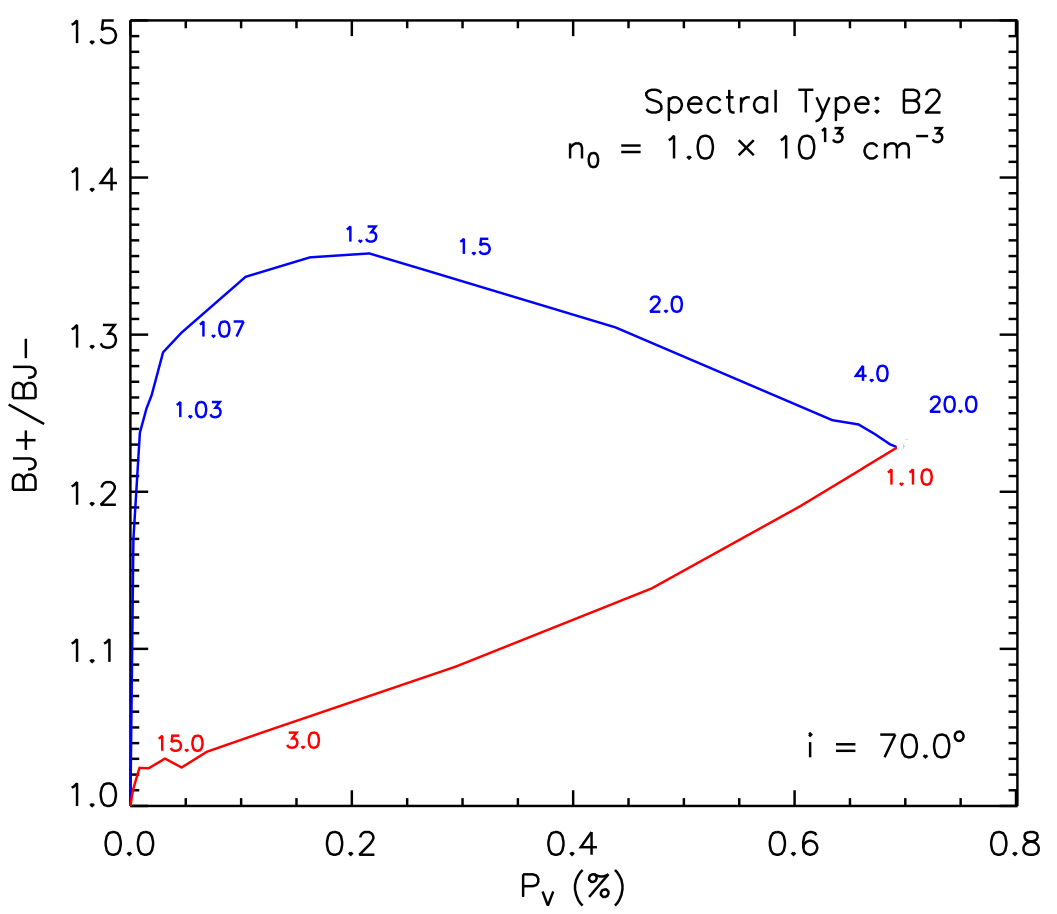

Figura 4.4: Diagrama cor-polarização para uma estrela de subtipo espectral B2. Os valores em azul representam os tamanhos do disco na fase de crescimento em unidades de raios estelares. Os valores em vermelho representam os tamanhos de alguns dos buracos internos, em unidades de raios estelares, que simularam a fase de dissipação.

Quando estas duas quantidades são graficadas uma versus a outra obtemos o diagrama cor-polarização (DCP); Draper et al. (2011) sugeriram que o processo de formação (crescimento) e dissipação do disco está associado a um loop no DCP.

Visando compreender a origem do loop no diagrama DCP e determinar qual o seu potencial de diagnóstico para inferir as propriedades físicas do disco circunstelar e/ou da estrela central, empreendemos um estudo sistemático do DCP usando os modelos simplificados descritos acima.

Na figura 4.4, mostramos um exemplo de DCP construído a partir dos modelos simplificados, para uma estrela B2. A parte superior do diagrama (em azul) representa o crescimento do disco. A razão $B J+/ B J-$, por ser uma medida direta da absortividade H I que é dominante nas partes mais internas (Fig 3.4) cresce muito rapidamente com o aumento do tamanho do disco. A forma detalhada desta curva depende das populações do nível 2 e 3 do H I, que controlam a profundidade óptica antes $\left(\tau^{-}\right)$e após $\left(\tau^{+}\right)$o salto de Balmer, respectivamente (apêndice B). O complexo comportamento das populações, associado ao fato do disco ser não isotérmico (cap. 3), faz que a razão $B J+/ B J-$ não 


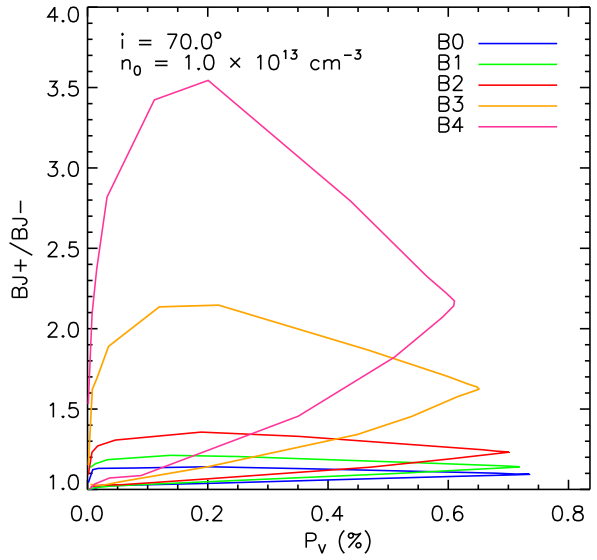

(a)

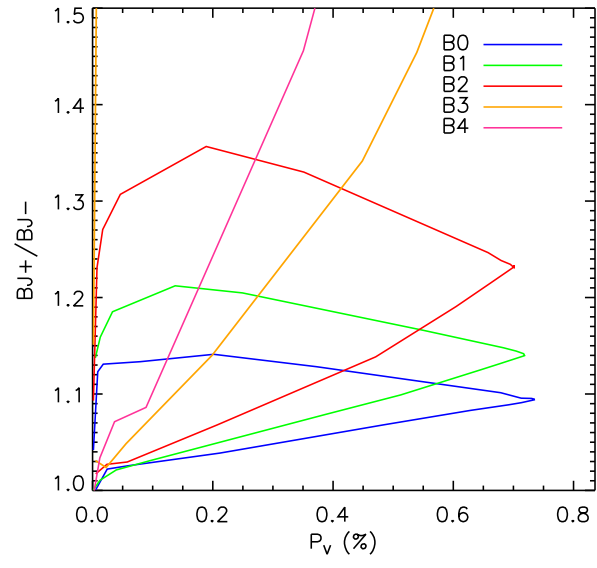

(b)

Figura 4.5: (a) Diagrama cor-polarização para cinco subtipos espectrais de B0 a B4. (b) Ampliação da região dos subtipos espectrais mais recentes $(\mathrm{B} 0 \rightarrow \mathrm{B} 2)$. Representamos em dois gráficos devido à diferença entre as escalas apresentadas entre os subtipos espectrais recentes e tardios.

seja monotonicamente crescente com o tamanho do disco. A abscissa do DCP (nível de polarização na banda $V$ ), ao contrário, cresce monotonicamente com $r_{\mathrm{d}}$ por depender da massa total de espalhadores.

A parte inferior do diagrama (em vermelho) representa a dissipação do disco, que, vale recordar, foi modelada tomando-se buracos progressivamente maiores em um disco "cheio" com $20 R_{\star}$. Neste caso, ambas as características polarimétricas estudadas (razão $B J+$ / $B J-$ e $\left.P_{\mathrm{V}}\right)$ decrescem monotonicamente com o aumento da cavidade interna. Entretanto, o ritmo de decrescimento é mais acelerado para o $B J+/ B J-$ do que para o $P_{\mathrm{V}}$. Novamente, este fato deve-se à predominância da absortividade ligado-livre nas regiões mais internas.

Nas próximas seções, continuamos nosso estudo do diagrama cor-polarização, estudando como sua forma é alterada conforme variamos diferentes parâmetros.

\section{Dependência do Diagrama Cor-Polarização com o Subtipo Espectral}

Das figuras 4.5, notamos um rápido crescimento do salto de Balmer quando vamos de subtipos espectrais mais recentes para os mais tardios, como resultado da crescente relevância da absortividade ligado-livre. Isto reflete no alargamento do loop de polarização, que para uma B0 torna-se muito estreito, tornando-se mais largo conforme avançamos para subtipos espectrais maiores. 


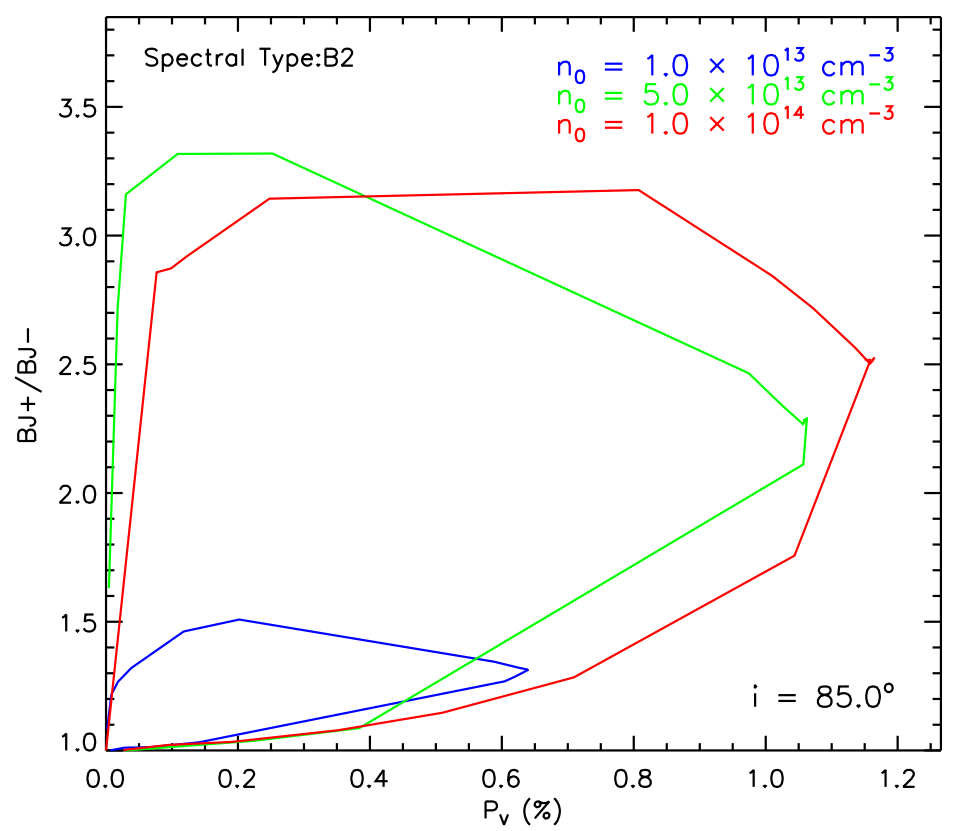

Figura 4.6: Diagrama cor-polarização de uma estrela de subtipo espectral B2 com diferentes densidades de base.

Outro aspecto interessante, apresentado na figura 4.5, é a diminuição da polarização máxima conforme aumentamos o subtipo espectral (ver Fig. 3.8; pág. 72). Neste caso, como a polarização é uma medida indireta da quantidade de elétrons livres no disco, se a absortividade ligado-livre é elevada, como em estrelas mais frias, a quantidade de elétrons disponíveis para a polarização será menor, fazendo a polarização diminuir.

\section{Diagrama Cor-Polarização em Diferentes Escalas de Densidade}

Testamos o comportamento do diagrama cor-polarização para diferentes densidades na base e um mesmo subtipo espectral (Fig. 4.6). Para compreender os resultados, vale a pena relembrar que a polarização tende a aumentar com o aumento do número de espalhadores no disco (seção 3.5.2), o que explica o crescimento observado na polarização máxima atingida quando aumentamos a escala de densidade do disco. Por outro lado, a razão $B J+/ B J$ - também cresce com o aumento da escala densidade, o que faz que a amplitude do loop aumente. 


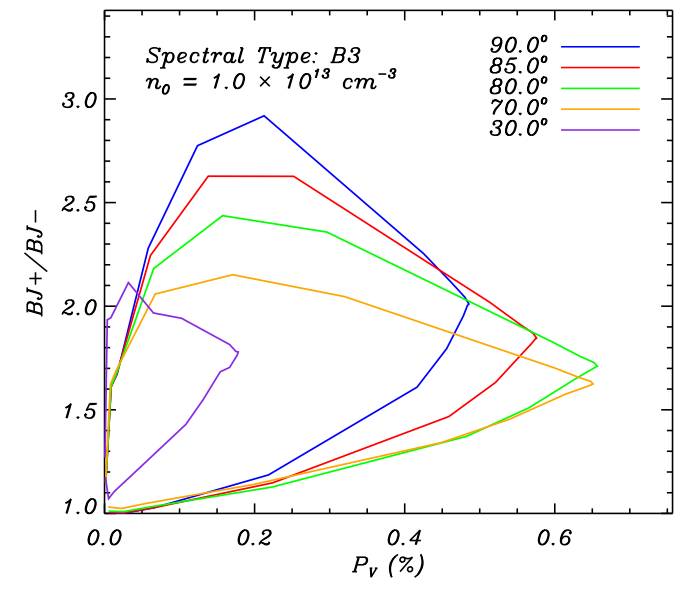

(a)

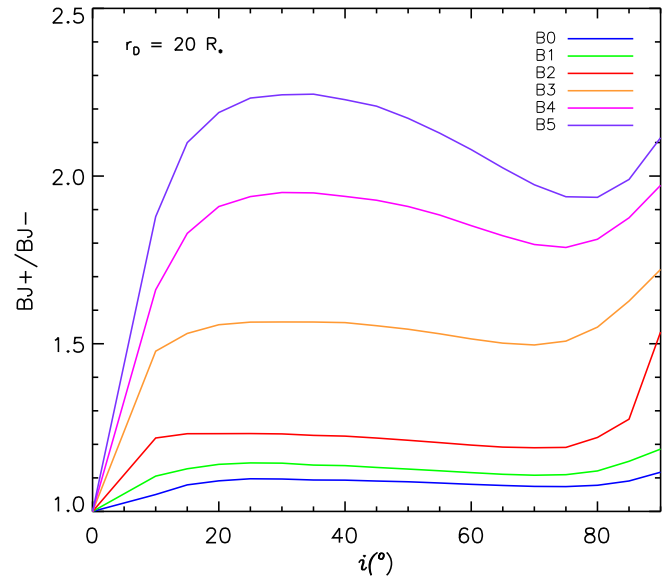

(b)

Figura 4.7: (a) Diagrama cor-polarização para uma estrela de subtipo espectral B3 tomado para diferentes ângulos de inclinação do disco, $i$. (b) Razão $B J+/ B J$ - tomada para diferentes subtipos espectrais por $i$.

\section{Dependência com o Ângulo de Inclinação do Disco}

O último parâmetro estudado foi o ângulo de inclinação do disco. Primeiro, para um subtipo espectral fixo, analisamos o diagrama cor-polarização visto de diferentes ângulos (Fig. 4.7(a)). Esta figura mostra que o tamanho do salto aumenta conforme nos aproximamos do plano do disco, enquanto que o valor da polarização máxima diminui. O aumento do tamanho do salto é explicado pelo fato da profundidade óptica aumentar conforme nos aproximamos das regiões mais densas no plano equatorial do disco. Com isso, a absortividade bound-free aumenta, tornando as descontinuidades maiores. Por outro lado, o comportamento da polarização deve-se ao aumento da auto-absorção quando nos aproximamos da configuração em edge-on (seção 3.5.4).

O gráfico da figura 4.7(b) mostra a razão $B J+/ B J$ - pelo valor do ângulo de inclinação do disco. Da figura, verificamos um aumento da razão para ângulos pequenos e uma diminuição em torno de $70^{\circ}$, que é justamente o ângulo para os quais a polarização apresenta valores mais elevados (Fig. 3.9). Estas duas dependências com $i$ explicam a complexa variação do loop do DCP com este parâmetro, apresentando uma relação complementar entre a variação da polarização numa descontinuidade em relação a polarização no contínuo. 


\subsection{Discussão}

Vimos, neste capítulo, que um modelo dinâmico simplificado para o crescimento e dissipação de um disco de Be produz um loop no diagrama cor-polarização como sugerido por Draper et al. (2011).

Mostramos que o processo de crescimento e dissipação de um disco Be pode ser detectado por via das duas características polarimétricas estudadas no capítulo 3: o nível da polarização no visível e a variação da polarização nas descontinuidades do hidrogênio. O estudo dependência da população do H I com os subtipos espectrais, realizado no capítulo 3 , tornou claro porque as descontinuidades se tornam maiores para subtipos espectrais B mais tardios, influenciando no aspecto do diagrama cor-polarização. Por exemplo, vimos que uma estrela de subtipo espectral mais recente possui temperatura suficientemente elevada para ionizar quase por completo o disco, tornando o salto de Balmer em polarização menos proeminente para estas estrelas.

O diagrama cor-polarização também foi analisado para diferentes escalas de densidade do disco e diferentes ângulos de inclinação, e mostramos que a forma do loop depende bastante destes dois parâmetros. Concluímos que o DCP tem um grande potencial diagnóstico. Enquanto que o tipo espectral pode ser facilmente obtido por meio da espectroscopia, o DCP pode potencialmente ser empregado para determinar o ângulo de inclinação e a escala de densidade do disco. 
Capítulo 5

\section{O Diagrama Cor-Polarização para o VDD Realista}

\subsection{Resumo do Capítulo}

Nos capítulos anteriores, analisamos o espectro polarizado de um VDD em estado estacionário (taxa de injeção de massa constante durante um longo período) e estudamos modelos dinâmicos simplificados visando explicar as propriedades observadas do diagrama cor-polarização. Neste capítulo, focamos no estudo da variabilidade de observáveis polarimétricos no contínuo, usando um ferramental teórico diferente do apresentado anteriormente, uma vez que consideraremos a evolução temporal das propriedades físicas do disco. A partir disto, mostramos que certas combinações de observáveis polarimétricos exibem características típicas de uma história de perda de massa, o que posiciona o DCP como uma ferramenta de diagnóstico para o entendimento da dinâmica de discos de Be. Iniciamos, apresentando o modelo dinâmico adotado e o método na seção 5.2. Na seção 5.3, apresentamos uma análise sobre as assinaturas dinâmicas na polarimetria e sobre as dependências do DCP. Na seção 5.4, apresentamos o potencial de diagnóstico do DCP e finalizamos, realizando uma discussão sobre os resultados principais obtidos na seção 5.5.

\subsection{Modelos Dinâmicos Realistas}

Predições de modelos mostram que o VDD constitui a teoria mais viável para se explicar os discos circunstelares de estrelas Be (Carciofi, 2011). Vimos apenas seu aspecto independente do tempo no capítulo 1. Entretanto, poucas são as estrelas para as quais tal aproximação é válida, pois a ampla maioria das estrelas Be exibe variabilidade em diferentes escalas temporais (ver seção 1.5). Recentemente, um modelo dependente do tempo 


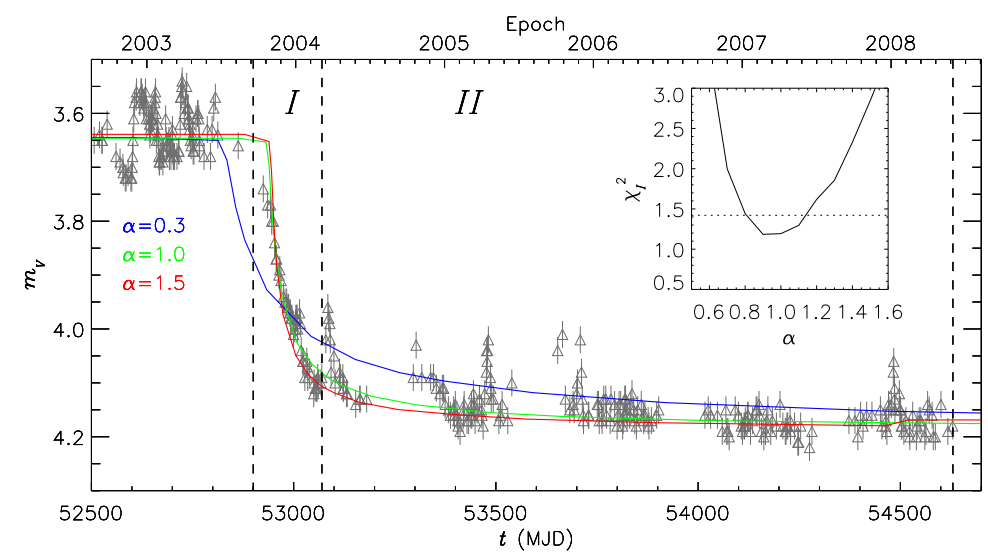

Figura 5.1: Curva de luz na banda $V$ da estrela 28 CMa. Os triângulos cinza correspondem às observações e as curvas representam os ajustes feitos para diferentes valores de $\alpha$ (Carciofi et al., 2012).

fois aplicado com sucesso na modelagem da dissipação do disco de 28 CMa (Fig. 5.1). Da análise, os autores determinaram o parâmetro de viscosidade do disco de uma Be, pela primeira vez, tendo encontrado $1.0 \pm 0.2$.

Nosso propósito é estudar o potencial de diagnóstico da polarização de um ponto de vista mais geral que inclua o estudo das consequências sobre a estrutura do disco e observações de sistemas com taxas variáveis de perda de massa. Este estudo foi iniciado por Haubois et al. (2012), o qual apresentou a primeira análise aprofundada das propriedades de um disco dinâmicamente variável, fornecendo a evolução de observáveis para diversos cenários dinâmicos e condições de inferir sobre as características físicas que regem a evolução dos discos de Be. Para tal, foram realizadas simulações das variações da estrutura de densidade superficial em um disco kepleriano isotérmico através do código SingleBe, o qual computa séries temporais de perfis de densidade para um dado cenário dinâmico.

\subsubsection{SingleBe}

O programa SingleBe (Okazaki et al., 2002) fornece o perfil unidimensional da densidade superficial de um disco isotérmico de decréscimo viscoso como função do tempo, resolvendo as equações hidrodinâmicas (Lynden-Bell e Pringle, 1974) numa aproximação de disco fino. A evolução de tal disco é descrita pela equação de difusão da densidade 
superficial (Pringle, 1981),

$$
\frac{\partial \Sigma}{\partial t}=\frac{1}{r} \frac{\partial}{\partial r}\left[\frac{\frac{\partial\left(r^{2} \Sigma \alpha c_{\mathrm{s}}^{2}\right)}{\partial r}}{\frac{\partial\left(r^{2} \Omega\right)}{\partial r}}\right],
$$

onde $\alpha$ é o parâmetro de viscosidade de Shakura-Sunyaev, $c_{\mathrm{s}}$ é a velocidade do som isotérmica e $\Omega$ é a frequência angular da rotação do disco. Nesta equação, tomamos a frequência angular da rotação do disco como kepleriana, i.e., $\Omega(r)=\Omega_{\mathrm{k}}=\left(G M_{\star} / r^{3}\right)^{1 / 2}$. As condições de contorno necessárias são explicadas em Haubois et al. (2012).

Para as simulações deste capítulo, foi simulado, por meio do SingleBE, uma fase longa com uma injeção de massa constante para produzir o estado estacionário inicial do disco. Ao final deste período, a dissipação do disco foi simulada, desligando-se a injeção de massa $(\dot{M}=0)$.

\subsubsection{A Evolução Temporal da Densidade Superficial}

Os resultados de Haubois et al. (2012) mostraram que as curvas de luz para diferentes comprimentos de onda são específicas de uma história de perda de massa $(\dot{M}(t))$, ângulo de inclinação do disco $(i)$ e parâmetro de viscosidade $(\alpha)$. Desta forma, as propriedades observacionais de discos de Be variáveis sugerem a existência de pelo menos duas escalas de tempo no controle das características fotométricas:

1. $\tau_{\text {in }}$, escala de tempo da variabilidade da injeção de massa no disco, relacionada com a duração e amplitude de eventos de ejeção de massa por parte da estrela;

2. $\tau_{\mathrm{d}}$, escala de tempo para o disco redistribuir o material injetado em sua base.

A evolução temporal de um dado sistema dependerá de uma interação complicada entre estas duas escalas de tempo. Mais especificamente,

1. $\tau_{\text {in }} \ll \tau_{\text {d }}$, nenhum efeito observável significante deverá ser produzido;

2. $\tau_{\text {in }} \gg \tau_{\mathrm{d}}$, podemos contemplar casos limite distintos: a criação de um novo disco alimentado a uma taxa constante de injeção de massa e a dissipação do disco préexistente depois que o fenômeno Be é desligado;

3. $\tau_{\text {in }} \sim \tau_{\mathrm{d}}$, haverá uma interação complexa entre as duas escalas de tempo. 
Haubois et al. (2012), estudando as condições 2 e 3, obteve os seguintes resultados:

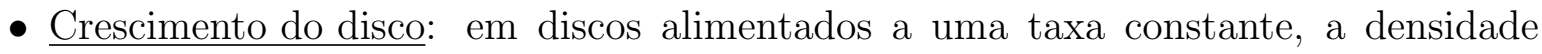
superficial, $\Sigma$, cresce com o tempo, variando fortemente com o raio (Eq. A.3). Nas regiões mais internas, o estado estacionário é atingido muito mais rápido do que na região externa;

- Dissipação do disco: quando o fenômeno Be é desligado em um disco completamente preenchido, a parte interna é reacretada e a externa é dissipada. Estas duas regiões são separadas por um ponto de estagnação, onde a velocidade radial é zero. Na região de dissipação, o expoente da lei de potência da densidade superficial é aproximadamente 2; na região de acreção, o expoente vai de 1.5, o qual é o valor para o disco de acreção em estado estacionário, para valores negativos próximos da estrela;

- Papel do $\alpha$ : nos casos limite mencionados acima, o $\alpha$ atua simplesmente modificando as escalas de tempo de crescimento e dissipação do disco. Desta forma, um disco com $\alpha=1$ cresce 10 vezes mais rápido que um com $\alpha=0.1$;

- Cenários periódicos: no caso 3, a densidade superficial pode ser uma função bastante complicada do raio e do tempo.

Mostramos exemplos dos resultados obtidos na figura 5.2, onde é destacada a evolução temporal da densidade superficial durante a fase de crescimento (painel esquerdo) e de dissipação (painel direito) para dois regimes de viscosidade. Durante a fase de crescimento, notamos que o disco leva um tempo maior para se aproximar do estado quasi-estacionário quando temos o parâmetro de viscosidade menor, ou seja, quando maior o $\alpha$ mais rápido será o crescimento do disco. Em relação à dissipação, notamos que o aumento do $\alpha$ faz o disco dissipar rapidamente. Por exemplo, para um período de 50 anos de dissipação $(\dot{M}=0$ por 50 anos) notamos que a escala de densidade na região interna do disco para $\alpha=1$ é aproximadamente duas vezes menor que a do sistema com $\alpha=0.1$. 

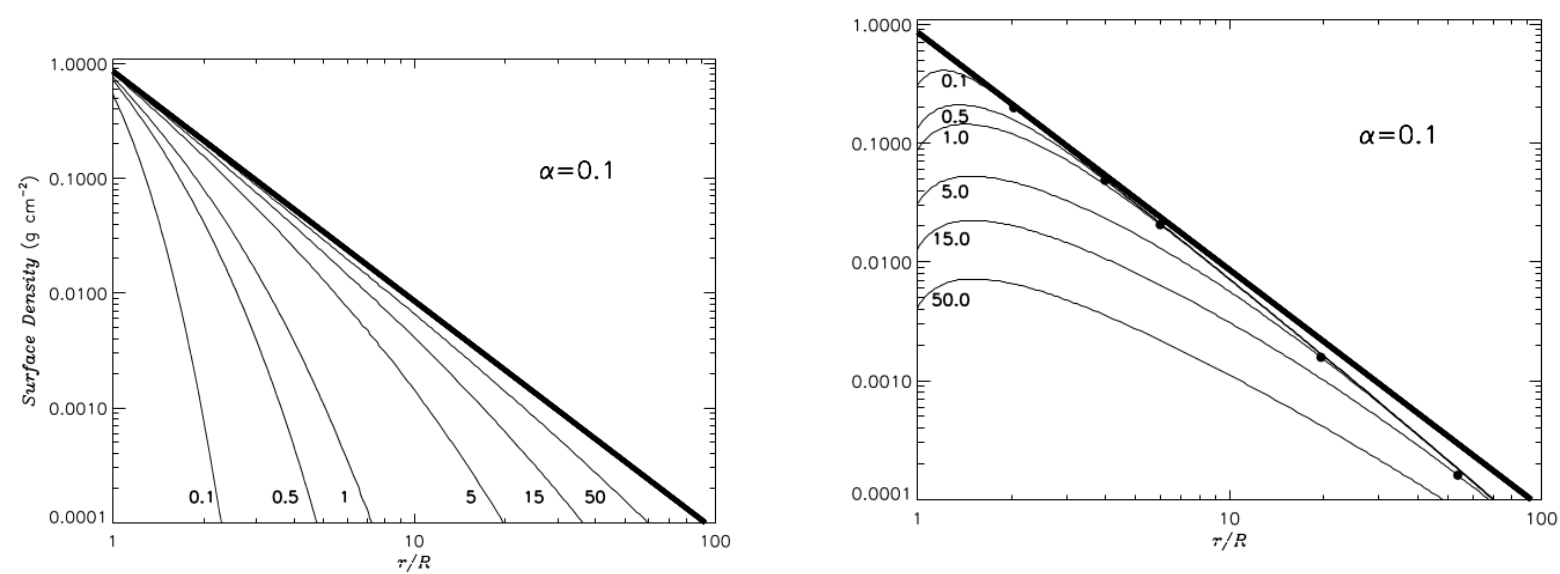

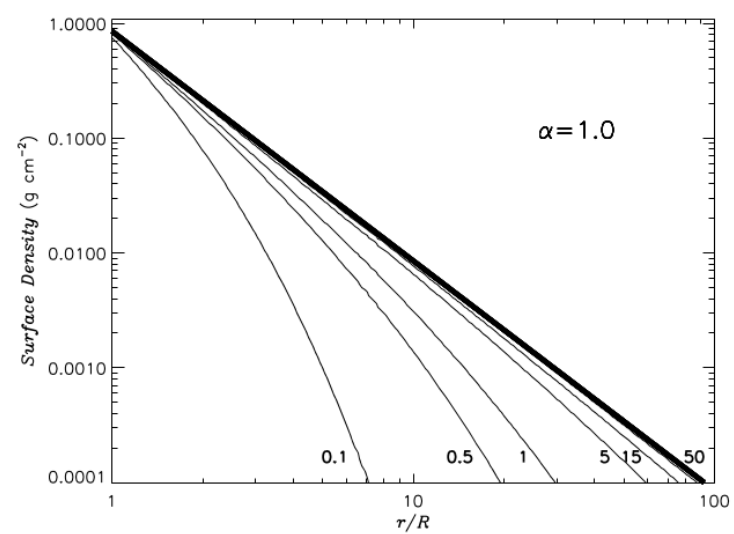

(a)

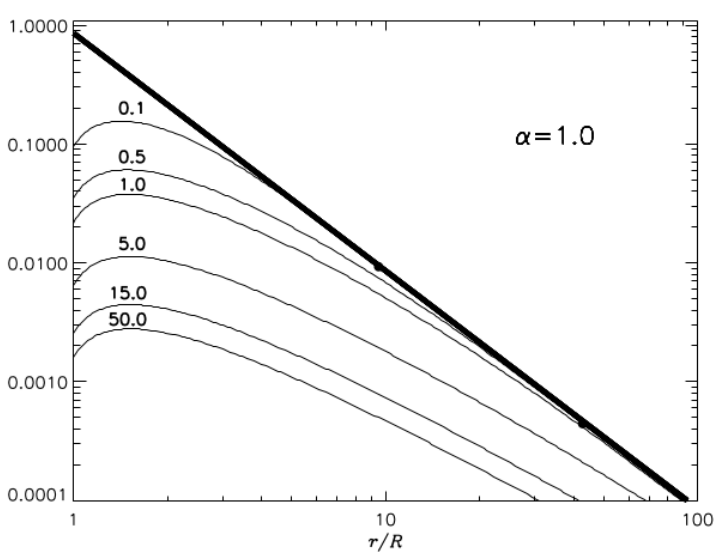

(b)

Figura 5.2: Evolução temporal da densidade superficial durante o crescimento (esquerda) e a dissipação (direita) do disco, para os parâmetros de viscosidade $\alpha=0.1$ e 1 . As épocas são contadas em anos e a linha grossa representa o estado estacionário do perfil de densidade. Os pontos pretos indicam o raio externo da região de acreção onde a velocidade radial é nula (ponto de estagnação) (Haubois et al., 2012).

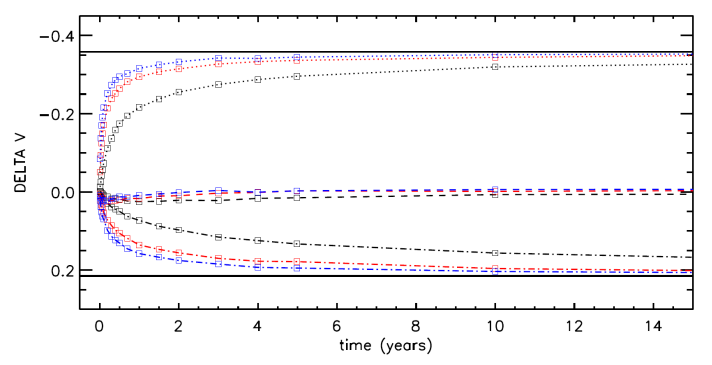

(a)

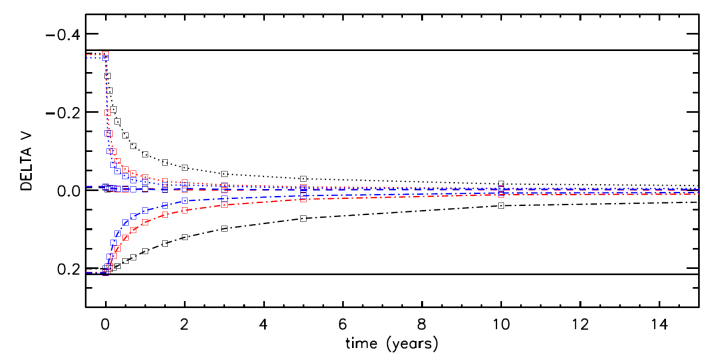

(b)

Figura 5.3: Curvas de luz na banda $V$ associadas com o crescimento do disco (painel superior) e dissipação (painel inferior). As linhas pontilhadas, tracejadas e ponto-tracejadas representam as curvas de luz para os ângulos de inclinação de $0^{\circ}$ (face-on), $70^{\circ}$ e $90^{\circ}$ (edge-on), respectivamente. As cores preta, vermelha e azul representam os modelos para $\alpha=0.1,0.5$, e 1.0, respectivamente. As linhas sólidas pretas indicam o valor assintótico de $\Delta V$ (Haubois et al., 2012). 


\subsection{Crescimento e Dissipação}

\subsubsection{Fotometria}

Mostramos, na figura 5.3, exemplos de curvas de luz na banda $V$ computadas para cenários dinâmicos de crescimento e dissipação estudados por Haubois et al. (2012). A figura mostra $\Delta V$, a diferença entre o fluxo total e o fluxo fotosférico na banda $V$. Esta figura mostra uma predição importante do VDD, a de que escalas de tempo para o crescimento do disco são menores que as escalas de tempo para a dissipação. Para entender esta diferença, é importante destacar que durante o crescimento do disco, as escalas de tempo são ajustadas pela redistribuição de matéria dentro de uma região muito pequena do disco. Durante a dissipação, as escalas de tempo são controladas pela reacreção por uma área muito maior do disco.

\subsubsection{Assinaturas Dinâmicas na Polarimetria}

Para entender os efeitos dos cenários dinâmicos sobre observáveis polarimétricos iniciamos com o caso de um crescimento e dissipação ininterruptos do disco, explorado na subseção anterior.

A figura 5.4 mostra a evolução temporal da polarização na banda $V, P_{\mathrm{V}}$, e a da polarização no salto de Balmer, $P_{\mathrm{BJ}}=B J+/ B J-$, para dois valores de $\alpha$ e três ângulos de inclinação. Em concordância com o mostrado na seção 4.3.2, o máximo do nível de polarização é atingido em valores próximos de $70^{\circ}$. Durante a fase de crescimento, notamos que $P_{\mathrm{BJ}}$ atinge seu máximo mais rápido do que $P_{\mathrm{V}}$. Quando a injeção de massa é interrompida, o disco interno é rapidamente reacretado pela estrela, isto causa a rápida queda do $P_{\mathrm{BJ}}$, mais sensível ao disco interno, e uma diminuição mais lenta de $P_{\mathrm{V}}$, por também ser sensível ao disco mais externo. Segue-se a dissipação secular de todo o disco, ao longo da qual $P_{\mathrm{BJ}}$ e $P_{\mathrm{V}}$ diminuem conforme a massa do disco decresce.

Os DCP obtidos dos cenários apresentados na figura 5.4 são apresentados na figura 5.5. Comparando-a com a figura 1.9 de Draper et al. (2012), notamos que um cenário simples de crescimento e dissipação é capaz de reproduzir qualitativamente as principais características observadas do DCP. 


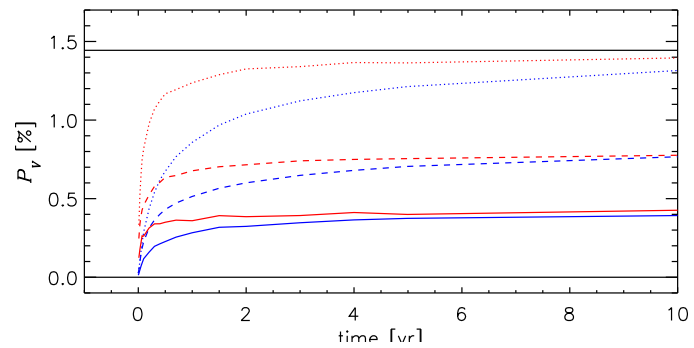

(a)

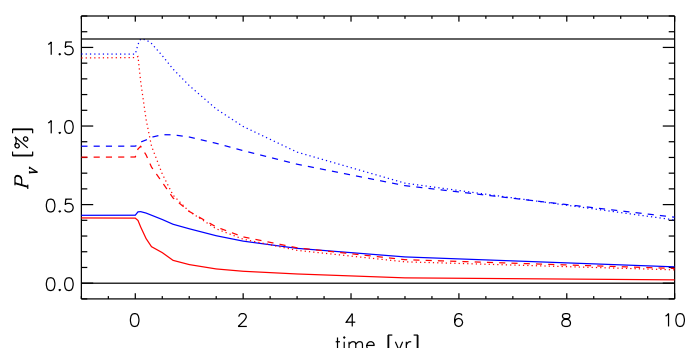

(c)

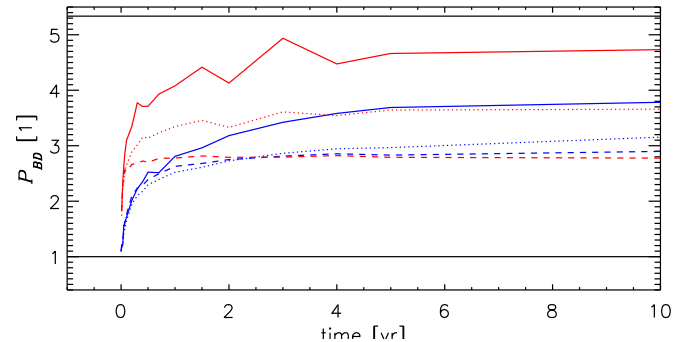

(b)

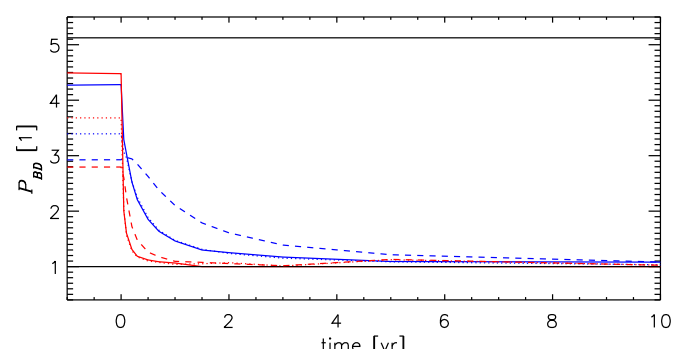

(d)

Figura 5.4: Evolução temporal de $P_{\mathrm{V}}$ (esquerda) and $P_{\mathrm{BJ}}$ (direita) associadas com o crescimento do disco (painel esquerdo) e dissipação (painel direito). As linhas sólida, pontilhada e tracejada representam as curvas de luz para os ângulos de inclinação de $30^{\circ}, 70^{\circ}$ e $90^{\circ}$, respectivamente. As curvas azuis e vermelhas representam os modelos para $\alpha=0.1$ e 1.0, respectivamente. As linhas pretas sólidas indicam os valores máximos para cada cenário.

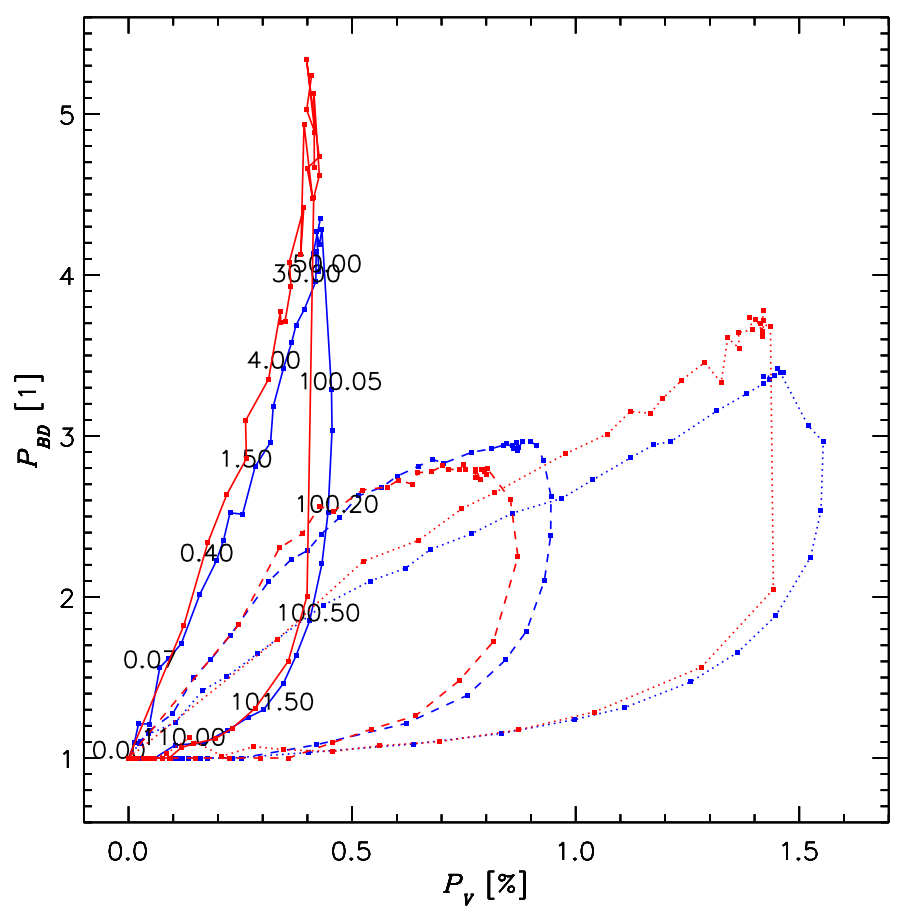

Figura 5.5: DCP em três ângulos de inclinação (linha sólida: $30^{\circ}$, pontilhada: $70^{\circ}$ e tracejada: $90^{\circ}$ ) para um cenário envolvendo uma fase de crescimento de 100 anos e uma de dissipação de 50 anos para dois parâmetros $\alpha$ (azul: 0.1; vermelho: 1.0). 


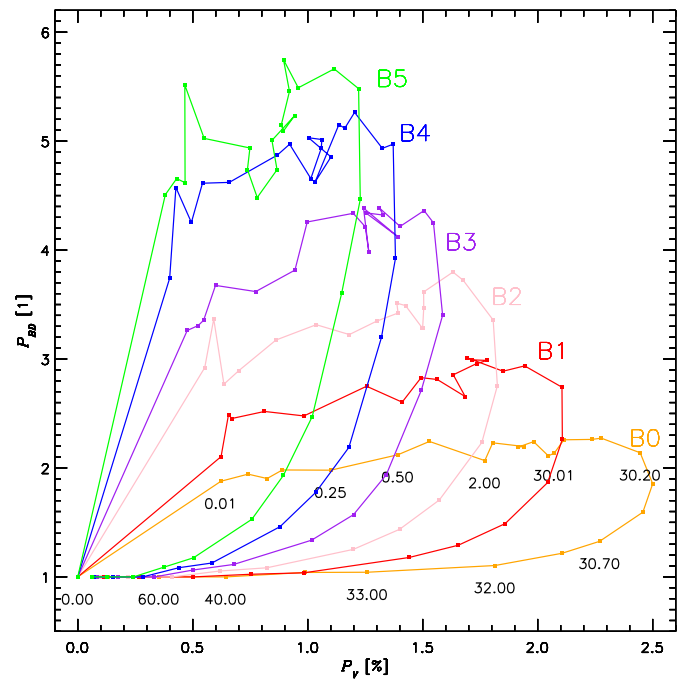

(a)

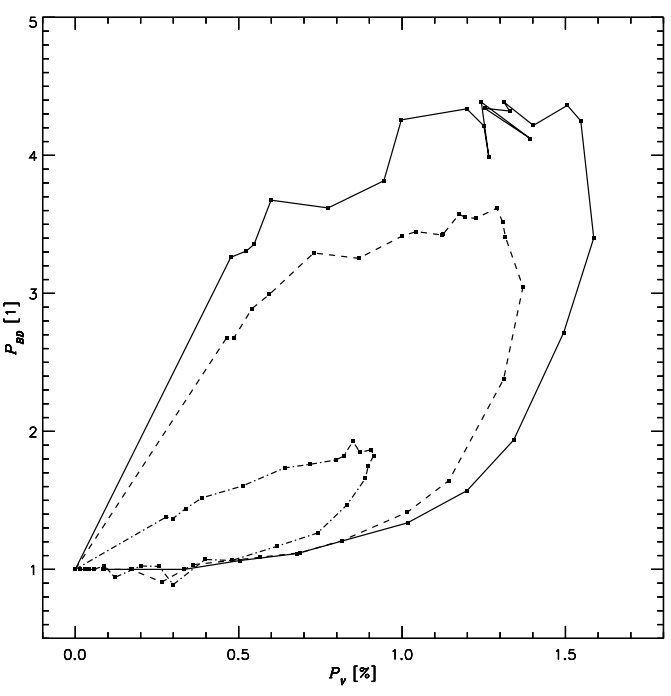

(b)

Figura 5.6: (a) DCP do $P_{\mathrm{BJ}}$ e $P_{\mathrm{V}}$ para seis subtipos espectrais distintos. A densidade na base é de $6 \times$ $10^{-11} \mathrm{~g} \mathrm{~cm}^{-3}$ e o ângulo de inclinação é de $85^{\circ}$. (b) DCP do $P_{\mathrm{BJ}}$ e $P_{\mathrm{V}}$ para três densidades: $6 \times 10^{-11} \mathrm{~g} \mathrm{~cm}^{-3}$ (linha sólida), $3 \times 10^{-11} \mathrm{~g} \mathrm{~cm}^{-3}$ (linha tracejada) e $1 \times 10^{-11} \mathrm{~g} \mathrm{~cm}^{-3}$ (linha ponto-tracejada). O cenário dinâmico envolve uma estrela de subtipo espectral B3 com uma fase de crescimento de 50 anos seguida de uma fase de dissipação de 50 anos. O ângulo de inclinação do disco é de $85^{\circ}$.

\section{Dependência do Diagrama Cor-Polarização com o Subtipo Espectral}

Mostramos, na figura 5.6(a), como o DCP dos modelos de crescimento e dissipação mostrados acima varia com o subtipo espectral da estrela central. Comparando-a com a figura 4.5, notamos que estes modelos reproduzem qualitativamente os resultados obtidos através dos modelos dinâmicos simplificados apresentados no capítulo 4, onde verificamos que a dependência com o subtipo espectral exerce maior influência sobre as descontinuidades ou, equivalentemente, à inclinação ou "cor" do DCP.

\section{O Diagrama Cor-Polarização em Diferentes Escalas de Densidade}

A figura 5.6(b) mostra como o DCP depende da densidade na base do disco. A amplitude do diagrama é claramente uma assinatura da densidade na base. Como mostrado previamente na figura 4.6, ocorre um aumento tanto de $P_{\mathrm{V}}$ quanto de $P_{\mathrm{BJ}}$, quando aumentamos a escala de densidade. Notamos, além disso, que a inclinação do DCP varia com a densidade. Para densidades baixas a inclinação é menor e o loop menos largo, enquanto que para densidades maiores a inclinação cresce e o loop torna-se mais largo. Este resultado é explicado pela influência da absortividade do H I em cada modelo. 


\section{Dependência do Diagrama Cor-Polarização com o Ângulo de Inclinação do Disco}

A figura 5.5 mostra que a trajetória no DCP possui forte dependência com o ângulo de inclinação do sistema. Na seção 4.3.2, verificamos um comportamento semelhante, cujos principais aspectos foram explorados por meio das figuras 3.9 e 4.7(b), as quais mostram o comportamento complexo de $P_{\mathrm{V}}$ e $P_{\mathrm{BJ}}$ com o ângulo de inclinação do disco.

\subsection{Potencial de Diagnóstico}

Na figura 5.6(a), podemos ver que o subtipo espectral impacta o DCP em vários aspectos. Primeiramente, o nível máximo atingido por $P_{\mathrm{V}}$ cresce com o aumento da temperatura. Segundo, a inclinação do DCP cresce com a diminuição da temperatura. Por outro lado, $P_{\mathrm{V}}$ atinge valores altos porque a baixa profundidade óptica permite o espalhamento sobre um largo volume do disco.

Portanto, efeitos semelhantes no DCP são causados tanto pela diminuição da temperatura (subtipos espectrais tardios) quanto pelo aumento da densidade do disco. Desta forma, concluímos que o potencial diagnóstico real do DCP depende criticamente de uma boa estimativa do subtipo espectral da estrela central.

\subsection{Discussão}

O fato dos observáveis apresentarem grande variabiliade posiciona o estudo dinâmico de estrelas Be como uma tarefa complexa, exigindo uma modelagem que alcance uma boa representatividade dos dados observados. Neste capítulo, discutimos o comportamento hidrodinâmico de discos de Be, investigando predições das características do espectro polarizado. Para isto, utilizamos uma modelagem mais realística baseada numa união de simulações de transferência radiativa (HDUST) e hidrodinâmicas (SINGLEBE). Esta combinação possibilitou mostrar como alguns observáveis polarimétricos evoluem com o tempo para cenários mais realistas de injeção de massa. Vimos, por exemplo, que o DCP associado à formação e dissipação do disco produz um loop que é morfologicamente muito semelhante às observações relatadas por Draper et al. (2011).

Concluímos, que os DCP constituem uma ferramenta muito poderosa de diagnóstico para a derivação de diferentes parâmetros físicos do disco, tais como o ângulo de inclinação, o co- 
eficiente de viscosidade $\alpha$ e a escala de densidade. Constatamos, além disso, aspectos novos, tais como a degenerescência, i.e., muitos cenários de perda de massa e parâmetros podem reproduzir igualmente o mesmo DCP observado. Os resultados e discussões apresentados nesse capítulo, acrescidos de estudos para cenários de perda de massa mais complexos, serão apresentados em um artigo que está sendo preparado em colaboração com o Dr. Xavier Haubois e o orientador e outros colaboradores (apêndice F). 


\section{Capítulo 6}

\section{Conclusões}

Este trabalho mostrou que um dos aspectos mais fascinantes de estrelas Be é a sua altíssima variabilidade que pode ocorrer em diferentes escalas de tempo. Salientamos o estudo dinâmico de estrelas Be que manifestam variabilidades de longo-período marcadas pela transição entre fases com e sem disco. Em particular, estudamos o Diagrama CorPolarização (DCP) inserido no contexto hidrodinâmico do Disco de Decréscimo Viscoso (VDD), atestamos que os diagramas teóricos reproduzem qualitativamente os diagramas observados. Mostramos, além disso, que o DCP pode ser uma ferramenta útil de diagnóstico das características físicas do disco, permitindo entender o comportamento das diferentes opacidades e de sua influência sobre o espectro polarizado, bem como o comportamento dinâmico do disco, todos avaliados com um olhar na influência gerada pelos diferentes parâmetros do sistema estrela-disco sobre os observáveis simulados.

Concluímos que o espectro polarizado possui duas características polarimétricas distintas: o nível de polarização, que depende da geometria do disco e do número de elétrons livres, e a "cor" do espectro polarizado (sua inclinação), a qual é uma indicadora da opacidade do H I e do subtipo espectral da estrela Be. Estas características polarimétricas são complementares no sentido de amostrarem regiões e aspectos distintos do disco.

Utilizar a polarimetria como ferramenta de diagnóstico do disco demonstra o valor de campanhas observacionais de monitoramento polarimétrico de longa duração, justificando a conservação das observações e de colaborações com outros grupos. Parte do trabalho apresentado é novo na literatura e alguns dos resultados serão apresentados em um artigo científico em colaboração com o Dr. Xavier Haubois e o supervisor. 


\section{Referências Bibliográficas}

Adams F. C., Lada C. J., Shu F. H., Spectral evolution of young stellar objects, ApJ, 1987, vol. 312 , p. 788

Bastian N., Covey K. R., Meyer M. R., A Universal Stellar Initial Mass Function? A Critical Look at Variations, ARA\&A, 2010, vol. 48, p. 339

Bjorkman J. E., Circumstellar Disks. In Stellar Atmospheres: Theory and Observations , vol. 497 of Lecture Notes in Physics, Berlin Springer Verlag, 1997, p. 239

Bjorkman J. E., Bjorkman K. S., The effects of gravity darkening on the ultraviolet continuum polarization produced by circumstellar disks, ApJ, 1994, vol. 436, p. 818

Bjorkman J. E., Carciofi A. C., NLTE Monte Carlo Models of the Polarization of Circumstellar Disks. In Astronomical Polarimetry: Current Status and Future Directions , vol. 343 of Astronomical Society of the Pacific Conference Series, 2005, p. 270

Brown J. C., McLean I. S., Polarisation by Thomson Scattering in Optically Thin Stellar Envelopes. I. Source Star at Centre of Axisymmetric Envelope, A\&A, 1977, vol. 57, p. 141

Carciofi A. C., The circumstellar discs of Be stars. In IAU Symposium, vol. 272 of IAU Symposium, 2011, p. 325

Carciofi A. C., Bjorkman J. E., Non-LTE Monte Carlo Radiative Transfer. I. The Thermal Properties of Keplerian Disks around Classical Be Stars, ApJ, 2006, vol. 639, p. 1081

Carciofi A. C., Bjorkman J. E., Non-LTE Monte Carlo Radiative Transfer. II. Nonisothermal Solutions for Viscous Keplerian Disks, ApJ, 2008, vol. 684, p. 1374 
Carciofi A. C., Bjorkman J. E., Otero S. A., Okazaki A. T., Štefl S., Rivinius T., Baade D., Haubois X., The First Determination of the Viscosity Parameter in the Circumstellar Disk of a Be Star, ApJ, 2012, vol. 744, p. L15

Carciofi A. C., Domiciano de Souza A., Magalhães A. M., Bjorkman J. E., Vakili F., On the Determination of the Rotational Oblateness of Achernar, ApJ, 2008, vol. 676, p. L41

Carciofi A. C., Miroshnichenko A. S., Kusakin A. V., Bjorkman J. E., Bjorkman K. S., Marang F., Kuratov K. S., García-Lario P., Calderón J. V. P., Fabregat J., Magalhães A. M., Properties of the $\delta$ Scorpii Circumstellar Disk from Continuum Modeling, ApJ, 2006, vol. 652 , p. 1617

Carciofi A. C., Okazaki A. T., Le Bouquin J.-B., Štefl S., Rivinius T., Baade D., Bjorkman J. E., Hummel C. A., Cyclic variability of the circumstellar disk of the Be star $\zeta$ Tauri. II. Testing the 2D global disk oscillation model, A\&A, 2009, vol. 504, p. 915

Clark J. S., Tarasov A. E., Panko E. A., Long term disc variability in the Be star $o$ Andromedae, A\&A, 2003, vol. 403, p. 239

Collins II G. W., Continuum Emission from a Rapidly Rotating Stellar Atmosphere., ApJ, 1963, vol. 138, p. 1134

Collins II G. W., The use of terms and definitions in the study of Be stars. In IAU Colloq. 92: Physics of Be Stars , 1987, p. 3

Cranmer S. R., A Statistical Study of Threshold Rotation Rates for the Formation of Disks around Be Stars, ApJ, 2005, vol. 634, p. 585

Curtiss R. H., Studies of class B stellar spectra containing emission lines. The spectrum of gamma Cassiopeiae.a, Publications of Michigan Observatory, 1916, vol. 2, p. 1

de Wit W. J., Lamers H. J. G. L. M., Marquette J. B., Beaulieu J. P., The remarkable light and colour variability of Small Magellanic Cloud Be stars, A\&A, 2006, vol. 456, p. 1027

Domiciano de Souza A., Hadjara M., Vakili F., Bendjoya P., Millour F., Abe L., Carciofi A. C., Faes D. M., Kervella P., Lagarde S., Marconi A., Monin J.-L., Niccolini G., Petrov 
R. G., Weigelt G., Interferometry of $\alpha$ Eri (Domiciano de Souza+, 2012), VizieR Online Data Catalog, 2012, vol. 354, p. 59130

Domiciano de Souza A., Kervella P., Jankov S., Abe L., Vakili F., di Folco E., Paresce F., The spinning-top Be star Achernar from VLTI-VINCI, A\&A, 2003, vol. 407, p. L47

Draper Z. H., Wisniewski J. P., Bjorkman K., Haubois X., Carciofi A. C., Bjorkman J. E., Meade M. R., Applying a New Polarization-based Diagnostic to an Existing Survey of Classical Be Stars. In American Astronomical Society Meeting Abstracts \#219, vol. 219 of American Astronomical Society Meeting Abstracts, 2012, p. 344.19

Draper Z. H., Wisniewski J. P., Bjorkman K. S., Haubois X., Carciofi A. C., Bjorkman J. E., Meade M. R., Disk-Loss and Disk Renewal Phases in Classical Be Stars III. Modeling Effects of Disk Structure. In American Astronomical Society Meeting Abstracts \#217 , vol. 43 of Bulletin of the American Astronomical Society, 2011, p. 339.22

Draper Z. H., Wisniewski J. P., Bjorkman K. S., Haubois X., Carciofi A. C., Bjorkman J. E., Meade M. R., Okazaki A., A New Diagnostic of the Radial Density Structure of be Disks, ApJ, 2011, vol. 728, p. L40

Eckart A., Schödel R., Meyer L., Trippe S., Ott T., Genzel R., Polarimetry of near-infrared flares from Sagittarius A*, A\&A, 2006, vol. 455, p. 1

Eisberg R., Resnick R., Quantum Physics of Atoms, Molecules, Solids, Nuclei, and Particles, 2nd Edition, 1985

Ekström S., Georgy C., Eggenberger P., Meynet G., Mowlavi N., Wyttenbach A., Granada A., Decressin T., Hirschi R., Frischknecht U., Charbonnel C., Maeder A., Grids of stellar models with rotation. I. Models from 0.8 to $120 \mathrm{M}_{\text {sun; }}$ at solar metallicity $(\mathrm{Z}=0.014)$, A\&A, 2012, vol. 537, p. A146

Fox G. K., Stellar occultation of polarized light from circumstellar electrons. III - General axisymmetric envelopes, ApJ, 1991, vol. 379, p. 663

Fox G. K., The theoretical polarization of pure scattering axisymmetric circumstellar envelopes, ApJ, 1994, vol. 435, p. 372 
Gaunt J. A., Continuous Absorption, Royal Society of London Philosophical Transactions Series A, 1930, vol. 229, p. 163

Groh J. H., Meynet G., Ekström S., Massive star evolution: luminous blue variables as unexpected supernova progenitors, A\&A, 2013, vol. 550, p. L7

Harmanec P., Review of observational facts about Be stars, Hvar Observatory Bulletin, 1983 , vol. 7, p. 55

Harmanec P., Stellar masses and radii based on modern binary data, Bulletin of the Astronomical Institutes of Czechoslovakia, 1988, vol. 39, p. 329

Haubois X., Carciofi A., Mota B., Dynamical Evolution of Viscous Disks Around Be Stars. II : Polarimetry, ApJS, 2013

Haubois X., Carciofi A. C., Rivinius T., Okazaki A. T., Bjorkman J. E., Dynamical Evolution of Viscous Disks around Be Stars. I. Photometry, ApJ, 2012, vol. 756, p. 156

Hummel W., Hanuschik R. W., Line formation in Be star envelopes. II. Disk oscillations., A\&A, 1997, vol. 320, p. 852

Isaacs A., Daintith J., Martin E., A dictionary of science. Oxford paperback reference, Oxford University Press, 2003

Karzas W. J., Latter R., Electron Radiative Transitions in a Coulomb Field., ApJS, 1961, vol. 6 , p. 167

Kato S., Low-frequency, one-armed oscillations of Keplerian gaseous disks, PASJ, 1983, vol. 35 , p. 249

Krumholz M. R., Theoretical Models of Massive Star Formation: Predictions for the Circumstellar Environment of Massive Stars. In Circumstellar Dynamics at High Resolution , vol. 464 of Astronomical Society of the Pacific Conference Series, 2012, p. 339

Lee R. W., ed., 1991 A review of spectral line broadening relevant to hot dense plasmas

Lopez-Puertas M., Taylor F. W., Non-LTE radiative transfer in the atmosphere, 2001 
Lynden-Bell D., Pringle J. E., The evolution of viscous discs and the origin of the nebular variables., MNRAS, 1974, vol. 168, p. 603

Meynet G., Maeder A., Stellar evolution with rotation. V. Changes in all the outputs of massive star models, A\&A, 2000, vol. 361, p. 101

Monnier J. D., Zhao M., Pedretti E., Thureau N., Ireland M., Muirhead P., Berger J.-P., Millan-Gabet R., Van Belle G., ten Brummelaar T., McAlister H., Ridgway S., Turner N., Sturmann L., Sturmann J., Berger D., Imaging the Surface of Altair, Science, 2007, vol. 317 , p. 342

Okazaki A. T., Long-term V/R variations of Be stars due to global one-armed oscillations of equatorial disks, PASJ, 1991, vol. 43, p. 75

Okazaki A. T., On the confinement of one-armed oscillations in discs of Be stars., A\&A, 1997, vol. 318, p. 548

Okazaki A. T., Viscous Transonic Decretion in Disks of Be Stars, PASJ, 2001, vol. 53, p. 119

Okazaki A. T., Theory vs. Observation of Circumstellar Disks and Their Formation. In Active OB-Stars: Laboratories for Stellare and Circumstellar Physics, vol. 361 of Astronomical Society of the Pacific Conference Series, 2007, p. 230

Okazaki A. T., Bate M. R., Ogilvie G. I., Pringle J. E., Evolution of truncated decretion disks in Be/X-Ray binaries. In The Physics of Cataclysmic Variables and Related Objects , vol. 261 of Astronomical Society of the Pacific Conference Series, 2002, p. 519

Oudmaijer R. D., Wheelwright H. E., Carciofi A. C., Bjorkman J. E., Bjorkman K. S., Spectrally and spatially resolved $\mathrm{H} \alpha$ emission from Be stars: their disks rotate Keplerian. In IAU Symposium , vol. 272 of IAU Symposium, 2011, p. 418

Padmanabhan T., Theoretical Astrophysics - Volume 1, Astrophysical Processes, 2000

Porter J. M., On outflowing viscous disc models for Be stars, A\&A, 1999, vol. 348, p. 512

Porter J. M., Rivinius T., Classical Be Stars, PASP, 2003, vol. 115, p. 1153 
Pringle J. E., Accretion discs in astrophysics, ARA\&A, 1981, vol. 19, p. 137

Quirrenbach A., Bjorkman K. S., Bjorkman J. E., Hummel C. A., Buscher D. F., Armstrong J. T., Mozurkewich D., Elias II N. M., Babler B. L., Constraints on the Geometry of Circumstellar Envelopes: Optical Interferometric and Spectropolarimetric Observations of Seven Be Stars, ApJ, 1997, vol. 479, p. 477

Rivinius T., Carciofi A., Classical Be Stars, A\&A, 2013

Rivinius T., Štefl S., Baade D., Bright Be-shell stars, A\&A, 2006, vol. 459, p. 137

Rybicki G. B., Lightman A. P., Radiative processes in astrophysics, 1979

Schaller G., Schaerer D., Meynet G., Maeder A., New grids of stellar models from 0.8 to 120 solar masses at $Z=0.020$ and $Z=0.001, A \& A S, 1992$, vol. 96, p. 269

Secchi A., Schreiben des Herrn Prof. Secchi, Directors der Sternwarte des Collegio Romano, an den Herausgeber, Astronomische Nachrichten, 1866, vol. 68, p. 63

Shakura N. I., Sunyaev R. A., Black holes in binary systems. Observational appearance., A\&A, 1973, vol. 24, p. 337

Shu F., The Physics of Astrophysics: Radiation. Series of books in astronomy, University Science Books, 1991

Slettebak A., The Be stars, PASP, 1988, vol. 100, p. 770

Stokes G. G., On the Change of Refrangibility of Light, Royal Society of London Philosophical Transactions Series I, 1852, vol. 142, p. 463

Struve O., On the Origin of Bright Lines in Spectra of Stars of Class B, ApJ, 1931, vol. 73, p. 94

Townsend R. H. D., Owocki S. P., Howarth I. D., Be-star rotation: how close to critical?, MNRAS, 2004, vol. 350, p. 189

Underhill A., Doazan V., B Stars with and without emission lines, parts 1 and 2, 1982 
Štefl S., Carciofi A. C., Baade D., Rivinius T., Otero S., Le Bouquin J.-B., Fabregat J., Okazaki A. T., Rantakyrö F., The 2008+ outburst of the Be star 28 CMa - a multiinstrument study†.InI AU Symposium, vol. 272of I AU Symposium, 2011, p. 430

Vinicius M. M. F., Zorec J., Leister N. V., Levenhagen R. S., $\alpha$ Eridani: rotational distortion, stellar and circumstellar activity, A\&A, 2006, vol. 446, p. 643

von Zeipel H., The radiative equilibrium of a rotating system of gaseous masses, MNRAS, 1924, vol. 84 , p. 665

Waters L. B. F. M., Waelkens C., Herbig Ae/Be Stars, ARA\&A, 1998, vol. 36, p. 233

Williams J. P., Cieza L. A., Protoplanetary Disks and Their Evolution, ARA\&A, 2011, vol. 49 , p. 67

Wisniewski J. P., Draper Z. H., Bjorkman K. S., Meade M. R., Bjorkman J. E., Kowalski A. F., Disk-Loss and Disk-Renewal Phases in Classical Be Stars. I. Analysis of LongTerm Spectropolarimetric Data, ApJ, 2010, vol. 709, p. 1306

Wood K., Bjorkman J. E., Whitney B., Code A., The Effect of Multiple Scattering on the Polarization from Axisymmetric Circumstellar Envelopes. II. Thomson Scattering in the Presence of Absorptive Opacity Sources, ApJ, 1996a, vol. 461, p. 847

Wood K., Bjorkman J. E., Whitney B. A., Code A. D., The Effect of Multiple Scattering on the Polarization from Axisymmetric Circumstellar Envelopes. I. Pure Thomson Scattering Envelopes, ApJ, 1996b, vol. 461, p. 828

Wood K., Bjorkman K. S., Bjorkman J. E., Deriving the Geometry of Be Star Circumstellar Envelopes from Continuum Spectropolarimetry. I. The Case of zeta Tauri, ApJ, 1997, vol. 477 , p. 926

Yudin R. V., Statistical analysis of intrinsic polarization, IR excess and projected rotational velocity distributions of classical Be stars, A\&A, 2001, vol. 368, p. 912 
Apêndices 

Apêndice A

\section{Teoria de Discos Circunstelares}

\section{A.1 Discos Viscosos Alimentados por Taxas Constantes de Decréscimo}

A física da viscosidade é um tópico muito interessante na astrofísica, dado que existem vários sistemas, não só discos de estrelas Be, como mencionados, que são governados pela viscosidade. A descrição da vistosidade é realizada, em geral, pela parametrização de Shakura e Sunyaev (1973). Como não sabiam qual mecanismo poderia gerar uma viscosidade turbulenta para permitir o transporte de momento angular no disco, postularam que ela seria uma fração desconhecida, $\alpha$. A parametrização da viscosidade turbulenta, $\nu$, é fornecida pela expressão,

$$
\nu=\alpha c_{\mathrm{s}} H, \quad 0<\alpha<1 .
$$

Desta maneira, $\alpha$ descreve a relação do tamanho das células de turbulência com a velocidade do som no meio. Com este valor para a viscosidade, a escala de tempo da difusão viscosa torna-se (Carciofi, 2011),

$$
\tau_{\text {dif }}=\frac{v_{\text {crit }}}{\alpha c_{\mathrm{s}}^{2}} \sqrt{r R_{\star}} \approx 20 \text { ano }\left(\frac{0.01}{\alpha}\right) \sqrt{\frac{\mathrm{r}}{\mathrm{R}_{\star}}} .
$$

Estudos da formação e dissipação dos discos ao redor de estrelas Be encontram escalas de tempo típicas de meses a poucos anos (Wisniewski et al., 2010); portanto, um $\alpha$ de ordem 0.1 ou maior é necessário para reproduzir as escalas de tempo observadas.

Há muitas soluções disponíveis para discos viscosos alimentados por taxas constantes de taxas de decréscimo, $\dot{M}$, (Carciofi, 2011). Bjorkman (1997) apresenta a solução para um disco kepleriano isotérmico alimentado a uma taxa constante por um longo período. 
Esta solução fornece uma estrutura geométrica de um disco viscoso com uma distribuição de densidade superficial em coordenadas cilíndricas $(r, \phi, z)$ dada por (Bjorkman, 1997, Okazaki, 2001, Bjorkman e Carciofi, 2005),

$$
\Sigma(r)=\frac{\dot{M}}{3 \pi \alpha c_{\mathrm{s}}^{2}}\left(\frac{G M_{\star}}{r^{3}}\right)^{1 / 2}\left[\left(R_{0} / r\right)^{1 / 2}-1\right]
$$

onde $\dot{M}$ é a taxa de decréscimo, $c_{\mathrm{s}}$ é a velocidade do som no meio (ver capítulo 1 ), $G$ é a constante gravitacional universal e $M_{\star}$ é a massa estelar. Nesta equação, $R_{0}$ está relacionado com o tamanho físico do disco; para modelos dependentes do tempo, por exemplo, (Okazaki, 2007), $R_{0}$ está associado com o crescimento do disco ou, equivalentemente, à idade do mesmo. Portanto, para resolvermos o problema, devemos especificar $\dot{M}, \alpha$ e a idade do disco ou seu tamanho. Podemos expressar a densidade volumétrica por,

$$
\rho(r, z)=\frac{\Sigma(r)}{\sqrt{2 \pi} H(r)} \exp \left[-0.5\left(\frac{z}{H(r)}\right)^{2}\right],
$$

onde $H$ é a escala de altura,

$$
H(r)=\frac{c_{\mathrm{s}}}{v_{\phi}} r .
$$

Antes de partir para a próxima seção, devemos destacar dois casos limites que são úteis para a compreensão dos sistemas dinâmicos estudados no capítulo 5:

1. Disco suficientemente velho $R_{0} \gg R_{\star}, \Sigma \propto r^{-2}$,

2. Para um disco isotérmico, $\Sigma \propto r^{-2}$ e $\rho \propto r^{-3.5}$.

\section{A.1.1 Perfil Estacionário da Densidade Volumétrica no Equador do Disco}

Um exercício útil é o cálculo do perfil estacionário da densidade volumétrica no plano médio do disco, $z=0$. Para isto, adotemos uma lei de velocidade kepleriana,

$$
v_{\phi}=v_{\text {crit }}\left(\frac{R_{0}}{r}\right)^{1 / 2} .
$$

Agora, utilizando a relação entre as densidades superficial e volumétrica,

$$
\Sigma(r)=\int_{-\infty}^{\infty} \rho(r, z) d z=\sqrt{2 \pi} H \rho_{0}
$$


e substituindo a equação A.6 na A.5, obtemos

$$
H(r)=\frac{c_{\mathrm{s}}}{v_{\text {crit }}\left(R_{0} / r\right)^{1 / 2}} r=\frac{c_{\mathrm{s}}}{v_{\text {crit }}\left(R_{0}\right)^{1 / 2}} r^{3 / 2},
$$

e tomando $\rho_{0}=\rho_{0}^{\prime}\left(R_{0} / r\right)^{n}$ na equação A.7 juntamente com o resultado A.8, temos

$$
\Sigma(r)=\sqrt{2 \pi} \frac{c_{\mathrm{S}}}{v_{\text {crit }}\left(R_{0}\right)^{1 / 2}} r^{3 / 2} \rho_{0}^{\prime}\left(\frac{R_{0}}{r}\right)^{n} .
$$

Neste caso, o disco apresenta um perfil de densidade na forma de uma lei de potência apresentando um expoente radial $n=3.5$, ou seja,

$$
\rho(r)=\rho_{0}\left(\frac{R_{0}}{r}\right)^{3.5} \Rightarrow n_{\mathrm{e}}=n_{0}\left(\frac{R_{0}}{r}\right)^{3.5},
$$

onde $n_{\mathrm{e}}$ é a densidade numérica de elétrons e $n_{0}$ é a densidade numérica de partículas na base do disco. 


\section{Apêndice B}

\section{A Razão $B J+/ B J-$}

A figura B.2 apresenta um esquema das emissões do disco e estelar. Estas grandezas podem ser relacionadas de forma simplificada tomando-se o fluxo espalhado como uma função da variação da profundidade óptica,

$$
F_{\nu}^{\mathrm{esp}}=f F_{\nu}^{\star} e^{-\tau_{\mathrm{esp}}^{\mathrm{pre}}+\tau_{\mathrm{esp}}^{\mathrm{pos}}}
$$

onde $f$ é um fator geométrico e, $\tau_{\text {esp }}^{\text {pre }}$ e $\tau_{\text {esp }}^{\text {pos }}$, são as profundidades antes e após o salto. Portanto, podemos representar a polarização do disco como,

$$
P=\frac{F_{\nu}^{\mathrm{esp}}}{F_{\nu}^{\star}+F_{\nu}^{\mathrm{em}}} \simeq \frac{F_{\nu}^{\mathrm{esp}}}{F_{\nu}^{\star}} \simeq f e^{-\Delta \tau},
$$

onde $F_{\nu}^{\mathrm{em}}$ e $F_{\nu}^{\text {esp }}$ são o fluxo emitido e espalhado pelo disco, respectivamente, $F_{\nu}^{\star}$ é o fluxo da estrela e $\Delta \tau=\tau_{\text {pos }}-\tau_{\text {pre }}$ é a variação da profundidade óptica na descontinuidade.

Agora, definindo os pontos de polarização mínima e máxima num espectro polarizado no salto de Balmer como $P_{\mathrm{BJ}}^{+}$e $P_{\mathrm{BJ}}^{-}$(Fig. B.1). A razão dos valores da polarização, nestes pontos, fornece,

$$
\frac{P_{\mathrm{BJ}}^{+}}{P_{\mathrm{BJ}}^{-}}=\frac{B J+}{B J-}=\frac{e^{-\tau^{+}}}{e^{-\tau^{-}}} \propto e^{N_{-}-N_{+}} .
$$

aqui $N_{-}=N_{2}+N_{3}+\ldots$ representa as populações antes e $N_{+}=N_{3}+N_{4}+\ldots$ a soma das populações após o salto de Balmer ${ }^{1}$. Isto, faz com que o tamanho dos saltos aumente, o que, consequentemente, reflete no alargamento do loop de polarização para estes subtipos espectrais. Simplificadamente, o tamanho do salto estará relacionado com a população

\footnotetext{
${ }^{1}$ Como a variação da profundidade óptica de cada uma está associada com a população do nível da descontinuidade, $e^{N_{\mathrm{i}}}$, a dependência com o subtipo espectral se torna importante.
} 


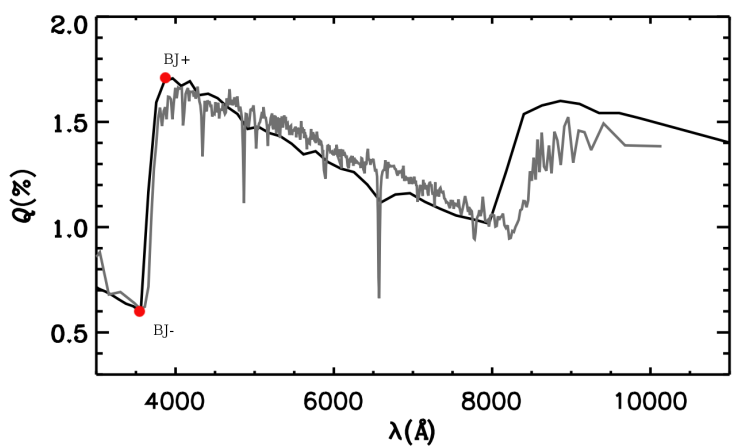

Figura B.1: Espectro polarizado de $\zeta$ Tauri Carciofi et al. (2009).

no nível do salto em cada descontinuidade. Logo, esta mudança das populações exerce grande influência sobre o eixo ordenado do diagrama cor-polarização, conforme variamos o subtipo espectral, o que é apoiado pelos resultados mostrados na seção 3.3.2.

Portanto, devemos conhecer as propriedades da profundidade óptica nos limites da descontinuidade para entender o que a razão fornece.

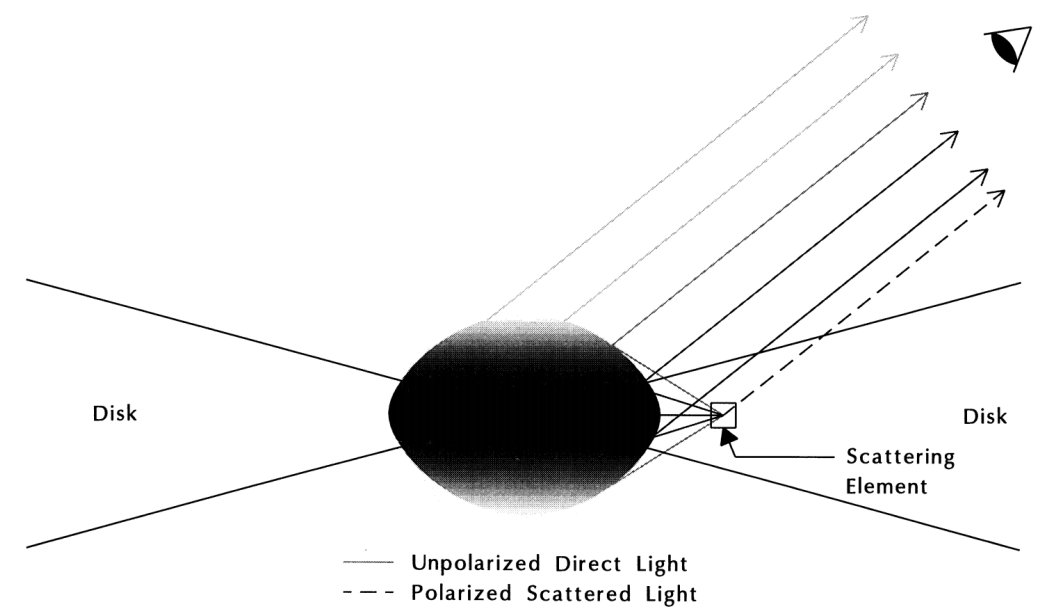

Figura B.2: Diagrama esquemático mostrando a radiação polarizada espalhada por um elemento do disco (linha tracejada) (Bjorkman e Bjorkman, 1994). 
Apêndice C

\section{Absortividades e Opacidades}

Em nosso estudo, adotamos as expressões de Bjorkman e Bjorkman (1994) para a absortividade total do hidrogênio, a qual é representada como a soma da absortividade livre-livre, $\alpha_{\mathrm{ff}}=\rho \kappa_{\mathrm{ff}}$, com a ligado-livre, $\alpha_{\mathrm{bf}}=\rho \kappa_{\mathrm{bf}}$, pela expressão,

$$
\alpha_{\nu}=a_{\mathrm{ff}}\left[1-\exp \left(-\frac{h \nu}{k T}\right)\right] \frac{n_{\mathrm{e}}^{2}}{\nu^{3} T^{1 / 2}}+\left\{\begin{array}{cl}
n(r) N_{3} a_{3}\left(\frac{\nu_{3}}{\nu}\right)^{3} & \nu_{2}>\nu>\nu_{3} \\
n(r) N_{2} a_{2}\left(\frac{\nu_{2}}{\nu}\right)^{3}+n(r) N_{3} a_{3}\left(\frac{\nu_{3}}{\nu}\right)^{3} & \nu>\nu_{2}
\end{array}\right.
$$

onde $N_{2}$ e $N_{3}$ são as populações do segundo e terceiro nível, $a_{2}$ e $a_{3}$ são seus coeficientes e $\nu_{2}$ e $\nu_{3}$ são as frequências dos saltos de Balmer e de Paschen.

\section{C.1 Métodos de Determinação das Opacidades}

No primeiro método, usamos os valores equatoriais de temperatura, das populações até o vigésimo quinto nível do hidrogênio, a densidade, a fração de ionização e a distância à estrela. Por meio destes valores, determinamos as diferentes opacidades individualmente. Para isto, consideramos cada uma das opacidades ligadas em cada modelo, obtendo resultados que representam os efeitos das profundidades ópticas de forma independente uma das outras. Isso nos permitiu garantir uma representação de como elas variam com o comprimento de onda nas regiões das descontinuidades de interesse.

No segundo método, o cálculo da profundidade óptica é realizado com todas as opacidades ligadas. Portanto, esperamos que haja uma influência recíproca significativa entre as diferentes opacidades, quando comparado com o primeiro método. Por exemplo, a profundidade livre-livre sofre uma queda acentuada, tornando-se $\sim 10^{6}$ menor que a obtida pelo primeiro método. 
A vantagem deste segundo método é que podemos obter os parâmetros necessários para calcular as diferentes profundidades ópticas em diferentes regiões do disco, ao contrário do primeiro, o qual nos fornece uma ideia da ordem de grandeza das profundidades em diferentes comprimentos de onda.

\section{C.2 Dependência Radial das Profundidades Ópticas}

Para determinar a dependência da profundidade óptica em um dado raio e num dado comprimento de onda precisamos das populações dos níveis, da densidade numérica, da temperatura, da fração de ionização naquela região e dos valores dos fatores de Gaunt (apêndice C.3). Além disso, assumimos a lei de densidade radial para um disco com período de perda de massa suficientemente longo (eq. A.10). Com o intuito de simplificar, a dependência radial dos outros parâmetros, como a temperatura e a fração de ionização, foram considerados constantes.

\section{C.2.1 Profundidade Óptica Eletrônica}

Estudamos previamente os resultados das simulações. Desta forma, pudemos estimar a fração de ionização como sendo $f \sim 1$, ou seja, praticamente todo o material circunstelar está ionizado para todos os tipos espectrais estudados. Consideramos, por isto, apenas a dependência radial da densidade no cálculo dos perfis das absortividades. Para calcular a profundidade óptica eletrônica aplicamos a fórmula de espalhamento de Thomson,

$$
\tau_{\mathrm{e}}=\sigma_{\mathrm{T}} \int_{1 R_{\star}}^{r} f n_{\mathrm{e}}\left(r^{\prime}\right) d r^{\prime}
$$

onde $f$ é a fração de ionização, $n_{\mathrm{e}}$ é a densidade numérica eletrônica e $\sigma_{\mathrm{T}}$ é a seção de choque de Thomson,

$$
\sigma_{\mathrm{T}}=\frac{8 \pi}{3} r_{0}^{2}\left[\mathrm{~cm}^{2}\right]
$$

Utilizando a relação A.10 na equação C.2, obtemos a profundidade óptica eletrônica,

$$
\tau_{\mathrm{e}}=\sigma_{\mathrm{T}} \int_{1 R_{\star}}^{r} f n_{0}\left(\frac{R_{\star}}{r^{\prime}}\right)^{3.5} d r^{\prime}=\frac{2}{5} \sigma_{\mathrm{T}} f n_{0} R_{\star}\left[1-\left(\frac{R_{\star}}{r}\right)^{2.5}\right]
$$




\section{C.2.2 Profundidade Óptica Livre-Livre}

Adotamos o primeiro termo da expressão C.1 utilizando o segundo método. Deste método, o valor máximo é atingido quando $\tau \sim 10^{-7}$. A causa deste baixo valor está na presença de outras fontes de opacidade. A contribuição obtida pelo primeiro método para este comprimento de onda foi $\sim 0.1$. Deste método o valor máximo é atingido em $\tau \sim 10^{-7}$, a causa do baixo valor está na presença de outras opacidades ${ }^{1}$. Para obter a dependência radial da absorção livre-livre, fazemos

$$
\tau_{\mathrm{ff}}=\int_{1 R_{\star}}^{r} \alpha_{\mathrm{ff}} d r^{\prime}=\int_{1 R_{\star}}^{r} a_{\mathrm{ff}} \frac{Z^{2} f n^{2}(r)}{\nu^{3} T^{1 / 2}}\left[1-\exp \left(\frac{h \nu}{k T}\right)\right] d r^{\prime} .
$$

Primeiro, considerando $h \nu<<k T$ e fazendo $\tau_{\text {ff }}=\tau_{\text {ff }}(n)$, obtemos a relação aproximada

$$
\tau_{\mathrm{ff}}=\int_{1 R_{\star}}^{r} a_{\mathrm{ff}} \frac{Z^{2} f n^{2}(r)}{\nu^{3} T^{1 / 2}} \frac{h \nu}{k T} d r^{\prime}=a_{\mathrm{ff}} \frac{Z^{2} f h R_{\star} n_{0}^{2}}{\nu^{2} T^{3 / 2} k} \int_{1 R_{\star}}^{r} n^{2} d r^{\prime},
$$

após a integração,

$$
\tau_{\mathrm{ff}}=a_{\mathrm{ff}} \frac{Z^{2} f h R_{\star} n_{0}^{2}}{\nu^{2} T^{3 / 2} k}\left[1-\left(\frac{R_{\star}}{r}\right)^{6}\right] .
$$

\section{C.2.3 Profundidade Óptica Ligado-Livre}

Observamos, de simulações preliminares, que a população dos níveis não varia muito com o raio. Por isso, assumimos uma independência radial da população do segundo e terceiro níveis do hidrogênio, $N_{2}$ e $N_{3}$, e outros parâmetros neste cálculo. Para calcular a dependência radial da absortividade ligado-livre partimos do segundo termo da equação C.1. Inserindo a equação A.10 na equação C.1 a profundidade óptica torna-se,

$$
\tau_{\mathrm{bf}}=\left\{\begin{array}{cl}
n_{0} R_{\star} N_{3} a_{3}\left(\frac{\nu_{3}}{\nu}\right)^{3}\left[1-\left(\frac{R_{\star}}{r}\right)^{2.5}\right] & \nu_{2}>\nu>\nu_{3} \\
n_{0} R_{\star}\left[N_{2} a_{2}\left(\frac{\nu_{2}}{\nu}\right)^{3}+N_{3} a_{3}\left(\frac{\nu_{3}}{\nu}\right)^{3}\right]\left[1-\left(\frac{R_{\star}}{r}\right)^{2.5}\right] & \nu>\nu_{2}
\end{array}\right.
$$

\section{C.3 Determinação dos fatores de Gaunt}

Antes de calcularmos os profundidades ópticas precisamos determinar os fatores de Gaunt para o cálculo das absortividades livre-livre e ligado-livre. Karzas e Latter (1961)

\footnotetext{
${ }^{1}$ Pelo primeiro método o máximo obtido foi de 0.1 .
} 
mostraram (Fig. C.1(a)) que para elétrons com energia incidente, $\log \left[E_{\mathrm{i}} /\left(Z^{2} R_{\mathrm{y}}\right)\right]$, que em nosso caso é $\sim 1$ o $g_{\mathrm{ff}}$ é aproximadamente 1. Outra opção é por meio da figura C.1(b) onde, pelo cálculo da energia dos fótons, $\log \left[(h \nu) /\left(Z^{2} R_{\mathrm{y}}\right)\right]$ obtemos o mesmo valor. Aqui $R_{\mathrm{y}}$ é o número de Rydberg.

Eles fizeram o mesmo cálculo para as transições ligado-livre. Para $n=3$ os seguintes subníveis são apresentados: $3 s, 3 p$ e $3 d$, para o primeiro temos $g_{\mathrm{bf}} \sim 6$ (veja Fig.7 (Karzas e Latter, 1961)), para o segundo $~ 1$ (ver Fig.9 (Karzas e Latter, 1961)) e para o último $\sim 7$ (ver Fig.11 (Karzas e Latter, 1961)). Com estes valores, podemos calcular o fator de Gaunt médio, $\bar{g}_{\text {bf }}$, este valor é mostrado em Gaunt (1930), para o nível 3 o valor é 0.92 . As figuras C.1(c) e C.1(d) fornecem para o fator de Gaunt médio ligado-livre o valor $\sim 1$. 


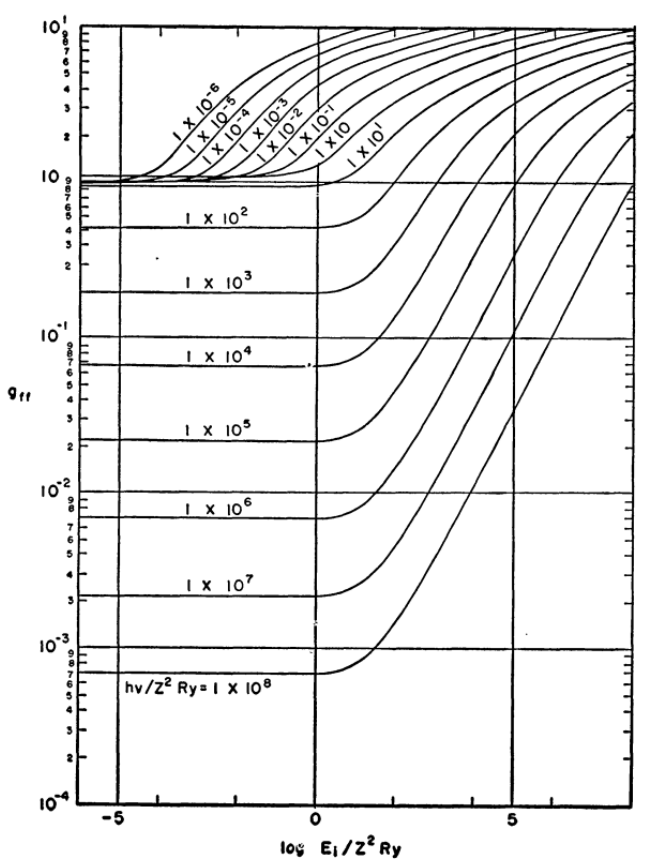

(a)

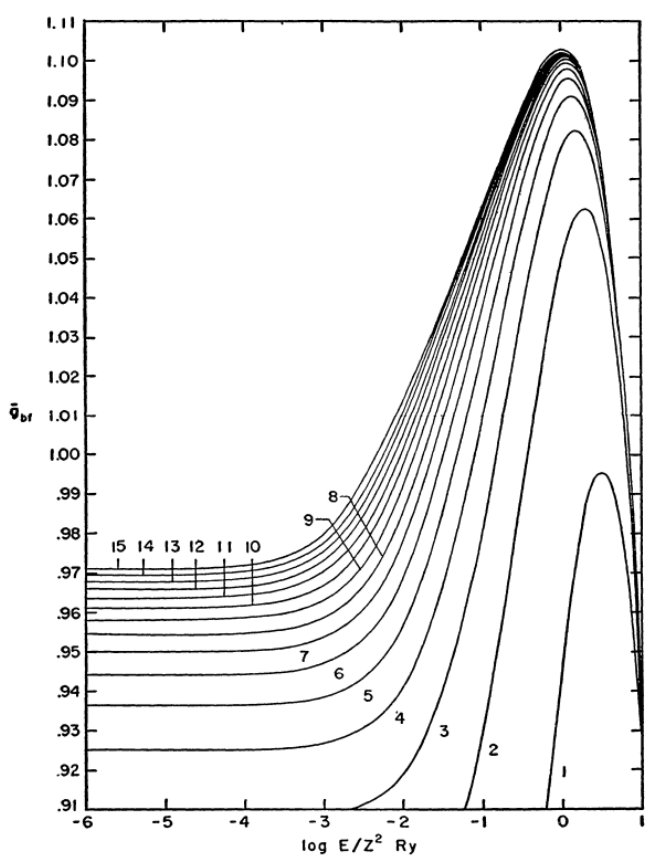

(c)

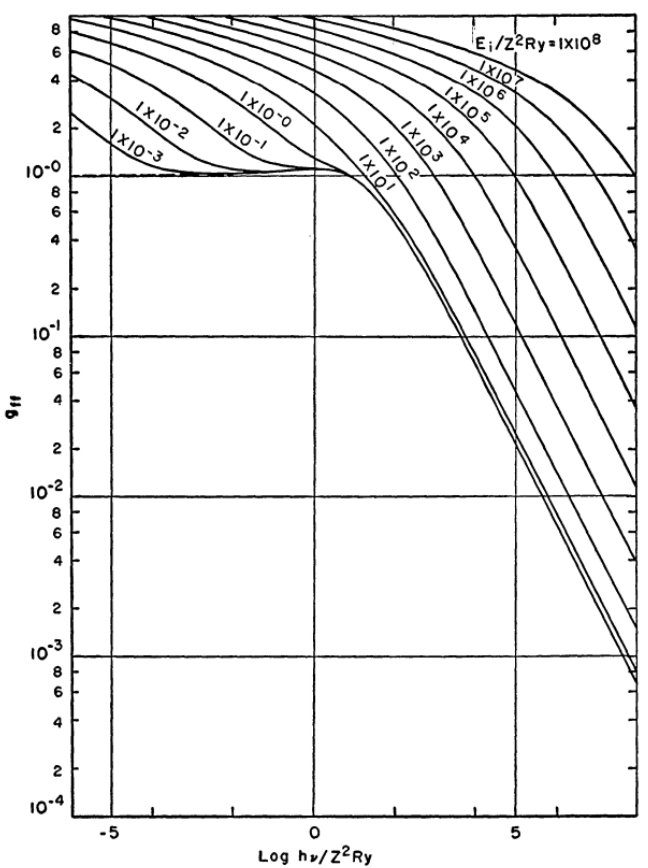

(b)

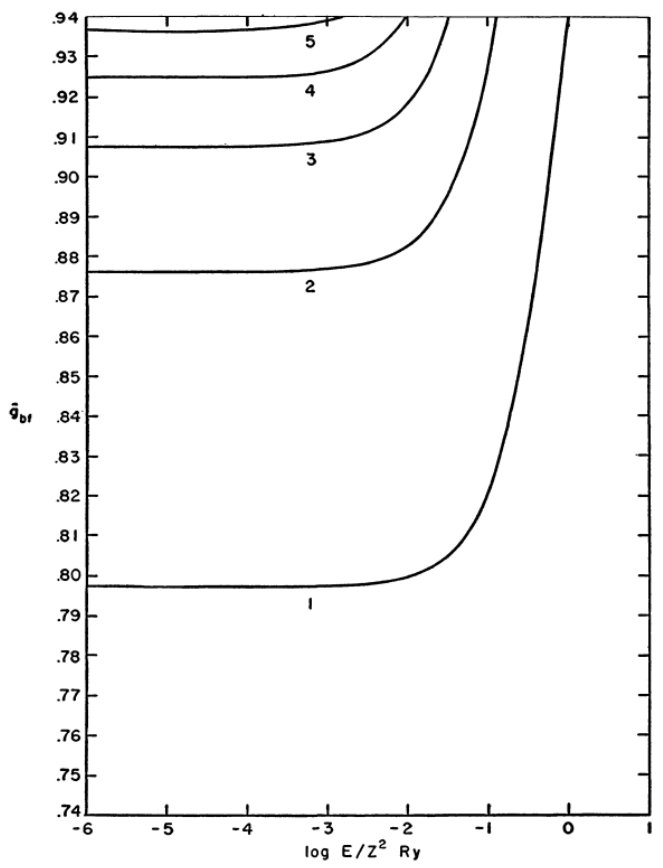

(d)

Figura C.1: Superior: (a) O fator de Gaunt livre-livre versus a energia incidente dos elétrons para vários valores de energia de fótons, (b) o fator de Gaunt livre-livre versus a energia dos fótons para vários energias de elétrons incidentes, Karzas e Latter (1961). Inferior: (c) Fator de Gaunt médio ligado-livre sobre uma camada versus a energia de um elétron livre para $n=1 \rightarrow 15$, (d) fator de Gaunt médio ligado-livre versus a energia do fóton para várias energias de elétrons incidentes, (Karzas e Latter, 1961). 
Apêndice D

\section{O Salto de Balmer}

O salto de Balmer está associado à fotoionização de um elétron da órbita de nível $n=2$ de um átomo de hidrogênio. Neste orbital, o elétron possui energia $E_{2}=-\left(13.6 / 2^{2}\right) \mathrm{eV}=$ $-3.40 \mathrm{eV}$, ou seja, um fóton deve possuir uma energia igual ou superior a $3.40 \mathrm{eV}$ para fotoionizar este átomo. Portanto, qualquer fóton cujo comprimento de onda seja $\lambda \leq$ $(h c / 3.40) \mathrm{eV}=3646 \AA$ pode fotoionizar um átomo de hidrogênio no primeiro estado excitado, $n=2$. Assim, caso uma fonte apresente um espectro "deslocado" para esta região, a opacidade do material estelar repentinamente aumentará para comprimentos de onda $\lambda \leq 3646 \AA$ e, consequentemente, o fluxo radiativo medido para a estrela decrescerá abruptamente. A queda no espectro contínuo da estrela, neste comprimento de onda, recebe o nome de salto de Balmer (Fig. 1.3).

O tamanho do salto de Balmer em estrelas quentes depende da fração de átomos de hidrogênio que estão no primeiro estado excitado, $n=2$. Desta forma, o salto será mais acentuado quando a fração da população do segundo nível pela total, $N_{2} / N_{\text {total }}$, for máxima. Esta quantidade pode ser determinada pela equação de Boltzmann, da qual podemos obter a temperatura,

$$
\frac{N_{\mathrm{b}}}{N_{\mathrm{a}}}=\frac{g_{\mathrm{b}} e^{-E_{\mathrm{b}} / k T}}{g_{\mathrm{a}} e^{-E_{\mathrm{a}} / k T}}=\frac{g_{\mathrm{b}}}{g_{\mathrm{a}}} e^{-\left(E_{\mathrm{b}}-E_{\mathrm{a}}\right) / k T},
$$

onde $k$ é a constante de Boltzmann, $g$ são os pesos estatísticos de cada nível, $T$ é a temperatura e $E$ a energia do nível. 
Apêndice E

\section{O Efeito do Espalhamento Múltiplo na Polarização de Envelopes Circunstelares}

Wood et al. (1997) apresentam um estudo do espalhamento múltiplo em envelopes circunstelares axissimétricos $^{1}$ de diferentes geometrias. Este trabalho mostra que o aumento da polarização ocorre por causa de múltiplos espalhamentos predominantemente dentro de um disco opticamente fino. Por causa da geometria do disco, estes espalhamentos múltiplos reduzem a componente do vetor campo elétrico paralelo ao seu plano, deixando um aumento líquido na polarização que é perpendicular ao disco, ou seja, espalhamentos múltiplos são responsáveis pelo aumento dos níveis de polarização em discos circunstelares.

Na figura E.1, apresentamos um resultado deste estudo. A figura mostra a variação da profundidade óptica equatorial, $\tau_{\text {eq }}$, em relação à polarização, $Q(\%)$, proveniente do espalhamento simples para um envelope com um ângulo de abertura de $45^{\circ}$. Nota-se que $Q(\%)$ cresce até um máximo e depois diminui atingindo valores negativos. Isto ocorre, porque $\tau_{\text {eq }}$ elevados diminuem a quantidade de fótons que escapam do disco. Consequentemente, ocorre uma alta atenuação do fluxo equatorial, fazendo a maior parte dos fótons observados passarem a ser das regiões polares menos densas. Estes fótons estão polarizados opostamente aos fótons que são espalhados no disco, resultando num efeito de cancelamento. Então, conforme aumentamos $\tau_{\text {eq }}$, a polarização começa a diminuir, tornando-se negativa quanto os elétrons espalhados nos polos passam a dominar.

\footnotetext{
${ }^{1}$ Com simetria em relação a um eixo.
} 


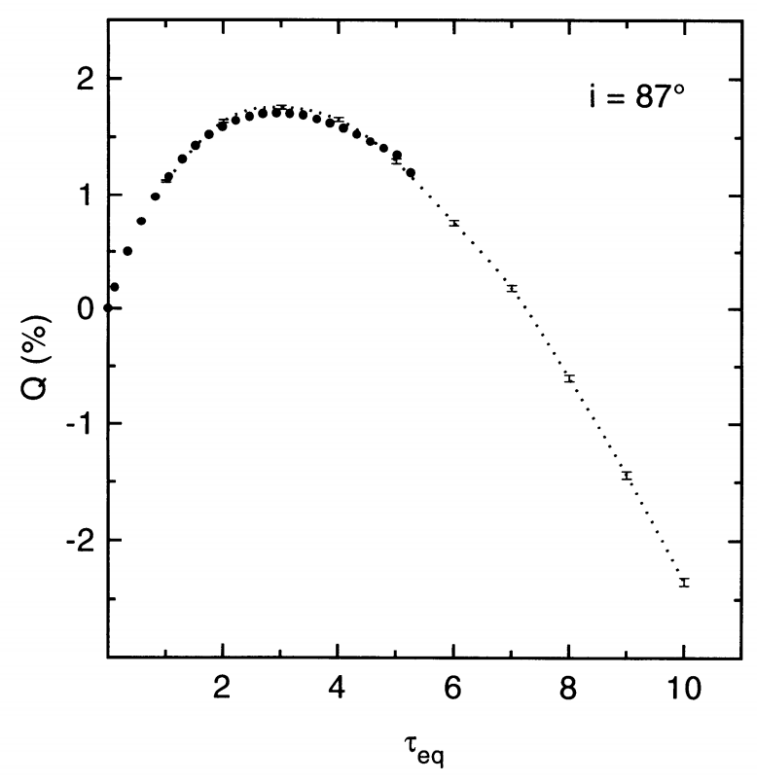

Figura E.1: Polarização como função da profundidade óptica equatorial para várias inclinações, para um disco com ângulo de abertura de $45^{\circ}$ (Wood et al., 1996b), os círculos maiores são os resultados do espalhamento simples mais atenuação investigados por Fox (1994). 
Apêndice $F$

Artigo 



\title{
DYNAMICAL EVOLUTION OF VISCOUS DISKS AROUND BE STARS. II : POLARIMETRY.
}

\author{
X. Haubois, A.C. Carciofi, B. Mota. \\ Instituto de Astronomia, Geofísica e Ciências Atmosféricas, Universidade de São Paulo, Rua do Matão 1226, Cidade Universitária, São Paulo, SP 05508-900, \\ Brazil \\ AND \\ Z.H. DraPER AND J.P. WiSNIEWSKI \\ Department of Astronomy, University of Washington, Box 351580 Seattle, WA 98195, USA
}

\begin{abstract}
Be stars exhibit variability for a great number of observables. Putting the pieces together of the dynamics at play is not an easy task and requires arduous modeling before achieving a good fit of the observational data. In order to guide the modeling process and make it more efficient, it is very instructive to investigate reference dynamical cases. This paper is the second of a series that aims to demonstrate the capacity of deriving the dynamical history and fundamental parameters of a classical Be star through the follow-up of various observable quantities. After a short review on the origin of polarized signals from Be stars, we investigate predictions for polarimetric observables in the continuum at different bands. Our models are based on a coupling of a dynamic viscous decretion simulation in a disk and a 3-D non-LTE radiative transfer. Introducing the new concept of polarization color loop (PCL), we show that certain combinations of polarimetric observables exhibit features that are characteristic of a mass loss history. They also enable to estimate fundamental parameters such as the inclination angle and the $\alpha$ viscous diffusion parameter. We therefore present the PCL as a powerful diagnosis tool to understand the dynamics in a Be star disk and test it with observations.
\end{abstract}

Subject headings: circumstellar matter radiative transfer stars: emission-line, Be

\section{INTRODUCTION}

Be stars are non-supergiant, early-type stars with a circumstellar (CS) disk that is created from matter ejected from the star. Recent observational facts brought by spectrointerferometry and spectro-astrometry (e.g. ??) supports the fact that so far all studied Be star disks rotate in a Keplerian fashion. This characteristic, together with other observational signatures of the disk outlined in ?, are properties that only the viscous decretion disk (VDD) model can reproduce. This model, first suggested by ? and further developed by ?, ?, ?, ? and ?, among others, uses the angular momentum transport by turbulent viscosity to lift material into higher orbits, thereby causing the disk to grow in size. This model has already been successfully applied to systems showing stable continuum emission: e.g. $\zeta$ Tauri (?), $\chi$ Oph (?) and $\beta$ CMi (?) and systems exhibiting a more variable photometric activity (28 CMa, ?).

Polarization is a powerful tool to study the geometry of the disk (opening angle, flaring) of Be stars without angularly resolving it. Polarized flux originates from electron scattering off the disk, and is affected by both pre- and post-scattering absorption by H I atoms (??). Since H I opacity depends on the physical state of the gas, studying polarimetric observables at different wavelengths allows one to probe different regions of the disk. In the literature, the polarimetric technique has an established history of providing a unique diagnosis in identifying and studying the detailed CS environments of Be stars (????).

Haubois et al. 2012 (hereafter Paper I) studied the temporal variability of Be disks, based on SINGLEBE VDD hydrodynamics simulations (?). SINGLEBE solves the 1-D surface density evolution equation for a viscous decretion disk. The effects of variable mass injection rates on the disk structure, and their corresponding effect on the photometry, were stud-

Electronic address: xhaubois@astro.iag.usp.br ied at different wavelengths and compared to observations. More specifically, we first studied the different timescales that characterize the evolution of the disk surface density and how this surface density responds to changes in the mass injection rates. These surface density profiles were used as inputs to the three-dimensional non-LTE Monte Carlo radiative transfer code HDUst (??) that allowed the calculation of photometric observables at various wavelengths. The characteristic shapes of these lightcurves agree qualitatively well with observations, which provide strong circumstantial evidence that viscosity is indeed the mechanism that redistributes matter along the CS disk. A first successful confrontation of theoretical VDD lightcurves with observations was done by ? for the Be star $28 \mathrm{CMa}$. To summarize, Paper I provides the reader with a description of the photometric variability from a Be star in the framework of the VDD model. With the present paper, we aim at exploring the variability of the continuum polarimetric features in the same manner.

In $\S ? ?$, we describe the polarigenic mechanisms operating in Be disks. Then we present the dynamical models we investigated and their signatures on common polarimetric observables. We also show the diagnosis potential of a series of diagrams that we named polarization color loops (PCLs) that represent a powerful tool to follow the mass injection history in Be stars (§??). Finally, a discussion and a comparison to observed data of these synthetic observables are presented in $\S ?$ ? before concluding.

\section{POLARIZATION IN BE STAR DISKS}

Spectropolarimetric observations of Be stars usually reveal a sawtooth pattern that displays abrupt changes of the polarization close to the H I ionization thresholds (see, e.g., ?, for examples of observed polarization spectra). It is useful to review the origin of this pattern to understand precisely the physical processes that control the shape of the polarized spectrum. In this section, we therefore adopt an analytical 
model to describe a viscous decretion disk surrounding a rotationally deformed and gravity darkened B2 star (see Table 1 of Paper I for a description of the adopted stellar parameters). For the disk, we adopted the VDD model in its simplest form: after a sufficiently long and stable period of mass decretion, a viscous disk assumes a power-law density profile given by $\rho=\rho_{0}\left(R_{\star} / r\right)^{3.5}(?)$, where $\rho_{0}$ is the density at the base of the disk. In this study and in the rest of the paper, we keep the disk outer radius fixed at $20 R_{\star}$.

Figure 1 shows two theoretical polarized spectra, at two different base densities and for an inclination angle of $70 \mathrm{deg}$. While the low density model has a nearly flat spectrum in the optical and near IR, the high density model displays a steep spectrum with marked changes in the $\mathrm{H}$ I ionization thresholds. This behavior can be understood in terms of the relative contribution to the total opacity of each opacity source. Figure 1 shows the total optical depth of the disk, measured in the radial direction along the midplane (orange lines). The contribution of the absorptive (free-free and bound-free) and scattering (Thomson) opacities are also shown.
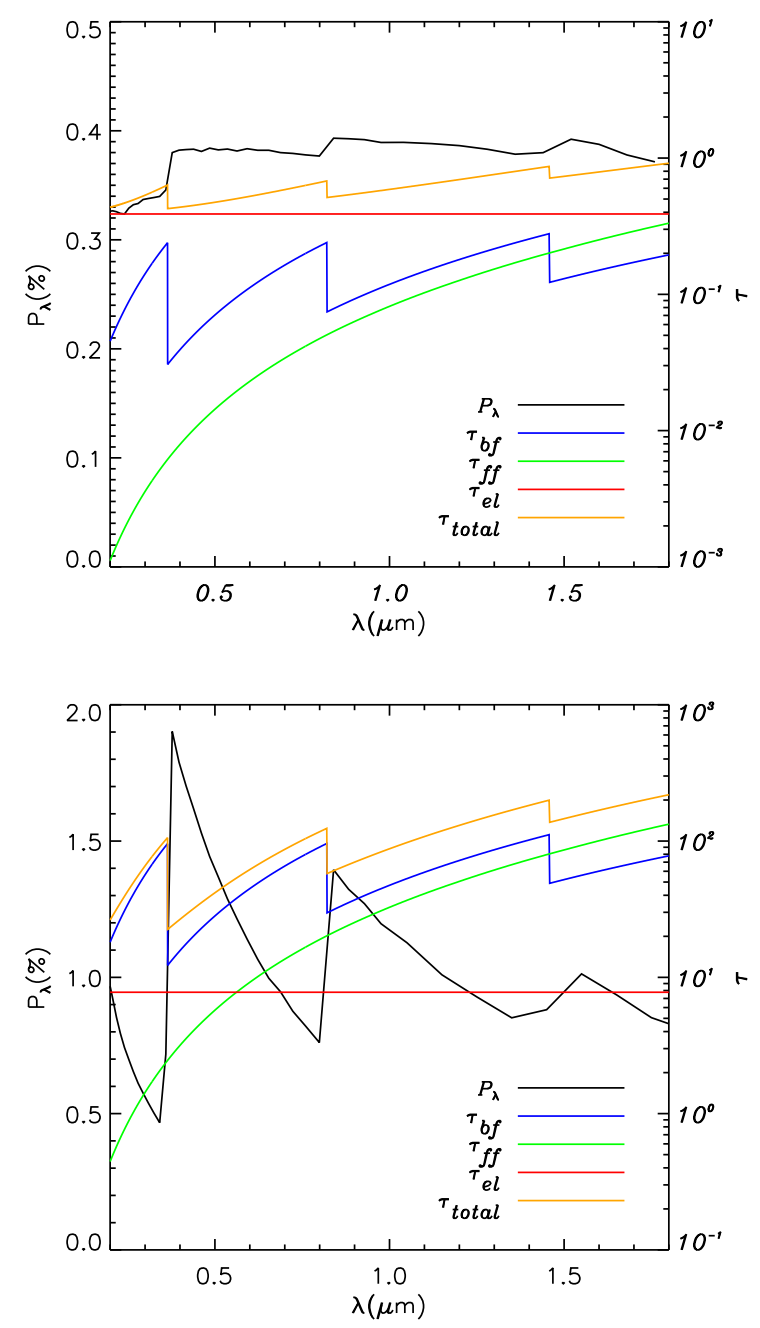

FIG. 1.- Polarized spectrum and radial optical depth contributions along the midplane. The total optical depth is the sum of the optical depth for each continuum opacity source, as indicated. Upper panel: $1.5 \times 10^{-11} \mathrm{~g} \mathrm{~cm}^{-3}$; lower panel: $3 \times 10^{-10} \mathrm{~g} \mathrm{~cm}^{-3}$. The inclination angle is 70 degrees.
At low density, the electron scattering opacity, which is wavelength independent, is responsible for most of the total opacity. The resulting polarized spectrum is consequently nearly flat. However, changes in the polarization level occurs close to $\mathrm{H}$ I ionization thresholds, more importantly at the Balmer jump $(0.365 \mu \mathrm{m})$. What causes the decrease in the polarization redward of the jumps is the increase in the H I opacity. This effect is thoroughly discussed in ?, and can be understood in terms of pre-scattering (and to a lesser degree, post-scattering) absorption of starlight, that decreases the polarized flux and hence the polarization level, creating an anti-correlated aspect of the optical depth and polarization curves. If the density of the disk increases, the electron opacity will increase more or less linearly, as it is proportional to the number density of free electrons. However, the boundfree and free-free opacities, being roughly proportional to the square of the density (?) gehrz, will increase much faster than the electron opacity. Therefore, at a high disk density (lower panel of Fig. ??), the bound-free opacity dominates the total opacity. As a result, the polarized spectrum, besides displaying much more pronounced changes in the H I jumps, has a quite steep slope that results from the spectral dependency of the bound-free opacity.

Another important aspect to consider is the radial dependence of the opacities, which is shown in Fig. ?? for a wavelength blueward of the Balmer discontinuity and for three spectral types of the central star (B0, B2 and B4).

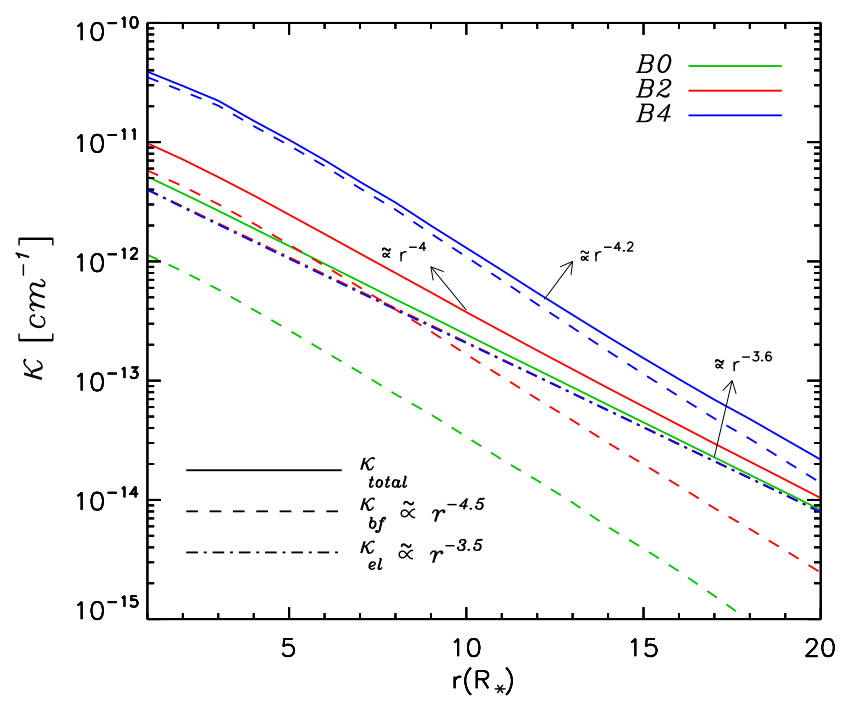

FIG. 2.- Opacities redwards of the Balmer discontinuity as a function of the distance to the star surface. The calculations assume a rotating B2 star surrounded by a viscous decretion disk with a base density of $\rho_{0}=$ $3 \times 10^{-11} \mathrm{~g} \mathrm{~cm}^{-3}$ should be the same as figure 4 . The free-free opacity is not shown because for short wavelengths it is much smaller than the other opacities. The electron scattering opacities are very similar for the three spectral types so that their curves overlap.

Naturally, the slope of the electron opacity curve follows the slope of the density $\left(\propto r^{-3.5}\right)$, because, for the models shown, H I is more than $99.99 \%$ ionized everywhere in the disk (in other words, the ionization fractions are close to $100 \%$, and therefore the electron number density is proportional to the total density). However, the bound-free opacity falls much steeper than the electron opacity. For the B2 model, $\tau_{\mathrm{bf}} \propto r^{-4.5}$, and no significant difference in slope was 
found for different spectral types. What sets this slope is the radial dependence of the non-LTE H I level populations (see Carciofi \& Bjorkman 2006, for a discussion). Even though the slope of the bound-free opacity is similar for the three spectral types shown, the actual levels are rather different: the bound-free opacity increases fast as one goes from early to late type as a result of the decrease in the ionization fraction. This is explained by the deficit of UV ionizing photons for later spectral types. As a result, for a B0 star, electron scattering dominates the opacity at $0.3647 \mu \mathrm{m}$ everywhere in the disk, and the opposite is true for the B4 star. An intermediate behavior is seen for the B2 star model: while the bound-free opacity dominates in the inner disk (up to $\approx 7 R_{\star}$ ), the opacity is controlled by electron scattering in the outer disk.

The effects of the strong dependence of the relative weight of the different opacities with spectral type can be seen in Fig. ??., that shows the polarized spectra associated with the models shown in Fig. ??. The spectrum goes for a relatively flat one for the B0 star (electron scattering dominated) to a steep one, with marked discontinuity at the H I thresholds, for a B4 star at the density considered.

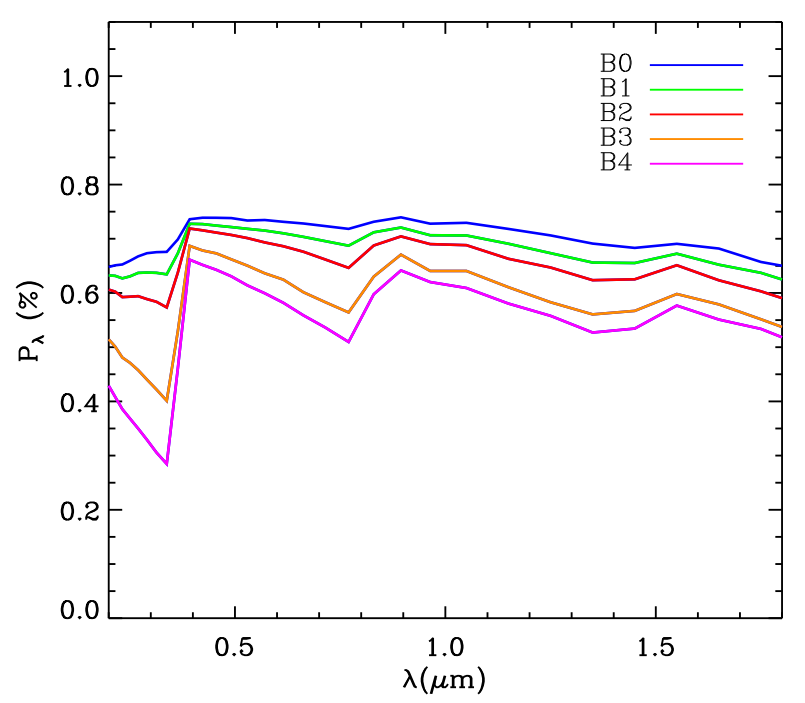

FIG. 3.- Polarized spectra for 5 spectral types. density is $10^{1} 4$ partculas.cm $(-3)$.

Another interesting aspect seen in Fig. ?? is the fact that the polarization level for a given wavelength increases with the effective temperature. This is seen more qualitatively in Fig. 4 that shows how the $V$-band maximum polarization of a given model (that usually happens for $i \approx 70 \mathrm{deg}$ ) varies with spectral type.

Moreover, the temperature distribution along the disk affects the density distribution. As a result of these coupled effects on the total opacity, the polarized levels from the disk will vary depending on the spectral type of the central star. Figure ?? shows this dependence for different densities at the base of the disk.

For low densities, all models (including the coolest ones) are fully ionized, the scattering mass does not vary with the spectral type and the curve on Figure ?? is flat. However for larger densities the disk is no longer fully ionized and as the bound-free absorption coefficient decreases strongly towards

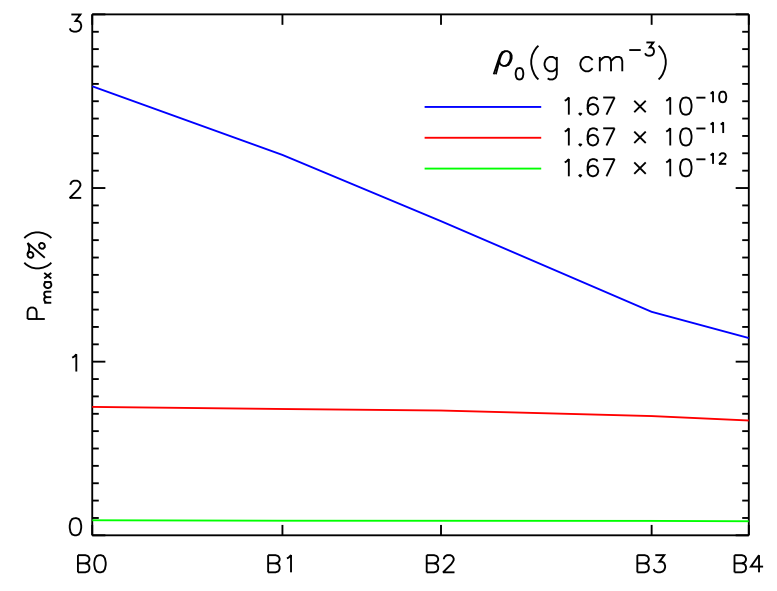

FIG. 4.- $V$-band maximum level of polarization versus the effective temperature of the star for three different densities. The inclination angle is $70^{\circ}$.

early spectral types (Figure ??), the polarization increases accordingly.

In ?, a similar approach was undertaken using the code BEDISK (?). Based on an self-consistent treatment of the thermal structure of the disk, they performed a radiative transfer in a gaseous disk that feeds a Monte Carlo multiple scattering routine that provides polarization levels for different parameters. For a B2V star, the predicted polarization levels for different base densities (Figure 1, ?) are qualitatively similar to the ones we present in this paper. At the difference of the here-presented results, it is noteworthy they find qualitatively similar polarization levels for other spectral types.

We conclude that the polarization spectrum carries valuable information about the circumstellar disk. The polarization level at a given wavelength depends primarily on the total scattering mass of the envelope (number of free electrons), while the shape of the spectrum (its slope or, equivalently, the size of the polarization change across the $\mathrm{H}$ I ionization thresholds) depends on the $\mathrm{H}$ I bound-free opacity, which is a strong function of both the disk density and the spectral type.

\section{DYNAMICAL SIGNATURES IN POLARIMETRY}

In the last section we set the stage for explaining the origin of polarized spectra from Be stars using a VDD model in a steady state (constant mass decretion rate).

However, Be stars are known to be highly variable, and a constant mass decretion rate is likely to be the exception rather than the rule. A much more common situation is a disk whose characteristics are time dependent, in response to a mass decretion rate that changes with time.

To model the signature of a time-dependent VDD, we follow the same approach used in paper I. In that paper, the photometric variability was studied in detail and we showed that predictions based on simple, yet realistic, mass injection rate scenarios agree quite well with the correlations observed for both shell and Be stars (we refer the reader to the discussion in $\S 5$ of Paper I). Here, our goal is to extend that study to the two main polarimetric observables discussed in $\S 2$ :ALEX: that confirms we have to explain the link between opacities and observables in sect 2 the polarization level in $V$ band, and 
the polarization change across the $\mathrm{H}$ I ionization threshold (or, what is equivalent, the slope of the polarized spectrum).

We first started by simulating the variations of the surface density structure in a viscous isothermal Keplerian disk with SINGLEBE. This code computes temporal series of surface density profiles for a given dynamical scenario. This latter is built from the history of the mass injection into the disk and a coefficient of viscosity $(\alpha, ?)$. In Paper I, we investigated in details the behavior of surface density radial profiles for different dynamical scenarios: monotonic building-up and dissipation phases of the disk, periodic and episodic outbursts. We established that the $\alpha$ parameter determines the timescale of the variations of those surface density profiles. Those variations often represent a situation where the size of the accretion and decretion regions oscillates back and forth, in reaction to the variable mass injection into the disk. To transform these structural informations into observables, we used the surface density profiles as input to the radiative transfer code HDUST for each epoch of the dynamical scenario we wanted to investigate. This 3-D Monte Carlo code produces a full spectral synthesis of a star+disk system with a non-LTE treatment and produces output spectra for the Stokes parameters $Q$ and $U$.

To understand the effects of dynamical scenarios upon polarimetric observables we start with a case of uninterrupted disk build-up and dissipation phases.

\subsection{Disk build-up and dissipation}

\subsubsection{Polarization at the Balmer Discontinuity and in V-band}

The disk build-up and dissipation scenarios are simulated using a constant mass injection rate (it is either a constant value for the building-up phase or 0 for the dissipation). To explore the limit behavior of the disk in these two phases, we study a disk fed by a constant mass injection rate for 50 years, followed by 50 years of a quiescent phase, during which the disk dissipates. To explore the two polarimetric features outlined in $\S 2$, we plot in Fig. ?? the temporal evolution of $P_{V}$ and $P_{B D}$. Results are shown for two $\alpha$ parameters and three inclination angles.

Understanding the build-up phase (upper panels of Figure ??) is straightforward: the polarization level monotonically increases as the disk builds up, reaching a limit value after a time that depends on the $\alpha$ coefficient (quasi-steady state value, for more details see Paper I). The maximum of the polarization level isn't reached at 90 degrees but rather at 70 degrees (e.g., ??). Indeed, at high inclinations, photons scattered in directions parallel to the disk plane are much likely to be absorbed by the disk than photons scattered away from the disk.

The dissipation (lower panels of Figure ??) is characterized by an increase of the $P_{V}$ signal right after the mass injection has been set to zero (no more mass injected at the base of the disk). It is explained by the fact that the (unpolarized) emission in the $V$-band decreases soon after the mass injection stops (see Paper I). However, the total polarized flux decreases less rapidly because it is produced in a bigger area in the disk. This causes the polarization fraction to increase. When a sufficient fraction of the disk mass has been dissipated, there is less and less electrons available for Thomsonscattering, the polarization fraction naturally decreases as is the case for $P_{B D}$. These variations are also naturally $\alpha$ and inclination angle dependent. Eventually, all the polarized signals reach the zero value, when the disk has dissipated all or almost all its scattering mass.

\subsubsection{Polarization color loops (PCLs)}

From Figure 12 of Paper I, we can see the excess magnitude in different bands stem from different locations in the disk. As demonstrated in $\S ?$ ?, the same apply for polarimetric features studied in this paper, which probes different disk regions. We can then track the variations of the local density at different locations in the disk. This fact therefore provides us with an interesting possibility of following viscous processes in $\mathrm{Be}$ disks such as dissipation, build-up or outburst phases. Particularly, it is useful to look at the correlation diagram of polarimetric observables at two different wavelengths.

Correlation features between $P_{B D}$ and $P_{V}$ were detected in Be stars that underwent build-up and dissipation phases (?). Those features form a clockwise loop and are well reproduced by VDD models. An example of these VDD predictions is presented in Figure ??. We can generalize the concept of this diagram by plotting other combinations of polarimetric observables that stem from different loci in the disk. In analogy to color magnitude diagrams (e.g. figure 22 of Paper I), this kind of diagram allows us to follow the evolution of the Be star inner and bulk disk. We hereafter name this kind of diagram polarization color loops (PCL).

The loop can be described as follows. When the mass injection starts, $P_{B D}$ increases from zero faster than $P_{V}$ until it reaches a limit value as seen on Figure ??. At the end of this first phase, the star has generated a large and dense CS disk. When mass injection stops, the inner disk quickly reaccretes back onto the star ; this causes a fast drop of $P_{B D}$. However, as described in the previous section, $P_{V}$ increases a bit more until accretion eventually consumes a significant part of the bulk disk and makes $P_{V}$ drop. The curve then follows a track towards the bottom-left part of the diagram. What follows is a slow secular dissipation of the entire disk along which $P_{B D}$ changes little (the inner disk having already reached very low densities) but $P_{V}$ diminishes as the disk mass decreases. The detailed shape of the loop depends on several factors as shown in Figure ??: the viewing angle and the value of $\alpha$.

To generate a PCL diagram, we need to plot a quantity that stems from the inner part of the disk as a function of a quantity that is produced over a larger extent in the disk. As an example, let's consider optical (UBVRI) polarimetric measurements. The $B$-band polarization being produced closer to the star than at longer wavelength, e.g. in the $I$ band, it is then possible to study viscous processes by monitoring the variation of $\frac{P_{B}}{P_{I}}$ as a function of $P_{V}$. All the diagrams made according to that principle contain roughly the same information in the sense that they enable to track observe building-up and dissipation phases (Figure ??).

\subsubsection{Diagnostic potential}

Having described the two main diagnostics contained in the polarized spectrum (polarization level and color) and how these relate to the disk properties, we now explore the diagnostic potential of polarimetric observations of Be disks.

We already saw on Figure ?? that PCLs present different shape according to the inclination angle of the system. Moreover, PCLs are characteristic of the density at the base of the disk and can be used to infer this quantity via VDD modeling. Figure ?? shows the different forms the PCL can take depending on the density at the base of the disk.

The amplitude of a PCL is a clear signature of the base density. In the case of Figure ??, the PCL has a bigger extent for a higher density, whatever the inclination angle. If the disk 

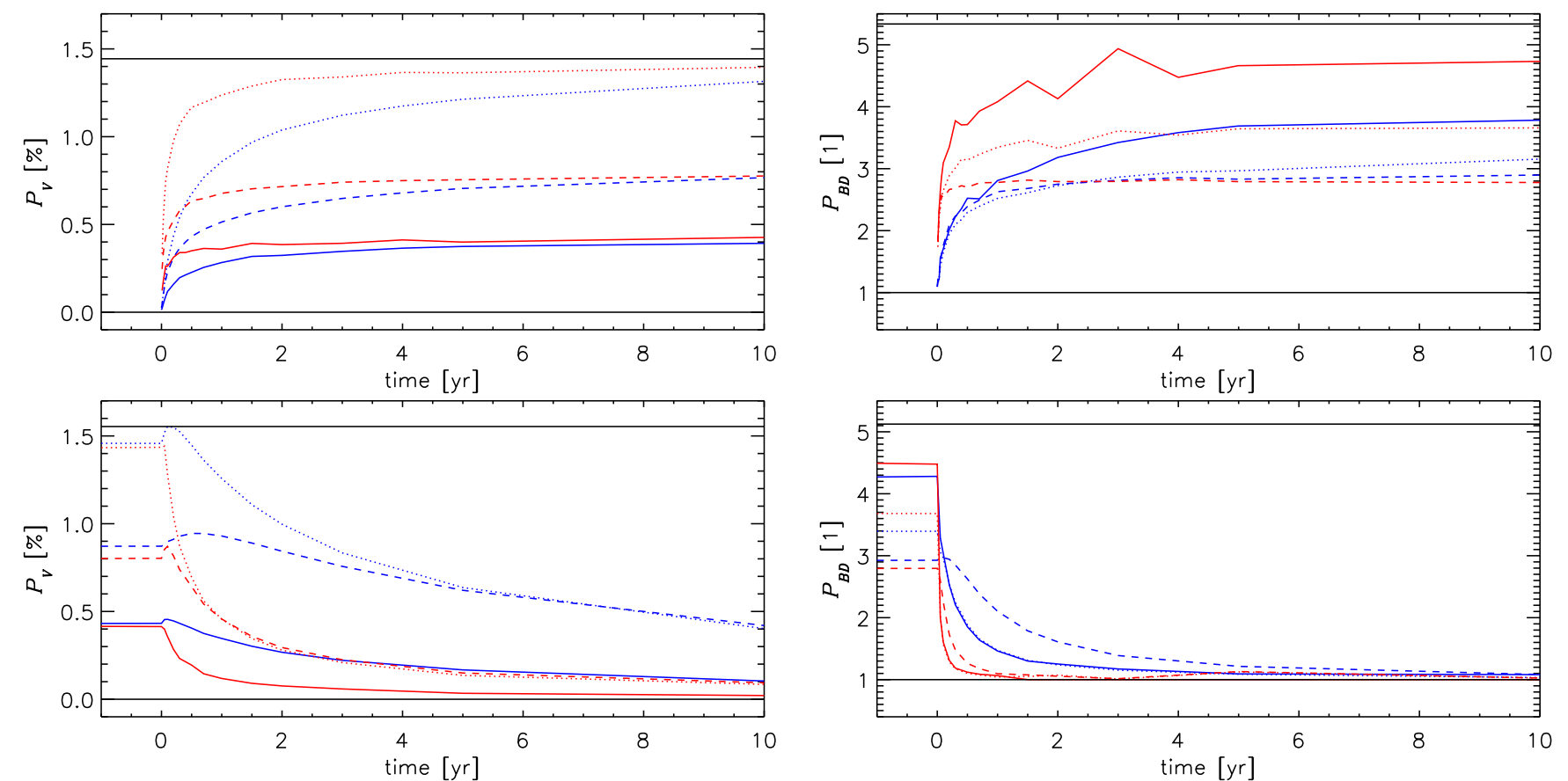

FIG. 5.- Temporal evolution of $P_{V}$ (left) and $P_{B D}$ (right) associated with disk build-up (top panel) and dissipation (bottom panel). The solid, dotted and dashed lines represent the lightcurves for inclination angles of $30^{\circ}$ (face-on), $70^{\circ}$ and $90^{\circ}$ (edge-on), respectively. The blue and red colors represent models for $\alpha=0.1$ and 1.0, respectively. The solid black lines indicate the maximum values for each scenario. 


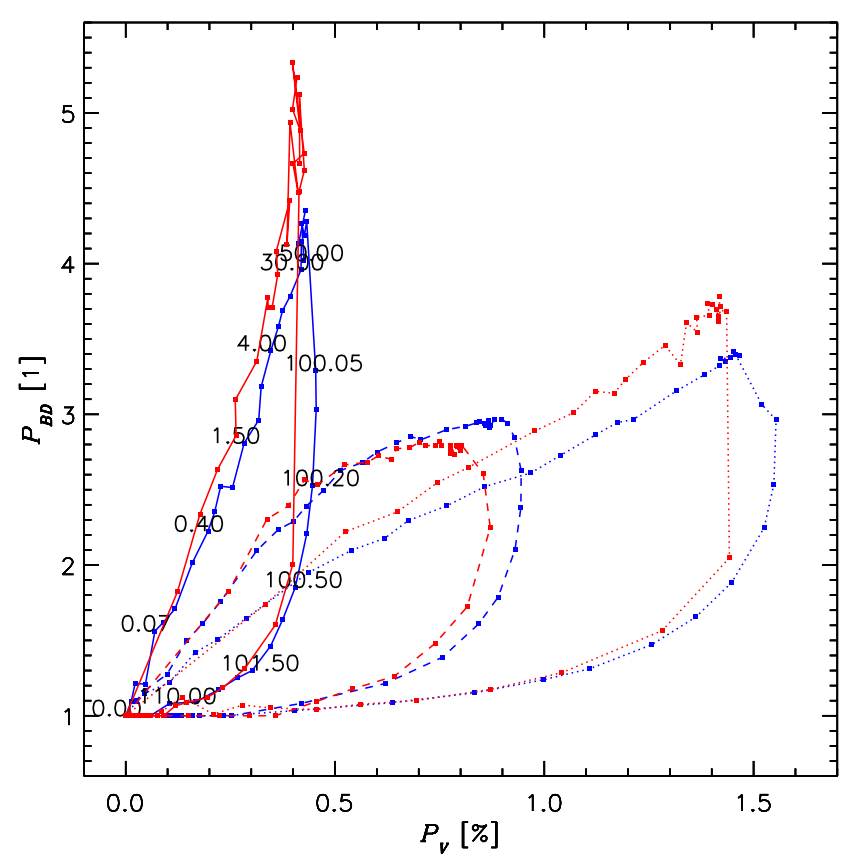

FIG. 6.- PCL $P_{B D}$ and $P_{V}$ at three inclination angles (solid line, 30 degrees, dotted line, 70 degrees and dashed line, 90 degrees) for a scenario involving a 100 year long building-up and 50 year dissipation and two $\alpha$ parameters (blue, 0.1 and red 1.0).
FIG. 7.- PCL showing $P_{B D}$ (dots), $P_{P D}$ (polarization at the Paschen jump, squares) and $\frac{P_{B}}{P_{I}}$ (triangles) as a function of $P_{V}$. The red curve shows a 50 year building up and the blue curve a following 50 year dissipation phase. Inclination angle is 90 degrees.

undergoes an almost constant building-up phase, the top region of the PCL is reached in less than a year (for a viscous coefficient of $\alpha=1$ ). Provided a few measurements typically spaced by one or two months and with $0.1 \%$ uncertainties, Figure ?? shows that it is quite straightforward to disentan-

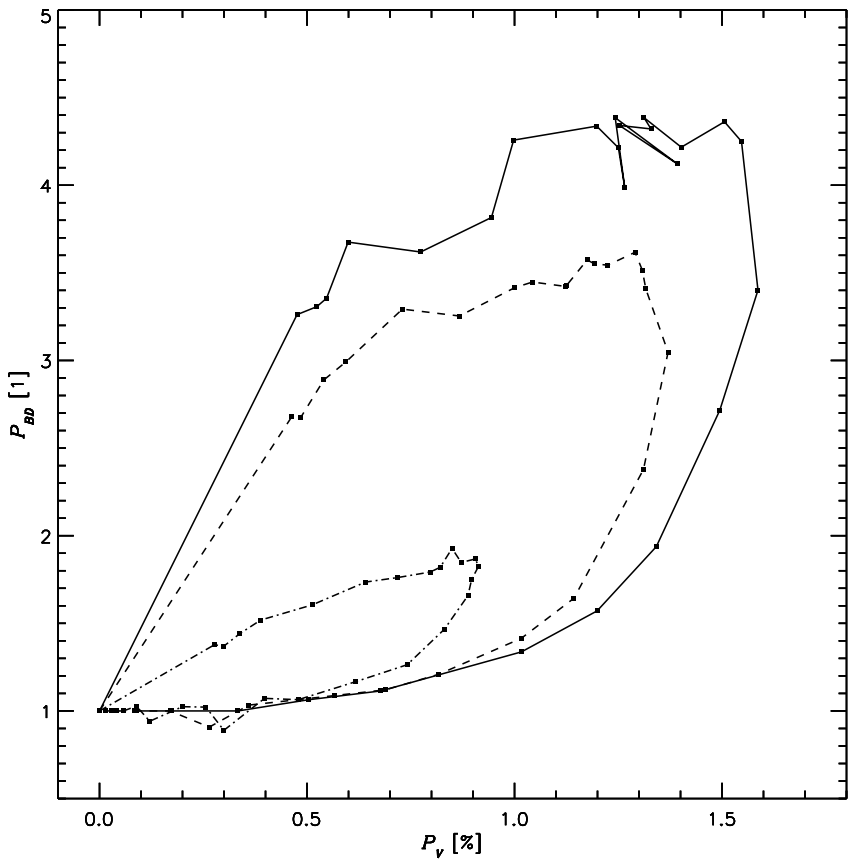

FIG. 8.- PCL of $P_{B D}$ and $P_{V}$ for 3 base densities: $6 \times 10^{-11} \mathrm{~g} \mathrm{~cm}^{-3}$ (solid line), $3 \times 10^{-11} \mathrm{~g} \mathrm{~cm}^{-3}$ (dashed line) and $1 \times 10^{-11} \mathrm{~g} \mathrm{~cm}^{-3}$ (dash-dotted line). The dynamical scenario involves a B3 star and 50 years of building-up followed by 50 years of dissipation. The inclination angle is 85 degrees.

gle between base densities that are different by a factor of 3 . We also note that the slope of the PCL changes with density. For low densities the slope is smaller and the loop less broad, while for large densities the slope increases and the loop gets broader. This is a result of the different weight that $\mathrm{H}$ I opacity plays in each model. Moreover, at very high densities $\left(\sim 1 \times 10^{-10} \mathrm{~g} \mathrm{~cm}^{-3}\right)$ and seen from the equator, PCL can exhibit an interesting secondary loop as plotted on Figure ??. The explanation for this phenomenon is based on absorption from the outer part of the disk. During the build-up phase, the disk gets so dense in its outer part that scattered photons from where $P_{B D}$ stem from are absorbed (point 1). When mass injection stops (point 2), both inner and outer parts of the disk dissipate. However the absorption from the outer part decreases faster than the number of scattering electrons in the inner part making the $P_{B D}$ level increase. On the other hand, $P_{V}$ increases because of a drop of the unpolarized flux then slowly decreases as the total disk mass vanishes (described in $\S ? ?)$. At point $3, P_{B D}$ drops due to the lack of density in the emptying inner part. This is why $P_{B D}$ cannot reach the values it had before absorption started and for that reason, the $P_{B D}$ level at the top of the secondary loop is always smaller than the level at the top of the primary loop. When the viscous disk has entirely been dissipated, $P_{B D}$ and $P_{V}$ eventually reach their final values of 1 and $0 \%$, respectively, as previously described in $\S$ ??. Since a low $\mathrm{T}_{\text {eff }}$ means a higher opacity, this effect is more important for late spectral type stars as shown on Figure ??.

In Figure ?? we saw how the maximum polarization depends on $\mathrm{T}_{\text {eff }}$. A strong dependence of the PCL on the central star spectral type is thus to be expected. On Figure ??, we can see that the spectral type impacts the PCL in various ways. Firstly, the range of $P_{V}$ values plotted in the PCL increases with increasing $\mathrm{T}_{\text {eff }}$ (as we already saw on Figure ??). Secondly, the slope of the PCL increases with decreasing $\mathrm{T}_{\mathrm{eff}}$. 


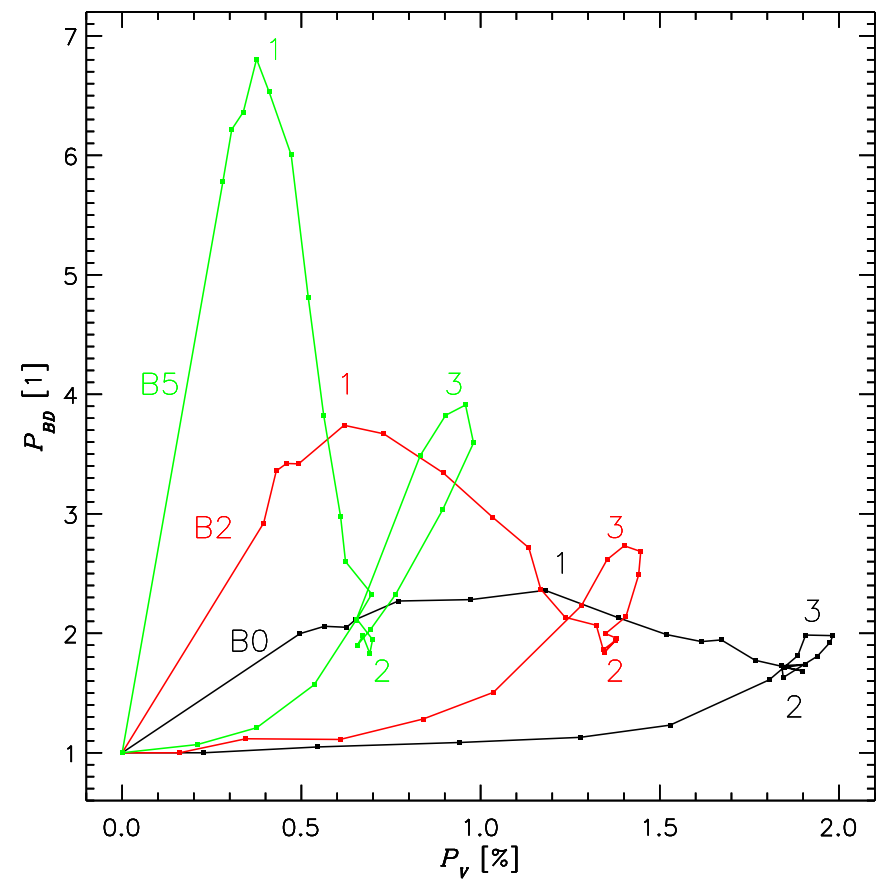

FIG. 9.- PCL of $P_{B D}$ and $P_{V}$ for a central star with 3 different spectral types. The base density is $1 \times 10^{-10}$ g. $\mathrm{cm}^{-3}$. The inclination angle is 90 degrees. Numbers indicate the three main phases of each scenario.

This again can be understood in terms of the relative importance of electron scattering opacity vs. H I opacity.

Indeed, the effective temperature has a strong effect on the opacity profile along the disk. For hotter objects, the opacity is quite low at the Balmer discontinuity. The $P_{B D}$ level is then rather low UNTRUE, to be modified, $P_{B D}$ level should be bigger. On the other hand, $P_{V}$ (that stems from the total extent of the disk) reaches high values because the rather low total opacity allows the photons to be scattered on a large volume throughout the disk (the correlation between the V-band polarization and effective temperature was already shown on Figure ??). The same principles apply for colder objects and bigger opacities. It will result in a PCL with a high amplitude in $P_{B D}$ and a smaller one in $P_{V}$. We conclude that the same kind of signature in the PCL is caused by either lowering the Teff (late spectral type) or increasing the overall density. Therefore, we conclude that the real diagnostic potential of the PCL depends critically on good estimates of the spectral type of the central star (e.g., using spectroscopic measurements).

In the work presented in ?, the simulation of the growth and dissipation phases are artificially reproduced by changing the size of the outer radius and inner radius of the disk, respectively. The disk density is assumed to stay in the ideal quasi steady-state regime with a power-law index of $n=3.5$ and the same base densities. With this approach, they corroborate the mechanism provided in ? to explain observed PCLs for $\pi$ Aqr and 60 Cyg (see \$??. One important difference with the here-presented results lies in the shape of the PCL for different spectral types. While Halonen et al. find the $P_{B D}$ maximum levels increase for hotter stars, we showed the opposite behavior on Figure ??. CHECKING: the opacity is lower for earlier ST at 0.364 microns (from the analytical part, figure 2), why do decretion models show a lower $P_{B D}$ then ? .

\subsection{Periodic mass loss rate}

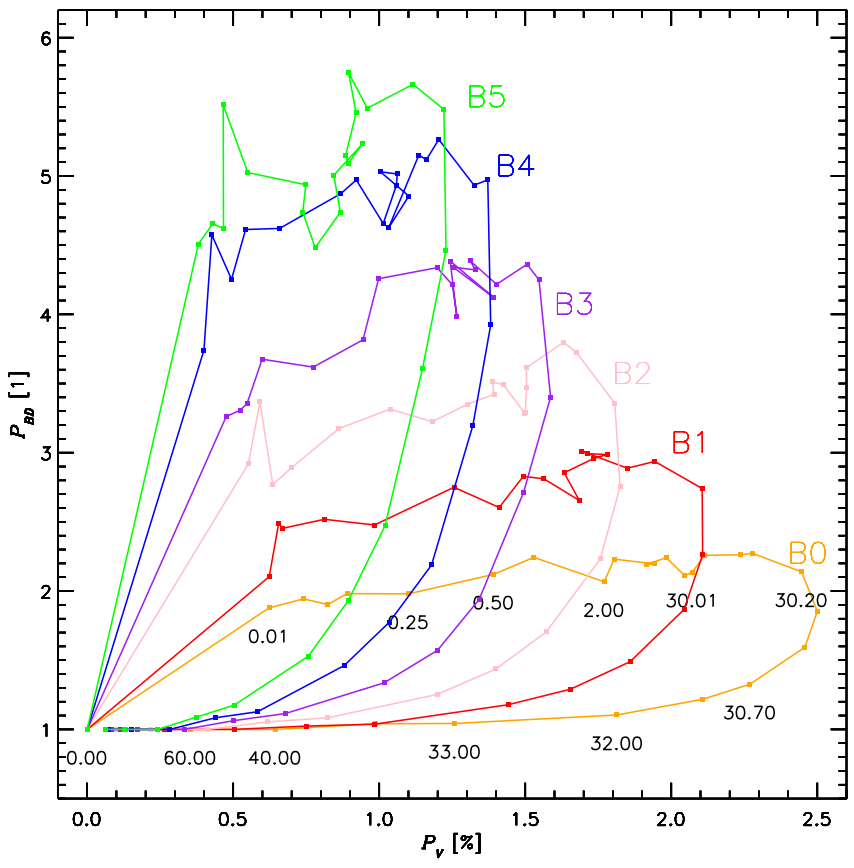

FIG. 10. - PCL of $P_{B D}$ and $P_{V}$ for a central star of different spectral types. The base density is $6 \times 10^{-11}$ g.cm $\mathrm{cm}^{-3}$. The inclination angle is 85 degrees.

Periodic activity from Be stars has been widely observed with photometric measurements. (e.g. ?). It is thus important to investigate how VDD models can reproduce this variability and to know what kind of signatures one can expect from polarimetric observables.
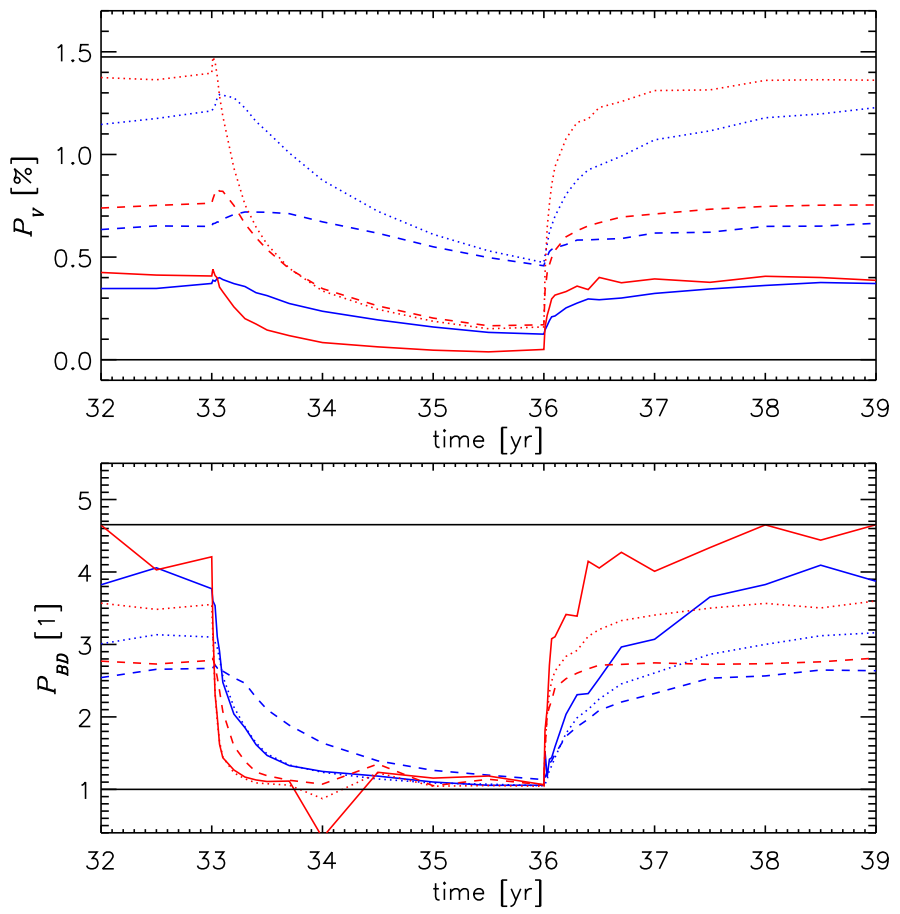

FIG. 11. - Same as for Figure ?? for a periodic mass injection scenario with period of 6 years and a duty cycle of $50 \%$.

Figure ?? shows the temporal evolution of $P_{V}$ and $P_{B D}$ for a periodic mass injection scenario. This scenario involves a 
periodic injection rate with a period of 6 years and a duty cycle of $50 \%$ (meaning that the stellar mass is continuously injected into the disk for three years every six years). Logically, the resulting polarization curves are a succession of buildingup and dissipation curves. Unlike the scenarios seen in $\S ? ?$ ? the disk doesn't totally build and dissipate. The lowest values of $P_{V}$ and $P_{B D}$ at the end of quiescence are therefore different from $0 \%$ and 1, respectively, and the maximum values are less than the ones we would have for an uninterrupted building-up phase (Figure ??).

For sufficiently long phases of building-up and dissipation, the disk reaches or almost reaches a quasi-steady state. In a PCL diagram, this translates into a closed loop with a maximal extent. However, for scenarios where the mass injection is periodically variable, the PCL also shows a loop-shape but with a lower amplitude, simply because the interruption of the mass injection stops the observables from reaching their maximum values. Another feature of periodic scenarios is that the loop might evolve from cycle to cycle. This depends on the parameters of the scenario and is visible mainly for low period scenarios (i.e. about a year or less for $\alpha=1.0$ ). Since the disk builds faster than it vanishes (as seen with polarimetry both in $V$-band and at the Balmer discontinuity on Figure ??), the disk doesn't have the time to dissipate all the matter that was injected during the build-up phase of a given $n$ cycle. Consequently, the $P_{V}$ (and in a lesser extent $P_{B D}$ ) value at the beginning of the $n+1$ cycle will be higher than at the beginning of the $n$ cycle. As a result, the rest of the $n+1$ loop will also look different than the previous ones. This is illustrated on Figure ??. We can see that the loop representing a variable mass injection scenario is confined inside the loop corresponding to a fully-developed/dissipated disk. This demonstrates the diagnosis potential of this type of plot to infer periodic scenarios in Be stars.

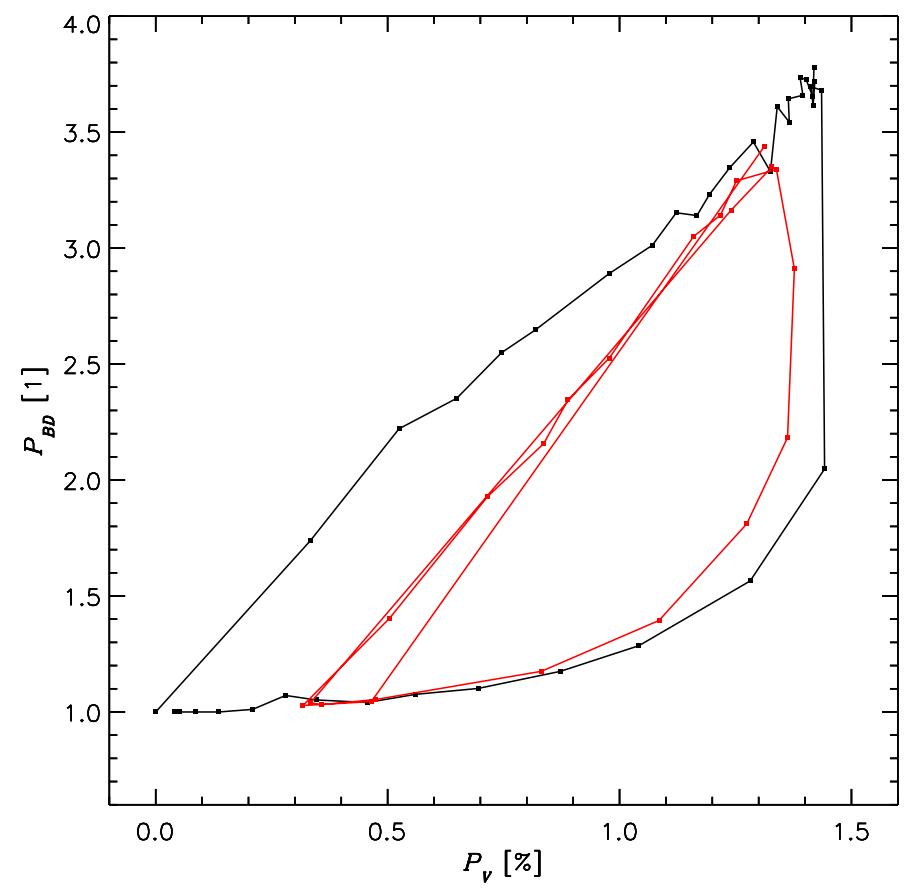

FIG. 12.- PCL of $P_{B D}$ and $P_{V}$ at 70 degrees of inclination angle for the same dynamical scenario shown in Figure ?? (in black) and for another one where the mass injection rate is periodically turned on and off every one year (in red).
With a limited time coverage of observed measurements, it is very likely that the here-presented synthetic polarization curves will not be constrained enough and a same dataset could equally be modeled by different dynamical scenarios and star/disk parameters. The solution remains in the observation at more wavelengths to break this degeneracy.

\subsection{Episodic mass loss rate}

In the dynamical scenarios previously shown, the diagrams present loop shapes because the density that is injected at the base of the disk is constant. However, this would not be the case if the mass injection rate was to vary as it can be expected during outburst events for example.

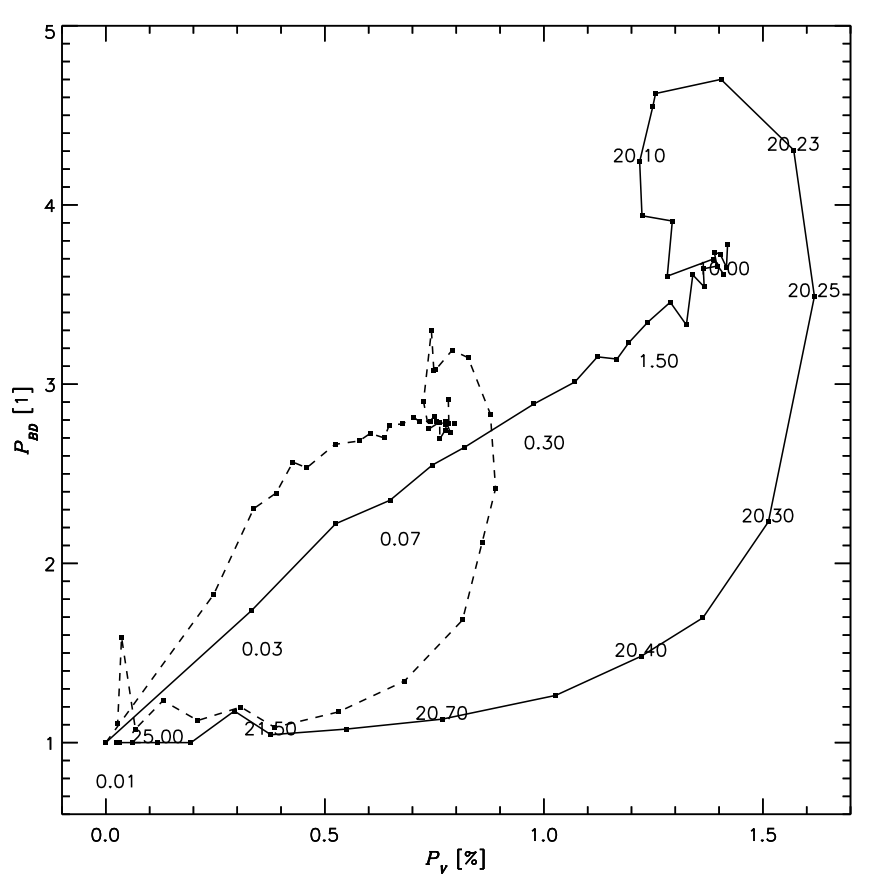

FIG. 13.- PCL of $P_{B D}$ and $P_{V}$ showing a 0.2 year outburst after a 20 yearlong stable period of building-up. The outburst is then followed by a 30 year dissipation. The solid and dashed lines represent inclination angles of 70 and 90 degrees, respectively.

Figure ?? represents a PCL where the dynamical scenario involves a 20 -year long building-up phase followed by a 0.2 year outburst with a mass injection rate twice higher than during the build-up. The outburst is then followed by a 30 year dissipation phase. After the outburst happened at $\mathrm{t}=20$ years, $P_{B D}$ brutally increased while $P_{V}$ stays essentially the same for the first 0.1 year after the outburst has started. When the outburst stops, $P_{B D}$ immediately falls down while $P_{V}$ increases as the outer disk fills up. The two quantities eventually decrease to $0 \%$ and 1 as the whole disk dissipates. The outburst thus adds an extra outgrowth to the loop compared to a dynamical scenario without any outburst and should be quite wellidentified provided a sufficient time-coverage of polarimetric observations.

\section{DISCUSSION/COMPARISON OF THE PREDICTIONS WITH OBSERVED DATA}

Long-term polarimetric observations of the Be stars $60 \mathrm{Cyg}$ and $\pi$ Aqr were obtained with the spectropolarimeter HPOL and revealed several year long disk-loss episodes interrupted by polarimetric outbursts (?). $P_{B D}-P_{V}$ PCLs made out of 
these measurement showed loop patterns whose general shape was successfully described with viscous disk build-up and dissipation phases (?) similar to the ones we detail in the present paper.

Thanks to the theoretical work we developed in the previous sections, we are now able to better understand the details of these observed PCLs from 60 Cyg and $\pi$ Aqr (Figure 1 of ?).

PUT the figures AGAIN, ask ZACH!

$$
\text { 4.1. } \pi A q r
$$

$\pi$ Aqr PCL presents a very clear observational evidence of a partial loop obtained during a $\sim 180$ day polarimetric outburst. From a pre-existent disk $\left(P_{V}=0.9 \%, P_{B D}=1.8\right)$, the loop starts with a build-up phase until it reaches a maximum $P_{B D}$ value of 2.3. Mass injection stops in the inner disk $\left(P_{B D}\right.$ drops) and the rest of the disk progressively disappears under the effect of viscosity. Nonetheless, an increase in $P_{B D}$ is visible during this dissipation phase. Since $P_{V}$ doesn't increase in turn, a second building-up phase is discarded. It might be representative of a small injection of matter in the inner disk that doesn't efficiently affect a bigger extent of the disk during the duration of the outburst.

As already reported in ? and in the present paper, the general clockwise loop is well explained by viscous processes. The exact PCL then depends on an interplay between the injected quantity of matter, the inclination angle, the $\alpha$ parameter and a good knowledge of the disk state before the outburst. Such in depth analysis will be carried out in the future. However, we can say that $\pi$ Aqr being a $B 1$ type star (?), the range of the observed $P_{V}$ and $P_{B D}$ values are compatible with our VDD predictions as shown in Figure ??.

\section{2. $60 \mathrm{Cyg}$}

60 Cyg presents a more complex case. Instead of showing a well-defined clockwise loop, this PCL exhibits uneven structures that result from a serie of outbursts from the central star. The slopes are quite high which, for a $B 1$ spectral type (?), are characteristic of high base densities and/or low inclination angles.

The first points of the PCL can represent a build-up phase until the top of the loop. Then $P_{B D}$ drops suggesting an interruption of the mass supply in the disk until a value of $P_{B D} \sim 1$, which means that its inner part is basically empty. This first clockwise part of the PCL is well understood as a classical building-up/dissipation scenario. However, another mass injection into the disk makes $P_{B D}$ level rise again while $P_{V}$ values continue to decrease with the dissipation of the bulk disk. This behaviour remains quite enigmatic since, similarly to $\pi$ Aqr, no increase in $P_{V}$ is seen. This might also be a signature of a decaying strong absorption from the outer disk seen at a high inclination angle. Another explanation lies in the fact that 60 Cyg is known to be a spectroscopic binary with a 146 day orbital period (?). A gravitational interaction of the disk with the companion could lead to a truncation of the outer reaches and stops the $P_{V}$ to fully develop. Finally, at a point where this second counter-clockwise part connects with the first clockwise part, the $P_{B D}$ level drops together with $P_{V}$. After this last stage, the inner disk has entirely vanished whereas a high enough density remains further away from the star since the $P_{V}$ level stabilizes around $0.5 \%$.

$$
\text { 4.3. } \Psi \mathrm{Per}
$$

Another observation case that we can compare to our models, especially with Figure 8, is $\psi$ Per (B5; inclination $=75$
+/-8 (Delaa et al. 2011)) which exhibits a partial PCL loop ed by a huge jump then decrease in the BJ ratio, with only minimal change in the V-band polz (Figure ??). We refer the reader to Draper et al (2013) for further details regarding the observational data. Zack - can you send out that figure, along with a brief explanation of the 'blue' data points? (you might need to tweak the y-axis of the PCL plot, as it looks like 1 data point is off-the-graph)

As for Psi Per, here is a zoom in of the loop, the data point which is off scale is 80 , if I scale it to that its hard to see the rest of the loop (effectively BJ minus filter is zero). It approaches steady-state with a decreasing BJ, and has maybe a slight mini-loop before having disk loss. Is there an inclination limit for this loop behaviour? Perhaps the actual inclination should be closer to 90 then determined by interferometry.

I've attached a figure of psi Per and show a blow up of the second loop. Its building up as it descends from above in the PCL, is in steady state for a few observations and then goes counter clock wise in a rapid 189 days, which was unfortunately not as well time sampled. Let me know what you think to update the figure or add caption/discription to it for the paper.

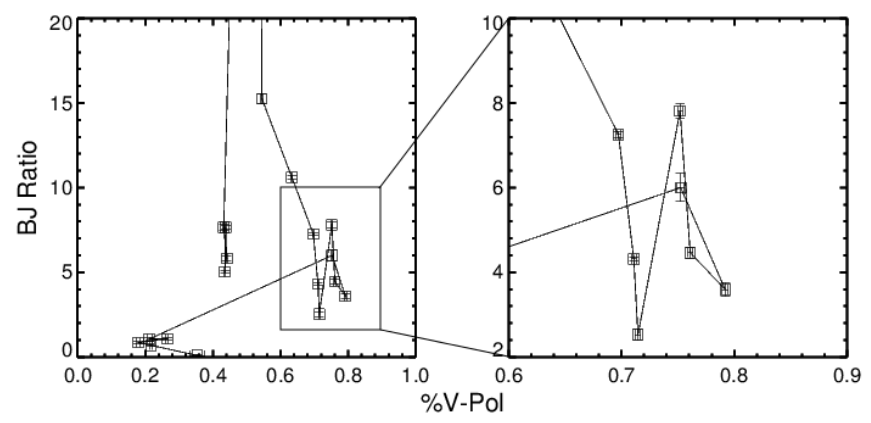

Fig. 14.-

It is important to mention that PCLs can also be qualitatively reproduced using spiral oscillation models as demonstrated in the Figure 9 of ?. In this approach, a one armed density wave that is confined to a region extending out to $10 R_{\star}$ generate distinct shapes for the PCLs as well as characteristic signatures in the polarization angle variation. We conclude by saying that a detailed modeling specific to each observed Be star is needed to understand the origin of the polarimetric variability. PCLs and polarization angle measurements are critical observable quantities to disentangle a scenario involving a variable mass injection rate in an axisymmetric disk (with no polarization angle variation expected) from a scenario including a one-armed oscillation.

\section{CONCLUSIONS}

In this paper, we first reviewed the mechanisms at the origin of the polarization levels observed from Be stars under the assumption of a steady state VDD model. With a more realistic modeling based on a coupling of hydrodynamics and radiative transfer simulations, we showed how some polarimetric observables evolve with time for different mass injection scenarios. We saw that the mass injection history (constant, periodic or episodic) results in specific behavior of the polarimetric observables. This led us to introduce a new diagnostic tool that we named PCL (Polarization Color Loop) as an analogy to color magnitude diagrams. We then showed that PCLs 
constitute a powerful diagnosis tool to derive different physical parameters of the disk such as the inclination angle, the viscous coefficient $\alpha$, the disk base density and the spectral type of the central star on top of the mass injection history. We mention it is possible to derive the inclination angle and the spectral type independently to limit or verify those free parameters when interpreting a PCL. Based on these considerations, we eventually commented some observed PCLs with the study of $\pi$ Aqr, 60 Cyg and $\Psi$ Per cases. From the figures we plotted, we also showed there is some degenerescence, i.e. several sets of mass injection scenarios and parameters could equally reproduce a same observed PCL. And consequently we stress that the more observables, the easier to infer a dynamical scenario and physical parameters of the system.

$\mathrm{XH}$ wants to thanks FAPESP for supporting this work through the grants 2009/07477-1 and 2010/19029-0. Alex, Bruno? This work has made use of the computing facilities of the Laboratory of Astroinformatics (IAG/USP, NAT/Unicsul), whose purchase was made possible by the Brazilian agency FAPESP (grant 2009/54006-4) and the INCT-A.

\section{REFERENCES}

Bjorkman, J. E., \& Bjorkman, K. S. 1994, ApJ, 436, 818

Bjorkman, J. E. 1997, Stellar Atmospheres: Theory and Observations, 497, 239

Bjorkman, J. E., \& Carciofi, A. C. 2005, The Nature and Evolution of Disks Around Hot Stars, 337, 75

Carciofi, A.C. \& Bjorkman, J.E. 2006, ApJ, 639, 1081

Carciofi, A. C., \& Bjorkman, J. E. 2008, ApJ, 684, 1374

Carciofi, A. C., Magalhães, A. M., Leister, N. V., Bjorkman, J. E., \& Levenhagen, R. S. 2007, ApJ, 671, L49

Carciofi, A. C., Okazaki, A. T., Le Bouquin, J.-B., Svtefl, S., Rivinius, T., Baade, D., Bjorkman, J. E., \& Hummel, C. A. 2009, A\&A, 504, 915

Carciofi, A. C. 2011, IAU Symposium, 272, 325

Carciofi, A. C., Bjorkman, J. E., Otero, S. A., et al. 2012, ApJ, 744, L15

Draper, Z. H., Wisniewski, J. P., Bjorkman, K. S., Haubois, X., Carciofi, A. C., Bjorkman, J. E., Meade, M. R., \& Okazaki, A. 2011, ApJ, 728, L40

Halonen, R. J., Mackay, F. E., \& Jones, C. E. 2013, ApJS, 204, 11

Halonen, R. J., \& Jones, C. E. 2013, ApJ, 765, 17

Halonen, R. J., \& Jones, C. E. 2013, arXiv:1307.6220

Haubois, X., Carciofi, A. C., Rivinius, T., Okazaki, A. T., \& Bjorkman, J. E. 2012, ApJ, 756, 156

Jones, C. E., Sigut, T. A. A., \& Porter, J. M. 2008, MNRAS, 386, 1922

Koubský, P., Harmanec, P., Hubert, A. M., et al. 2000, A\&A, 356, 913

Lee, U., Osaki, Y., \& Saio, H. 1991, MNRAS, 250, 432
Meilland, A., Millour, F., Kanaan, S., et al. 2012, A\&A, 538, A110

Okazaki, A. T. 2001, PASJ, 53, 119

Okazaki, A. T. 2007, Active OB-Stars: Laboratories for Stellare and Circumstellar Physics, 361, 230

Porter, J. M., \& Rivinius, T. 2003, PASP, 115, 1153

Porter, J. M. 1999, A\&A, 348, 512

Pringle, J.E. 1981, ARAA, 19, 137

Quirrenbach, A., Bjorkman, K. S., Bjorkman, J. E., et al. 1997, ApJ, 479, 477

Sabogal, B. E., Mennickent, R. E., Pietrzyński, G., et al. 2008, A\&A, 478, 659

Shakura, N. I., \& Sunyaev, R. A. 1973, A\&A, 24, 337

Sigut, T. A. A., \& Jones, C. E. 2007, ApJ, 668, 481

Slettebak, A. 1982, ApJS, 50, 55

Tycner, C., Jones, C. E., Sigut, T. A. A., Schmitt, H. R., Benson, J. A., Hutter, D. J., \& Zavala, R. T. 2008, ApJ, 689, 461

Wisniewski, J. P., Draper, Z. H., Bjorkman, K. S., et al. 2010, ApJ, 709, 1306 Wheelwright, H. E., Bjorkman, J. E., Oudmaijer, R. D., et al. 2012, MNRAS, 423, L11

Wood, K., Bjorkman, J. E.,Whitney, B., \& Code, A. 1996, ApJ, 461, 847

Wood, K., Bjorkman, K. S., \& Bjorkman, J. E. 1997, ApJ, 477, 926

de Wit, W. J., Lamers, H. J. G. L. M., Marquette, J. B., \& Beaulieu, J. P. 2006, A\&A, 456, 1027 